\title{
Ökonometrische Verfahren zur Messung von Segregation und Lohndiskriminierung - eine theoretische und empirische Studie
}

\author{
Dissertation \\ zur Erlangung des Doctor rerum politicarum der Wirtschaftswissenschaftlichen Fakultät \\ der Universität Göttingen
}

vorgelegt von Carsten Hundertmark aus Rehren A/O, Deutschland

Göttingen, 2012 
Erstgutachter: Prof. Dr. Stefan Sperlich

Zweitgutachter: Prof. Dr. Thomas Kneib

Tag der mündlichen Prüfung: 2. Mai 2012 


\section{Danksagung}

Ich möchte mich bei meinem Betreuer, Herrn Prof. Dr. Stefan Sperlich, für seine Ideen und Anregungen sowie seine akademische Unterstützung bedanken. Desweiteren möchte ich mich bei Herrn Prof. Dr. Kneib und Frau Professor Dr. Martínez-Zarzozo für Ihre Bereitschaft, als Zweit- und Drittgutachter zu agieren, bedanken.

Ein weiterer Dank für ihre Unterstützung geht an meine Kollegen am Institut für Statistik und Ökonometrie der Georg-August Universität Göttingen. Besonders hervorheben möchte ich dabei Herrn Dr. Oleg Nenadić, der mir bei sämtlichen Fragen zur Programmierung insbesondere bei den R-Paketen weiterhalf.

Ich danke Frau Silke Staack für das Korrekturlesen meiner Dissertation sowie meiner Familie für ihre Unterstützung und Ihren Glauben an mich. 


\section{Inhaltsverzeichnis}

\begin{tabular}{lll}
\hline 1 & Einführung & 1
\end{tabular}

2 Methoden zur Messung von Segregation 3

2.1 Einleitung . . . . . . . . . . . . . . . . . . . . . . . . 3

2.2 Traditionelle Messverfahren . . . . . . . . . . . . . . . . . . . . . 5

2.2 .1 Methodologie . . . . . . . . . . . . . . . 5

2.2 .2 Empirische Resultate . . . . . . . . . . . . . . . . . . . . 8

2.3 Auf Entropie basierende Verfahren . . . . . . . . . . . . . . . . . . . 10

2.3.1 Der Informationstheorieindex nach Theil (1972 . . . . . . . . . . . . 10

2.3.2 Der Mutual-Information-Index nach Frankel und Volij (2008) . . . . 11

$2.3 .3 \quad$ Empirische Resultate . . . . . . . . . . . . . . . . . . . . 15

2.4 Der Gini-Koeffizient nach Kim und Jargowsky (2005) . . . . . . . . . . . . 16

2.4 .1 Methodologie . . . . . . . . . . . . . . . 16

2.4 .2 Empirische Resultate . . . . . . . . . . . . . . . . . . . . . . . . . 19

2.5 Messung ordinaler Segregation nach Reardon (2008) . . . . . . . . . . . . . 20

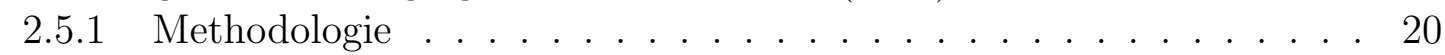

2.5 .2 Empirische Resultate . . . . . . . . . . . . . . . . . . . . . . . . . . . . . . . . . . . . . 25

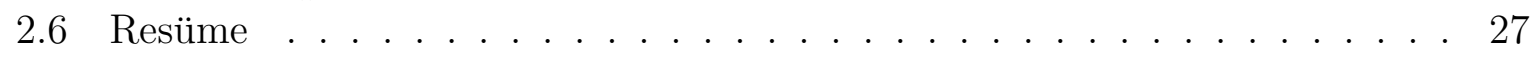

\begin{tabular}{|lll}
3 & Methoden zur Messung von Lohndiskriminierung & 31
\end{tabular}

3.1 Einleitung . . . . . . . . . . . . . . . . . . . . . . 31

3.2 Die Daten $\ldots \ldots \ldots \ldots$. . . . . . . . . . . . . . . . . . . . . . . . . 34

3.3 Auf Lohnregressionen basierende Methoden . . . . . . . . . . . . . . . . 36

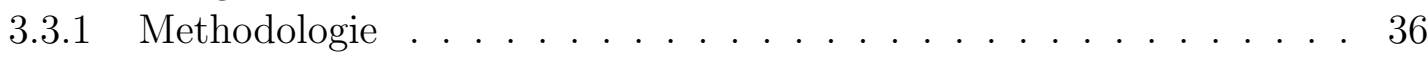

3.3 .2 Empirische Resultate . . . . . . . . . . . . . . . . . . . . . . . . 38

$3.4 \quad$ Berufliche Segregation berücksichtigende Verfahren . . . . . . . . . . . . . 41

3.4.1 Dekomposition nach Brown, Moon und Zoloth (1980)] . . . . . . . . 41

3.4.2 Dekomposition nach Appleton, Hoddinott und Krishnan (1999) . . 43

3.4.3 Empirische Resultate . . . . . . . . . . . . . . . . . . . 45

3.5 Methoden auf Basis kontrafaktischer Verteilungen . . . . . . . . . . . . . . 48

3.5.1 Dekomposition nach DiNardo, Fortin und Lemieux (1996) . . . . . 49

3.5.2 Dekomposition nach Machado und Mata (2005), Melly (2005) . . . 53

3.6 Auf Matching basierende Dekomposition nach Nopo (2008) . . . . . . . . . 56 
3.6 .1 Methodologie . . . . . . . . . . . . . . . 56

3.6 .2 Empirische Resultate . . . . . . . . . . . . . . . . . . . . . . . . . 60

3.7 Diskriminierung im Zeitverlauf . . . . . . . . . . . . . . . . . . . . . . . . . 61

3.7 .1 Methodologie . . . . . . . . . . . . . . . . . 61

3.7 .2 Empirische Resultate . . . . . . . . . . . . . . . . . . . . 63

3.8 Resüme $\ldots \ldots \ldots \ldots \ldots \ldots$. . . . . . . . . . . . . . . . . . . . . . . . . . 65 


\section{Abbildungsverzeichnis}

2.1 Lorenzkurven für individuelle Haushalte und Regionen . . . . . . . . . . . 16

2.2 Kumuliertes Haushaltseinkommen . . . . . . . . . . . . . . . . . . . . . . . 18

3.1 Dichtefunktionen der logarithmierten Bruttostundenlöhne. . . . . . . . . . 52

3.2 Dichtefunktionen der logarithmierten Bruttostundenlöhne . . . . . . . . . . 55 


\section{Tabellenverzeichnis}

2.1 Aufteilung von Frauen und Männern auf unterschiedliche Berufsgruppen . 9

$2.2 \quad$ Ergebnisse der Segregationsmessungen nach traditionellen Methoden . . . . 9

2.3 Ergebnisse der Segregationsmessungen nach Theil (1972), Frankel und Volij

2.4 Aufteilung von Haushalten auf die Regionen . . . . . . . . . . . . . . . 20

2.5 Ergebnisse der Segregationsmessungen nach Kim und Jargowsky (2005) . . 20

2.6 Unterschiedliche Szenarien der Berufsaufteilungen . . . . . . . . . . . . 21

2.7 Aufteilung von Inländern, -innen und Ausländern, -innen auf die Berufs-

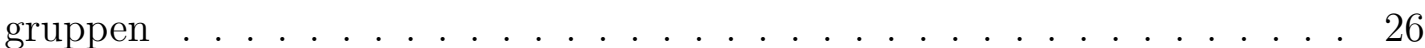

2.8 Ergebnisse der Segregationsmessungen nach Reardon (2006) bei unterschiedlich gewählten ungeordneten Kategorien . . . . . . . . . . . . . . . . . . . 26

3.1 Zusammenfassung der Gewichtungsmatritzen . . . . . . . . . . . . . . 38

3.2 Koeffizientenschätzung für die Stichprobe der Männer (2006) . . . . . . . . 39

3.3 Koeffizientenschätzung für die Stichprobe der Frauen (2006) . . . . . . . . . 39

$3.4 \quad$ Ergebnisse der Zerlegung nach OB, Neumark (1988) (a) . . . . . . . . . . . 40

$3.5 \quad$ Ergebnisse der Zerlegung nach OB, Neumark (1988) (b) . . . . . . . . . . . . 41

3.6 Koeffizientenschätzung der Ordinal-Regression für die Stichprobe der Männer 46

3.7 Aufteilung von Frauen und Männern auf die Berufsgruppen . . . . . . . . . 47

3.8 Ergebnisse der Dekomposition nach Brown, Moon und Zoloth (1980), Appleton, Hoddinott und Krishnan (1999) $\ldots \ldots \ldots$. . . . . . . . . . 48

3.9 Koeffizientenschätzung des Logitmodells der gepoolten Stichprobe . . . . . 51

3.10 Ergebnisse der Dekomposition nach DiNardo, Fortin und Lemieux (1996) . 51

3.11 Ergebnisse der Dekomposition nach Machado und Mata (2005), Melly (2005) 56

3.12 Ergebnisse der Dekomposition nach Nopo (2006) $\ldots \ldots$. . . . . . . . 60

3.13 Entwicklung der Reallöhne im Zeitraum von 1985-1995 . . . . . . . . . . . 64

3.14 Koeffizientenschätzung für die Stichprobe der Männer 1985 . . . . . . . . . . 64

3.15 Koeffizientenschätzung für die Stichprobe der Männer 1995 . . . . . . . . . 65

3.16 Ergebnisse der Dekomposition nach Juhn, Murphy und Pierce (1991) . . . 65 


\section{Kapitel 1}

\section{Einführung}

Im Rahmen dieser Dissertation werden ökonometrische Verfahren zur Messung von Segregation und Lohndiskriminierung vorgestellt. Segregation ist ein viel diskutiertes soziales Thema. Berufliche Segregation liegt vor, wenn sich unterschiedliche Gruppen ungleich auf einzelne Berufe oder Berufsgruppen aufteilen. Üben beispielsweise Frauen in größerem Maße schlechter bezahlte Berufe aus als Männer, so kann dies eine Erklärung für die Lohnlücke zwischen weiblichen und männlichen Arbeitnehmern sein. Regionale Einkommenssegregation liegt vor, wenn sich verschiedene ethnische Gruppen ungleich auf einzelne Regionen aufteilen. Segregation in Schulen kann zu Unterschieden im Bildungsniveau zwischen Schülern aus unterschiedlichen ethnischen Gruppen beitragen. In Kapitel 2 werden die gängigen Methoden zur Messung von Segregation vorgestellt, verglichen sowie Stärken und Schwächen der einzelnen Verfahren diskutiert. Die methodischen Ansätze stehen hierbei im Vordergrund, aber auch die Darstellung empirischer Ergebnisse ist Gegenstand der nachfolgenden Ausführungen.

In Deutschland liegt der durchschnittliche Lohn einer weiblichen Arbeitskraft unter dem einer männlichen Arbeitskraft. Diese Lohnunterschiede werden in politischen Debatten manchmal als Anzeichen dafür gewertet, dass viele Unternehmer Frauen diskriminieren. Allerdings ist zu beachten, dass selbst auf einem Arbeitsmarkt ohne Diskriminierung unterschiedliche Menschen Löhne in unterschiedlicher Höhe erhalten würden. Das alleinige Beobachten von Lohnunterschieden zwischen großen Gruppen erlaubt keine Aussagen über die Gewichtigkeit der Diskriminierung. Bei sämtlichen in Kapitel 3 vorgestellten Verfahren wird die Lohnlücke in einen durch unterschiedliche Humankapitalausstattungen erklärten Teil und einen unerklärten Teil, der als Maß für Diskriminierung interpretiert werden kann, zerlegt. Methodische Ansätze sind ebenso Gegenstand der nachfolgenden Ausführungen wie die Darstellung empirischer Ergebnisse, die sich auf den deutschen Arbeitsmarkt konzentrieren.

Im dritten Teil dieser Arbeit werden in der Open Source Software R für die in Kapitel 2 und 3 vorgestellten Verfahren zur Messung von Segregation und Lohndiskriminierung zugehörigen Pakete "segregation" und "discrimination" gebaut. 


\section{Kapitel 2}

\section{Ökonometrische Methoden zur Messung von Segregation}

\section{1 $\quad$ Einleitung}

Segregation ist ein viel diskutiertes soziales Thema. Berufliche Segregation liegt vor, wenn sich unterschiedliche Gruppen ungleich auf einzelne Berufe oder Berufsgruppen aufteilen. Üben beispielsweise Frauen in größerem Maße schlechter bezahlte Berufe aus als Männer, so kann dies eine Erklärung für die Lohnlücke zwischen weiblichen und männlichen Arbeitnehmern sein. Regionale Einkommenssegregation liegt vor, wenn sich verschiedene ethnische Gruppen ungleich auf einzelne Regionen aufteilen. Segregation in Schulen kann zu Unterschieden im Bildungsniveau zwischen Schülern aus unterschiedlichen ethnischen Gruppen beitragen.

Zur Messung von Segregation wurden in den letzten Jahren zahlreiche Indizes entwickelt. Der Dissimilaritätsindex ID geht auf Duncan und Duncan (1955) zurück ist auch heute noch der vor allem in der amerikanischen Literatur weit verbreitetste Index. Nach Falk (2002) wird der ID interpretiert als Prozentsatz der Frauen oder Männer, die ihren Beruf wechseln müssten, damit eine Gleichverteilung von Männern und Frauen entsprechend ihrer Verteilung in der Gesamtbeschäftigung vorliegt. Während der oben beschriebene Dissimilaritätsindex ID die Segregation entsprechend der Berufsgröße gewichtet, werden bei der Berechnung des standardisierten Dissimilaritätsindizes SID nach Gibbs (1965) und Gross (1968) alle Berufsgruppen gleich gewichtet. Der Informationstheorieindex $H$ (Theil (1972), Theil und Finezza (1971), Zoloth (1976)) misst, wie ungleich sich Männer und Frauen auf Berufe verteilen, wobei die ungleiche Verteilung von Arbeitskräften als Entropie definiert wird. Der Sex Ratio $S R$ wird von Hakim (1981) angewandt, um Segregation am Britischen Arbeitsmarkt zu analysieren. Hakim (1981) beschreibt dieses Maß als den Unterschied zwischen dem Ausmaß an Überpräsentation von weiblichen Arbeitskräften in typischen Frauenberufen und Unterpräsentation von weiblichen Arbeitskräften in typischen Männerberufen. Blackburn et al. (1995) entwickeln eine standardisierte Form dieses Indizes. James und Taeuber (1985) definieren den Variations-Ratio-Index $R$ als 
Neigung ethnischer Gruppen, sich ungleich auf lokale Einheiten wie Schulen oder Regionen aufzuteilen. Ein weiteres verbreitetes statistisches Maß zur Darstellung von Einkommensungleichverteilungen ist der Gini-Koeffizient $G$ nach James und Taeuber (1985). Im Unterschied zum ID reagiert er auch auf Veränderungen in der Berufsstruktur, die sich zwischen stark und weniger stark geschlechtsspezifisch segregierten Berufen ereignen. Der IP-Index von Karmel und Maclachlan (1988) stellt eine Weiterentwicklung des zuvor beschriebenen Dissimilaritätsindizes ID dar unter dem Gesichtspunkt der besseren Interpretierbarkeit. Der Index misst den Anteil der Beschäftigten, die ihren Beruf wechseln müssten, um eine Gleichverteilung der Geschlechter über die Berufe zu erzielen unter Beibehaltung der Berufsstruktur der Beschäftigten. Der Marginal-Matching-Index MM nach Siltanen, Jarman und Blackburn (1995) misst, wie stark Frauenberufe von weiblichen Arbeitskräften und wie stark Männerberufe von männlichen Arbeitskräften ausgeübt werden. Je stärker diese Beziehung, desto höher ist der Grad der Segregation. Hutchens (2001) definiert wünschenswerte Eigenschaften, die ein Index erfüllen sollte und charakterisiert eine Familie von Indizes, die mehrere dieser Eigenschaften erfüllen. Der Square-Root-Index $S$ nach Hutchens (2004) wird mit der Absicht eingeführt, einen Index zu entwickeln, der ein Maximum wünschenswerter Eigenschaften erfüllt. Mit Ausnahme des Informationstheorieindizes $H$ bleibt die Anwendung sämtlicher zuvor beschriebenen Methoden auf binäre Variablen beschränkt. Es kann aber durchaus von Interesse sein, wie gleich bzw. ungleich sich mehr als zwei Gruppen auf unterschiedliche Berufe oder Regionen verteilen.

Eine weitere Möglichkeit zur Messung von Segregation bésteht in der Anwendung des Mutual-Information-Indizes $M$. Er bezieht sich auf den Informationstheorieindex $H$ und wurde als erstes von Theil (1971) vorgeschlagen und von Fuchs (1975) sowie Mora und Ruiz-Castillo (2003, 2004) angewandt, um geschlechtsspezifische Segregation am Arbeitsmarkt zu messen. Mora und Ruiz-Castillo (2007) sowie Frankel und Volij (2008) entwickeln einen additiv zerlegbaren Index $M$, mit dem gemessen werden kann, wie gleich oder ungleich sich ethnische Gruppen auf Schulen über unterschiedliche Regionen verteilen.

Kim und Jargowsky (2005) entwickeln eine allgemeine Form des Gini-Koeffizienten, der als Segregationsmaß sowohl für stetige als auch dichotome Variablen angewandt werden kann. Chakravarty, d'Ambrosio und Silber (2009) beabsichtigen, einen Koeffizienten herzuleiten unter der Annahme, dass die Anzahl der zu vergleichenden Gruppen frei wählbar ist und die Anzahl der Personen in diesen Gruppen auch null sein kann. Der entsprechende Index entspricht ebenfalls einer allgemeinen mehrdimensionalen Form des Gini-Koeffizienten.

Reardon (2008) argumentiert, dass die herkömmlichen Verfahren zur Messung von Segregation auf nominalen Kategorien beruhen. Diese Methoden sind jedoch unangebracht, wenn entweder die Gruppen oder aber die organisatorischen Einheiten (Regionen, Berufe) ordinal skaliert sind und entwickelt Methoden, die in diesem Fall anwendbar sind.

Ziel dieses Beitrags ist, die gängigen Methoden zur Messung von Segregation vorzustel- 
len, zu vergleichen sowie Stärken und Schwächen der einzelnen Verfahren zu diskutieren. Die methodischen Ansätze stehen hierbei im Vordergrund, aber auch die Darstellung empirischer Ergebnisse ist Gegenstand der nachfolgenden Ausführungen. Dieser Artikel ist folgendermaßen gegliedert: In den Abschnitten 2-5 werden die gängigen Indizes vorgestellt und in Abschnitt 6 erfolgt ein abschließendes Resüme.

\subsection{Traditionelle Messverfahren}

\subsubsection{Methodologie}

Im Folgenden entspricht $m_{k} \in\left\{M_{k}, F_{k}\right\}$ der Anzahl der Männer $M_{k}$ bzw. der Anzahl der Frauen $F_{k}$ in Beruf $k, m \in\{M, F\}$ der Gesamtzahl beschäftigter Männer $M$ bzw. Frauen $F$ auf dem Arbeitsmarkt, $\pi_{k m}=\frac{m_{k}}{M_{k}+F_{k}}$ dem Anteil von Gruppe $m$ in Beruf $k, \pi_{m}=\frac{m}{M+F}$ dem Anteil von Gruppe $m$ auf dem Arbeitsmarkt insgesamt, $t_{k}=M_{k}+F_{k}$ der Anzahl der Beschäftigten in Beruf $k$ und $T=M+F$ der Anzahl der Beschäftigten insgesamt.

Der Dissimilaritätsindex ID geht auf Duncan und Duncan (1955) zurück und wurde zur Bestimmung der Wohnungssegregation zwischen der schwarzen und weißen Bevölkerung entwickelt. Er entspricht

$$
I D=\frac{1}{2} \sum_{k=1}^{K}\left|\frac{M_{k}}{M}-\frac{F_{k}}{F}\right|
$$

bzw. nach Taeuber und Taeuber (1965)

$$
I D=\sum_{k=1}^{K} \frac{t_{k}\left|\pi_{k m}-\pi_{m}\right|}{2 T \pi_{m}\left(1-\pi_{m}\right)}
$$

Der $I D$ ist symmetrisch hinsichtlich der beiden Gruppen und insofern ist es egal, ob in Gleichung (2.2) Gruppe $M$ oder $F$ bei der Berechnung herangezogen wird. Er kann interpretiert werden als Prozentsatz von Männern und Frauen, die ihren Beruf wechseln müssten damit sich Männer und Frauen gleich auf die Berufe aufteilen.

Der standardisierte Dissimilaritätsindex $I D_{s t}$ nach Gibbs (1965) und Gross (1968) entspricht

$$
I D=\frac{1}{2} \sum_{k=1}^{K}\left|\frac{F_{k} / t_{k}}{\sum_{k=1}^{K} F_{k} / t_{k}}-\frac{M_{k} / t_{k}}{\sum_{k=1}^{K} M_{k} / t_{k}}\right| .
$$

Er beschreibt ebenfalls den Anteil der Frauen oder Männer, die ihren Beruf wechseln müssten, so dass die Verteilung von Männern und Frauen innerhalb der Berufe ihrer Verteilung im Arbeitsmarkt bzw. in der Belegschaft des Betriebes entspräche, ohne allerdings dabei die Größe des Berufes zu berücksichtigen. Da der Einfluss der Berufsstruktur konstant gehalten wird, sind Vergleiche über die Zeit oder zwischen Betrieben möglich, auch 
wenn sich die Beschäftigtenzahlen in den Berufsgruppen unterschiedlich entwickeln.

Der Sex Ratio $S R$ wird von Hakim (1981) angewandt, um Segregation am Britischen Arbeitsmarkt zu analysieren. Er wird definiert als

$$
H=\frac{F_{f} / T_{f}}{F / T}-\frac{F_{m} / T_{m}}{F / T}
$$

wobei die Indizes $f, m$ Frauen- und Männerberufe kennzeichnen. Beispielsweise stellt $F_{m}$ die Anzahl von Frauen in männerdominierten Berufen dar und $T_{m}$ die Anzahl sämtlicher Erwerbstätigen in männerdominierten Berufen. Ein Beruf wird als frauendominert definiert, wenn der Anteil der Frauen im Beruf größer ist als der Anteil der Frauen auf dem gesamten Arbeitsmarkt, also $F_{k} / t_{k}>F / T$. Die Definition männerdominierter Berufe erfolt analog. Hakim (1981) beschreibt dieses Maß als den Unterschied zwischen dem Ausmaß an Überpräsentation von weiblichen Arbeitskräften in typischen Frauenberufen und Unterpräsentation von weiblichen Arbeitskräften in typischen Männerberufen.

Der IP-Index von Karmel und Maclachlan (1988) stellt eine Weiterentwicklung des zuvor beschriebenen Dissimilaritätsindezes $I D$ dar unter dem Gesichtspunkt der besseren Interpretierbarkeit. Er wird definiert als

$$
I P=\frac{1}{T} \sum_{k=1}^{K}\left|m_{k}-\pi_{m} t_{k}\right| .
$$

Nach Falk (2002) misst der IP-Index den Anteil der Beschäftigten im Gegensatz zu Frauen oder Männern wie beim $I D$, die ihren Beruf wechseln müssten, um eine Gleichverteilung der Geschlechter über die Berufe zu erzielen unter Beibehaltung der Berufsstruktur der Beschäftigten. Die Indizes $I P$ und $I D$ stehen in folgender Beziehung zueinander:

$$
I P=2 \pi_{m}\left(1-\pi_{m}\right) D
$$

Der Marginal-Matching-Index $M M$ nach Siltanen, Jarman und Blackburn (1995) misst, vergleichbar mit $S R$ wie stark Frauenberufe von weiblichen Arbeitskräften und wie stark Männerberufe von männlichen Arbeitskräften ausgeübt werden. Es wird zwischen Frauen- und Männerberufen unterschieden und folgende Segregationsmatrix erzeugt:

$$
\left(\begin{array}{ll}
F_{f} & M_{f} \\
F_{m} & M_{m}
\end{array}\right)
$$

Es gilt nun, eine Scheidelinie so zu ziehen, dass die Segregationsmatrix symmetrisch ist, d.h., dass $M_{f}=F_{m}$ und somit

$$
\sum_{k=1}^{n^{F}}\left(M_{k}+F_{k}\right)=F=\sum_{k=1}^{n} F_{k}
$$


mit $n^{F}$ als Anzahl frauendominierter Berufe. Für den üblichen Fall, dass die Gleichung nicht exakt aufgeht, schlagen Blackburn, Jarman und Siltanen (1993) eine Interpolation vor. Die Scheidelinie variiert je nach der sich verändernden Geschlechterzusammensetzung der Beschäftigung im Zeitverlauf. Der Marginal-Matching-Index $M M$ wird definiert als

$$
M M=\frac{1}{F M}\left(F_{f} M_{m}-F_{m} M_{f}\right)=\frac{F_{f}}{F}-\frac{M_{m}}{M} .
$$

Der Gini-Index G nach James und Taeuber (1985) entspricht

$$
G=\sum_{k=1}^{K} \sum_{s=1}^{K} \frac{t_{k} t_{s}\left|\pi_{k m}-\pi_{s m}\right|}{2 T^{2} \pi_{m}\left(1-\pi_{m}\right)}
$$

und kann interpretiert werden als Summe der gewichteten durchschnittlichen absoluten Differenzen der Anteile einer bestimmten Personengruppe $m$ zwischen allen möglichen Paaren von Berufsgruppen geteilt durch den größtmöglichen Wert dieser Summe, der sich bei vollständiger Segregation ergeben würde. Im Unterschied zum ID reagiert er auch auf Veränderungen in der Berufsstruktur, die sich zwischen stark und weniger stark geschlechtsspezisch segregierten Berufen ereignen.

Der Variations-Ratio-Index $R$ nach James und Taeuber (1985) wird definiert als

$$
R=\sum_{k=1}^{K} \frac{t_{k}\left(\pi_{k m}-\pi_{m}\right)^{2}}{T \pi_{m}\left(1-\pi_{m}\right)}
$$

und kann interpretiert werden als Varianz der Anteile von Arbeitnehmern einer bestimmten Personengruppe $m$ in den Berufen geteilt durch die Varianz der dichotomen Variablen Geschlecht bei sämtlichen Arbeitskräften. Er wird als Neigung von Gruppen definiert, sich ungleich auf Berufe aufzuteilen.

Der Square-Root-Index $S$ nach Hutchens (2004) wird mit der Absicht eingeführt, einen Index zu entwickeln, der ein Maximum wünschenswerter Eigenschaften erfüllt. Er entspricht

$$
S=\sum_{k=1}^{K}\left[\sqrt{\frac{F_{k}}{F} \cdot \frac{M_{k}^{\text {keine Segregation }}}{M}}-\sqrt{\frac{F_{k}}{F} \cdot \frac{M_{k}}{M}}\right]
$$

und kann interpretiert werden als Summe der Unterschiede zwischen den beobachteten Anteilen von Männern und Frauen über die Berufsgruppen hinweg und den Anteilen, die sich bei gleichmäßiger Aufteilung der beiden Gruppen über die Berufe ergeben würden. Für jede Berufsgruppe wird dieser Unterschied definiert als Differenz des geometrischen 
Mittels der Anteile von Männern und Frauen auf die Berufe in Abwesenheit von Segregation und dem geometrischen Mittel der tatsächlich beobachteten Anteile der beiden Geschlechter. Da im Fall nicht vorhandener Segregation $\frac{F_{k}}{F}=\frac{M_{k}}{M}$ gilt stets

$$
S=\sum_{k=1}^{K}\left[\frac{F_{k}}{F}-\sqrt{\frac{F_{k}}{F} \cdot \frac{M_{k}}{M}}\right] .
$$

Mit Ausnahme des $S R$ nehmen sämtliche vorgestellten Indizes den Wert 0 bei nicht vorhandener Segregation und den Wert 1 bei vollständiger Segregation an. Die vorgestellten traditionellen Messverfahren sind nicht für den Mehrgruppenfall geeignet. Sie können auf beliebige Personengruppen und andere organisatorische Einheiten wie z.B Schulen oder Regionen übertragen werden.

\subsubsection{Empirische Resultate}

In der folgenden Studie werden die zuvor beschriebenen Indizes angewandt, um berufliche Segregation zu messen. Für die Segregationsmessung wird das vom Deutschen Institut für Wirtschaftsforschung (DIW) erstellte Sozio-ökonomische Panel (SOEP) verwendet. Es handelt sich hierbei um eine repräsentative Befragung privater Haushalte in Deutschland, die im jährlichen Rhythmus bei denselben Personen und Haushalten seit 1984 durchgeführt wird. Bereits im Juni 1990, also noch vor der Währungs-, Wirtschafts- und Sozialunion, wurde die Studie auf das Gebiet der ehemaligen DDR ausgeweitet.

Bei der Auswahl einer geeigneten Stichprobe werden die selben Kriterien wie bei den Lohndiskriminierungsmessungen herangezogen, d.h., dass sozialversicherungspflichtige Arbeiter, Angestellte und Beamte in Vollzeit- oder Teilzeittätigkeit von 18-65 Jahren aus den zugehörigen SOEP-Wellen herausgefiltert werden. Nicht berücksichtigt sind Selbständige, Praktikanten und Auszubildende, unbezahlt mithelfende Familienangehörige und Personen, die ausschließlich in so genannten Mini-Jobs oder 1-Euro-Jobs tätig sind. Die vorgestellten Studien beziehen sich auf die Jahre 1999 und 2006 für Gesamtdeutschland. Arbeitnehmer mit fehlenden oder unplausiblen Antworten werden wie in vergleichbaren, auf dem SOEP basierenden Analysen (vgl. Melly (2006)) nicht berücksichtigt. Hierbei wird unterstellt, dass keine systematische Antwortverweigerung oder -verfälschung vorliegt. Fehlende Werte sind über sämtliche Beobachtungen zufällig verteilt und somit ist kein Selektionsbias zu erwarten.

Die in dieser Studie gewählten Berufsgruppen unterscheiden nach beruflichem Status, wobei das zugrunde liegende Skalenniveau stark mit der Treimann-Prestige-Skala ${ }^{1}$ korreliert. Es werden folgende Berufsgruppen gewählt:

\footnotetext{
${ }^{1}$ Der Prestigerang von Berufen wird in bildungssoziologischen Untersuchungen als relativ aussagekräftige Kategorie benutzt. Donald J. Treiman entwickelte den Standard Index of Occupational Prestige Scala (SIOPS) (auch Treiman-Index genannt) auf Grundlage des Klassifikationssystems ISCO von 1968.
} 
- Gruppe 1: Tätigkeit mit geringem Status und wenig Eigenständigkeit.

- Gruppe 2: Beschäftigung mit einem geringen Maß an Spezialisierung.

- Gruppe 3: Tätigkeit, die eine gewisse Schulbildung erfordert und ein begrenztes Maß an Verantwortung einschließt.

- Gruppe 4: Leitende aber nicht prestigeträchtige Tätigkeit, die Fachhochschul oder Universitätsabschluss erfordert.

- Gruppe 5: Prestigeträchtige Tätigkeit mit umfassenden Führungsaufgaben.

Die Stichprobe umfasst für das Jahr 1999 bei Männern 1979 Beobachtungen, die der Frauen 1136 Beobachtungen und für das Jahr 2006 bei Männern 3270 Beobachtungen und bei Frauen 3166 Beobachtungen. Tabelle (2.1) zeigt wie sich Frauen und Männer auf die Berufsgruppen aufteilen,

\begin{tabular}{ccccr}
\hline \hline & & & & \\
Jahr & Gruppe & Männer & Frauen & $\sum$ \\
\hline \multirow{2}{*}{2006} & 1 & 522 & 393 & 915 \\
& 2 & 1257 & 835 & 2092 \\
& 3 & 845 & 1637 & 2482 \\
& 4 & 314 & 181 & 495 \\
& 5 & 332 & 120 & 452 \\
& $\sum$ & 3270 & 3166 & 6436 \\
\hline \hline & & & & \\
1999 & 1 & 335 & 149 & 484 \\
& 2 & 645 & 296 & 941 \\
& 3 & 417 & 526 & 943 \\
& 4 & 511 & 148 & 659 \\
& 5 & 71 & 17 & 88 \\
& $\sum$ & 1979 & 1136 & 3115 \\
\hline \hline
\end{tabular}

Tabelle 2.1: Aufteilung von Frauen und Männern auf die Berufsgruppen

Tabelle (2.2) die Ergebnisse der Segregationsmessungen.

\begin{tabular}{cccccccc}
\hline \hline Jahr & $I D$ & $I D_{s} t$ & $S R$ & $I P$ & $G$ & $R$ & $S$ \\
\hline & & & & & & & \\
2006 & 0.259 & 0.198 & 0.555 & 0.129 & 0.291 & 0.076 & 0.039 \\
1999 & 0.252 & 0.219 & 0.759 & 0.117 & 0.298 & 0.076 & 0.041 \\
\hline \hline
\end{tabular}

Tabelle 2.2: Ergebnisse der Segregationsmessungen 
Wird der Gini-Koeffizient $G$ als Maßzahl herangezogen, so fällt die Segregation mit 0.291 (2006) und 0.298 (1999) am Größten, beim Square-Root-Index $S$ mit 0.039 (2006) und 0.041 (1999) am Geringsten aus. Die Werte des Sex Ratios können nicht mit den anderen Indizes verglichen werden, da es sich hierbei um ein nicht normiertes Maß handelt. Die Indexwerte bleiben im Zeitverlauf relativ konstant. Während Segregationsmessungen auf Basis des ID oder IP zu dem Ergebnis kommen, dass die Segregation im Zeitverlauf geringfügig zugenommen hat, bleiben die anderen Maßzahlen konstant oder gehen geringfügig zurück.

\subsection{Auf Entropie basierende Verfahren zur Messung von Segregation}

\subsubsection{Der Informationstheorieindex als Maß für Segregation}

\section{Methodologie}

Beim Informationstheorieindex $H$ nach Theil (1972) wird die Entropie $E$ als Maß für die Ungleichverteilung von Männern und Frauen auf die Berufe herangezogen. Es gilt für die Entropie insgesamt

$$
E=\sum_{k=1}^{K} \pi_{k} \log _{2} \frac{1}{\pi_{k}}
$$

bzw. für die Entropie $E_{m}$ in den einzelnen Personengruppen

$$
E_{m}=\sum_{k=1}^{K} \pi_{m k} \log _{2} \frac{1}{\pi_{m k}}
$$

mit $\pi_{k}=\frac{t_{k}}{T}$ dem Anteil von in Berufsgruppe $k$ beschäftigter Arbeitnehmer auf dem Arbeitsmarkt insgesamt und $\pi_{m k}=m_{k} / m$ dem Anteil von in Beruf $k$ beschäftigter Erwerbstätiger in Gruppe $m 2^{2}$ Die Entropie nimmt den Wert 0 bei nur einer Berufsgruppe an und erreicht ihr Maximum, wenn die Anteile der Individuen auf die Berufe bei den ethnischen Gruppen identisch sind. Der Informationstheorieindex $H$ wird definiert als

$$
H=\sum_{i=1}^{M} \frac{m_{i}\left(E-E_{m}\right)}{T E},
$$

mit $M \geq 2$ Personengruppen. Er kann auf beliebige Personengruppen und andere organisatorische Einheiten wie z.B Schulen oder Regionen übertragen werden.

\footnotetext{
${ }^{2}$ Man definiert $0 \cdot \log _{2}(0)=\lim _{\pi \rightarrow 0} \pi \log _{2} \pi=0$
} 


\subsubsection{Der Mutual-Information-Index als Maß für Segregation}

\section{Methodologie}

Frankel und Volij (2008) entwickeln einen additiv zerlegbaren Segregationsindex ${ }^{3}$, mit dem gemessen werden kann, wie gleich oder ungleich sich ethnische Gruppen auf Schulen über unterschiedliche Regionen verteilen. Im Folgenden soll gelten: $G$ sei eine diskrete Zufallsvariable mit $M$ möglichen Realisationen und $p_{m}$ die zugehörige Wahrscheinlichkeit. Sei beispielsweise $G$ die ethnische Gruppe eines zufällig ausgewählten Schülers, dann entspricht $p_{m}$ dem Anteil der Schüler der $m$-ten Gruppe in einer bestimmten Region. Als Maß für die Ungleichverteilung der ethnischen Gruppen wird die bereits in Gleichung (2.15) beschriebene Entropie $E=\sum_{m=1}^{M} \pi_{m} \log _{2} \frac{1}{\pi_{m}}$ herangezogen. Im Folgenden sei die ethnische Gruppe, der ein Schüler angehört, nicht bekannt, dafür aber die Schule $n$, die er besucht. Wenn Segregation hinsichtlich der ethnischen Verteilung zwischen den Schulen $N$ in der Region vorliegt, können daraus zusätzliche Informationen über die Gruppenzugehörigkeit gezogen werden. Als Maß für den erwarteten Informationsgewinn zwischen $G$ und $N$ wird die gewichtete Summe der Entropien innerhalb der Schulen definiert, wobei die Gewichtung durch den Anteil der Schüler in den Schulen erfolgt. Der Mutual-Information-Index $M$ entspricht

$$
M=\left(\begin{array}{c}
\text { Entropie der } \\
\text { ethnischen Verteilung } \\
\text { in der Region }
\end{array}\right)-\sum_{\substack{\text { Schulen } n \\
\text { in der Region }}}\left(\begin{array}{c}
\text { Anteil der } \\
\text { Schüler in } \\
\text { Schule } n
\end{array}\right)\left(\begin{array}{c}
\text { Entropie der } \\
\text { ethnischen Verteilung } \\
\text { in Schule } n
\end{array}\right)
$$

dh. der Minderung der Ungewissheit über die ethnische Herkunft eines Schülers aus der Kenntnis heraus, welche Schule dieser besucht. Da der Mutual-Information-Index $M$ symmetrisch bezüglich der beiden Variablen $G$ und $N$ ist, gilt

$$
M=\left(\begin{array}{c}
\text { Entropie der } \\
\text { Schulverteilung } \\
\text { in der Region }
\end{array}\right)-\sum_{\text {ethnische Gruppen } g}\left(\begin{array}{c}
\text { Anteil der } \\
\text { Schüler in } \\
\text { Gruppe } g
\end{array}\right)\left(\begin{array}{c}
\text { Entropie der } \\
\text { Schulverteilung } \\
\text { in Gruppe } g
\end{array}\right) .
$$

Der Informationstheorieindex $H$ nach Gleichung (2.16) und der Mutual-InformationIndex $M$ nach Gleichung 2.17) stehen in folgender Beziehung zueinander:

$$
H=M /\left(\begin{array}{c}
\text { Entropie } \\
\text { innerhalb } \\
\text { der Region }
\end{array}\right)
$$

\footnotetext{
${ }^{3}$ Mit additiver Zerlegbarkeit sind starke Schul- und Gruppenzerlegbarkeit (vgl. Gleichungen 2.21) und 2.22) gemeint
} 
Während $H$ nur Werte zwischen 0 und 1 anehmen kann, ist der Wertebereich für $M$ nach oben nicht beschränkt. Im Folgenden soll gelten:

Definition 1: Eine Schulregion $X$ setzt sich zusammen aus:

- Einer nichtleeren und endlichen Menge von ethnischen Gruppen $\boldsymbol{G}(X)$.

- Einer nichtleeren und endlichen Menge von Schulen $\boldsymbol{N}(X)$.

- Für jede ethnische Gruppe $g \in \boldsymbol{G}(X)$ und jede Schule $n \in \boldsymbol{N}(X)$ eine nichtnegative Anzahl $T_{g}^{n}$ von Mitgliedern in Gruppe $g$, die Schule $n$ besuchen.

Eine Schulregion kann auch im Listenformat $\left\langle\left(T_{g}^{n}\right)_{g \in \boldsymbol{G}}\right\rangle_{n \in \boldsymbol{N}}$ spezifiziert werden. Beispielsweise bezeichnet $\langle(10,20),(30,10)\rangle$ eine Region mit zwei ethnischen Gruppen und zwei Schulen. Die erste Schule $(10,20)$ umfasst 10 Inländer und 20 Ausländer, die zweite Schule $(30,10) 30$ Inländer und 10 Ausländer.

Für zwei beliebige Schulregionen $X$ und $Y$ bezeichnet $X \uplus Y$ den Zusammenschluss der Schulen in $X$ und der Schulen in $Y$ in einer Region. Ist $\alpha$ ein nichtnegativer Skalar, so meint $\alpha X$, dass die Anzahl der Schüler in jeder Gruppe und Schule in Region $X$ mit $\alpha$ multipliziert wird. $c(X)$ entspricht dem Zusammenschluss aller Schulen in $X$ zu einer Schule. Ist beispielsweise $X=\langle(10,20),(30,10)\rangle$ und $Y=\langle(40,50)\rangle$, so ergibt $X \uplus Y=\langle(10,20),(30,10),(40,50)\rangle, 2 X=\langle(20,40),(60,20)\rangle$ und $c(X)=\langle(40,30)\rangle$.

Es wird folgende Ausdrucksweise gewählt:

- $T_{g}=\sum_{n \in \boldsymbol{N}} T_{g}^{n}$ : Anzahl der Schüler in Gruppe $g$ in einer Region

- $T^{n}=\sum_{g \in \boldsymbol{G}} T_{g}^{n}:$ Anzahl der Schüler, die Schule $n$ besuchen

- $T=\sum_{g \in \boldsymbol{G}} T_{g}$ : Anzahl der Schüler insgesamt in einer Region

Im Folgenden sei $C$ die Menge aller Schulregionen. Eine Segregationsrelation $\succeq$ sei eine zweistellige, transitive und totale Relation auf $C$. Die Beziehung $X \succeq Y$ meint, dass Region $X$ mindestens genauso segregiert ist, wie Region $Y$. Die Relationen $\succ$ und $~$ werden von $\succeq$ auf die übliche Weise abgeleitet 4 . Der Segregationsindex ist eine Funktion $S: C \rightarrow R$, d.h., dass jeder Region aus $C$ eine reelle Zahl zugeordnet wird. Der Index $S$ repräsentiert die Segregationsrelation $\succeq$ für alle Paare von Regionen $X, Y \in C$ :

$$
X \succeq Y \Longleftrightarrow S(X) \geq S(Y)
$$

Wird die Transformation $\sigma: C \rightarrow C$ auf eine Schulregion $X$ angewandt, so bleibt die Segregation in der Region $X$ unverändert, d.h., dass $\sigma(X) \sim X$. Gilt diese Beziehung für

\footnotetext{
${ }^{4} x \sim y$, wenn $X \succeq Y$ und $Y \succeq X, X \succ Y$, wenn $X \succeq Y$ aber nicht $Y \succeq X$
} 
sämtliche Regionen, so ist die Segregation invariant gegenüber der Transformation $\sigma$.

Nach Frankel und Volij (2008) erfüllt der Mutual-Information-Index $M$ folgende wünschenswerte Eigenschaften:

- Symmetrie: Die Segregation in einer Region ist invariant gegenüber Umbenennungen oder Neusortierungen von Gruppen und Schulen.

- Schulspaltung: Es sei $\boldsymbol{N}$ die Menge aller Schulen in der Region $X \in C$ und $X^{\prime}$ das Ergebnis, wenn $n \in \boldsymbol{N}$ Schulen in zwei Schulen $n_{1}$ und $n_{2}$ aufgespalten werden. Dann gilt $X^{\prime} \succeq X$. Haben die beiden Schulen dieselbe ethnische Verteilung, dann gilt $X^{\prime} \sim X$.

- Typ-1-Unabhängigkeit: Es seien $X, Y \in C$ zwei Regionen mit identischer Anzahl an Schülern und denselben ethnischen Verteilungen, wobei $Y$ dabei als Neuverteilung der Schüler auf die Schulen angesehen werden kann ${ }^{5}$ Durch das Hinzufügen eines Clusters $Z$ bestehend aus nur einer Schule soll gelten $X \succeq Y$ genau dann, wenn $X \uplus$ $Z \succeq Y \uplus Z$, d.h., dass durch das Hinzufügen eines Clusters $Z$ bestehend aus nur einer Schule nicht beeinflusst wird, welche Region stärker segregiert. Der $I D$ nach Duncan und Duncan (1955) verletzt dieses Prinzip. Beispielsweise sei $X=\langle(50,100),(50,0)\rangle$, $Y=\langle(100,40),(0,60)\rangle$ und $Z=\langle(100,0)\rangle$. Es gilt: $I D(X)=0.5<I D(Y)=0.6$, aber $I D(X \uplus Z)=0.75>I D(X \uplus Y)=0.6$. Wird als Segregationsmaß der MutualInformation-Index herangezogen, so ist für das gewählte Beispiel $M(X)=0.311<$ $M(Y)=0.396$ und $M(X \uplus Z)=0.459<M(X \uplus Y)=0.516$, womit Typ-1Unabhängigkeit erfüllt ist.

- Typ-2-Unabhängigkeit: Es seien $X, Y, Z \in C$ drei Regionen mit $T(X)=T(Y)$, d.h., dass die Anzahl der Schüler in $X$ und $Y$ identisch ist. Weiterhin sei das Cluster $c(Z)$ der Zusammenschluss aller Schulen in $Z$ zu einer Schule. Es soll gelten $X \uplus Z \succeq Y \uplus Z$ genau dann, wenn $X \uplus c(Z) \succeq Y \uplus c(Z)$. Die meisten Indizes verletzen dieses Prinzip, da sie nicht zwischen Segreation zwischen Clustern und innerhalb eines Clusters unterscheiden. Beispielsweise sei $X=\langle(50,0),(0,100)\rangle, Y=\langle(100,0),(0,50)\rangle$ und $Z=\langle(50,0),(0,100)\rangle$. Die ethnische Verteilung in den Schulregionen $X$ und $Z$ ist identisch mit jeweils 50 In- und 100 Ausländern und weicht von $Y$ ab. Demzufolge ist die Segregation zwischen den Clustern $X$ und $Z$ geringer als zwischen $Y$ und $Z$. Allerdings berücksichtigen die meisten Indizes nur Segregation innerhalb eines Clusters. Wird als Segregationsmaß der ID nach Duncan und Duncan (1955) herangezogen, werden die Cluster $X \uplus Z$ und $Y \uplus Z$ als maximal und identisch segregiert angesehen mit $I D(X \uplus Z)=I D(Y \uplus Z)=1$. Für das gewählte Beispiel ergibt sich $I D(X \uplus c(Z))=0.5<I D(Y \uplus c(Z))=2 / 3$, womit Typ-2-Unabhängigkeit verletzt ist. Bei Verwendung des Mutual-Information-Indizes gilt: $M(X \uplus Z)=0.981<$ $M(Y \uplus Z)=1$ und $M(X \uplus c(Z))=0.459<M(Y \uplus c(Z))=0.541$, womit Typ-2Unabhängigkeit erfüllt ist.

\footnotetext{
${ }^{5}$ Typ-1-Unabhängigkeit setzt nicht voraus, dass die Anzahl der Schulen in $X$ und $Y$ identisch ist.
} 
- Gruppenspaltung: Es sei $\boldsymbol{G}$ die Menge der ethnischen Gruppen in einer Region

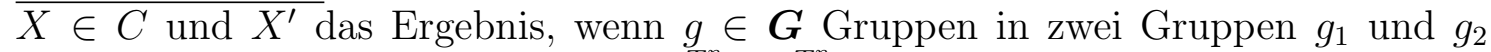
aufgespalten werden in der Art, dass $\frac{T_{g 1}^{n}}{T_{g 1}}=\frac{T_{g 2}^{n}}{T_{g 2}}$ für alle $n \in \boldsymbol{N}$. Dann ist $X \sim X^{\prime}$.

- Größeninvarianz: Wenn die Anzahl der Schüler in jeder ethnischen Gruppe und jeder Schule $n$ mit derselben positiven Konstanten $\alpha$ multipliziert wird, dann gilt für jede Region $X \in C$ und jeden Skalar $\alpha$, dass $X \sim \alpha X$.

- Skaleninvarianz: Sei $X^{\prime}$ das Ergebnis, wenn die Anzahl der Schüler einer bestimmten ethnischen Gruppe $g \in \boldsymbol{G}(X)$ in jeder Schule $n$ mit derselben positiven Konstanten $\alpha$ multipliziert wird. Dann gilt $X^{\prime} \sim \alpha X$.

- Starke Schulzerlegbarkeit: Bei jeder beliebigen Aufteilung $X=X^{1} \uplus \ldots \uplus X^{K}$ der Schulen einer Region in $K$ Cluster gilt

$$
S(X)=S\left(c\left(X^{1}\right) \uplus \ldots \uplus c\left(X^{K}\right)\right)+\sum_{k=1}^{K} P^{k} S\left(X^{k}\right)
$$

mit $P^{k}$ gleich dem Anteil der Schüler in Cluster $k$, wobei $S\left(c\left(X^{1}\right) \uplus \ldots \uplus c\left(X^{K}\right)\right)$ der Segregation zwischen den $K$ Clustern und $S\left(X^{k}\right)$ der Segregation innerhalb des Clusters $k$ entspricht. Dieses Prinzip wird von den meisten Indizes verletzt. ${ }^{6}$

- Starke Gruppenzerlegbarkeit: Bei jedem beliebigen Zusammenschluss von ethnischen Gruppen einer Region in $K$ Supergruppen gilt

$$
S=S_{K}+\sum_{k=1}^{K} P^{k} S_{k}
$$

mit $P_{k}$ gleich dem Anteil der Schüler in Supergruppe $k . S_{K}$ entspricht der Segregation der Region, wenn jede Supergruppe wie eine einzelne Gruppe behandelt wird und $S_{k}$ der Segregation der Region, wenn alle Schüler, die nicht der Supergruppe angehören getrennt behandelt werden. Dieses Prinzip soll an folgendem Beispiel erläutert werden: In einer Region wird zwischen vier ethnischen Gruppen A, B, C und D unterschieden, wobei A, B und C zu einer Supergruppe zusammengefasst werden. Unter zweimaliger Verwendung von Gleichung (2.22) kann die Segregation in drei Terme zerlegt werden:

$$
\begin{aligned}
\left(\begin{array}{c}
\text { Segregation } \\
\text { zwischen } \\
\mathrm{A}, \mathrm{B}, \mathrm{C} \& \mathrm{D}
\end{array}\right)= & \left(\begin{array}{c}
\text { Segregation } \\
\text { zwischen } \\
\{\mathrm{A}, \mathrm{B}, \mathrm{C}\} \& \mathrm{D}
\end{array}\right)+\left(\begin{array}{c}
\text { Anteil der } \\
\text { Schüler in } \\
\mathrm{A}, \mathrm{B}, \text { oder } \mathrm{C}
\end{array}\right)\left(\begin{array}{c}
\text { Segregation } \\
\text { zwischen } \\
\mathrm{A} \&\{\mathrm{~B}, \mathrm{C}\}
\end{array}\right) \\
& +\left(\begin{array}{c}
\text { Anteil der } \\
\text { Schüler in } \\
\{\mathrm{B}, \mathrm{C}\}
\end{array}\right)\left(\begin{array}{c}
\text { Segregation } \\
\text { zwischen } \\
\mathrm{B} \& \mathrm{C}
\end{array}\right)
\end{aligned}
$$

${ }^{6}$ Siehe Empirische Resultate 
Starke Gruppenzerlegbarkeit wird ebenfalls von den meisten Indizes nicht erfüllt.

- Stetigkeit: Für drei beliebige Regionen $X, Y, Z \in C$ stellen $\{c \in[0,1]: c X \uplus(1-$ $\overline{c)} Y \succeq Z\}$ und $\{c \in[0,1]: Z \succeq c X \uplus(1-c) Y\}$ abgeschlossene Mengen dar. Dieses Axiom ist technischer Natur und wird benötigt, um zu beweisen, dass die Segrationsrelation durch einen Index repräsentiert wird.

- Nichttrivialität: Es existieren zwei Regionen $X, Y \in C$ mit zwei nichtleeren ethnischen Gruppen, die nicht identisch segregiert sind, also $X \succ Y$. Dieses Axiom wird benötigt, um die triviale Segregationsrelation auszuschließen.

\subsubsection{Empirische Resultate}

In der folgenden Studie wird die vom Landesbetrieb für Statistik und Kommunikationstechnologie Niedersachsen bereitgestellte und frei verfübgare LSKN-Online Datenbank herangezogen. Die betrachteten Schulegionen sind die Samtgemeinden Nenndorf und Rodenberg im Landkreis Schaumburg in Niedersachsen. Bei den Schulen werden Grundschulen ausgewählt, da sich diese im Gegensatz zu anderen Schulformen in beiden Gemeinden befinden. Bei den ethnischen Gruppen wird zwischen In- und Ausländern unterschieden. Die Spezifikation für das Jahr 2010 lautet im Listenformat:

- $N=\langle(337,26),(240,2)\rangle$ für die Santgemeinde Nenndorf

- $R=\langle(364,12),(278,13)\rangle$ für die Samtgemeinde Rodenberg

- $N \uplus R=\langle(337,26),(240,2),(364,12),(278,13)\rangle$ für den Zusammenschluss beider Gemeinden

Segregation wird zunächst für beide Gemeinden seperat ermittelt und anschließend für den Zusammenschluss beider Gemeinden in einer Region. Tabelle (2.3) zeigt die Ergebnisse der Segregationsmessungen,

\begin{tabular}{lcc}
\hline \hline Region & $H$ & $M$ \\
\hline$N$ & 0.072 & 0.020 \\
$R$ & 0.003 & 0.001 \\
$N \uplus R$ & 0.040 & 0.010 \\
\hline \hline
\end{tabular}

Tabelle 2.3: Ergebnisse der Segregationsmessungen

wobei unter Verwendung des Mutual-Information-Indizes $S(N \uplus R)$ auch gemäß Gleichung (2.21) ermittelt werden kann. Bei nur zwei Schulregionen $N$ und $R$ gilt: 


$$
S(N \uplus R)=S(c(N) \uplus c(R))+P^{R} S(R)+P^{N} S(N)
$$

mit $c(N) \uplus c(R)=\langle(577,28)\rangle \uplus\langle(642,25)\rangle=\langle(577,28),(642,25)\rangle$.

Die Schulsegregation ist bei beiden Indizes in der Gesamtgemeinde Rodenberg (0.003 bzw. 0.001) geringer als in der Gesamtgemeinde Nenndorf (0.072 bzw. 0.020) und liegt für den Zusammenschluss beider Gemeinden (0.040 bzw. 0.010) dazwischen.

\subsection{Der Gini-Koeffizient als Maß für Segregation bei stetigen Variablen}

\subsubsection{Methodologie}

Der Gini-Koeffizient $G$ ist ein weit verbreitetes statistisches Maß zur Darstellung von Einkommensungleichverteilungen. Er wird ebenfalls als Segregationsmaß verwendet, obwohl seine Anwendung in diesem Zusammenhang im Allgemeinen auf binäre Variablen beschränkt bleibt. Kim und Jargowsky (2005) entwickeln eine modifizierte Form dieses Indizes, der auch als Segregationsmaß für stetige Variablen angewandt werden kann.

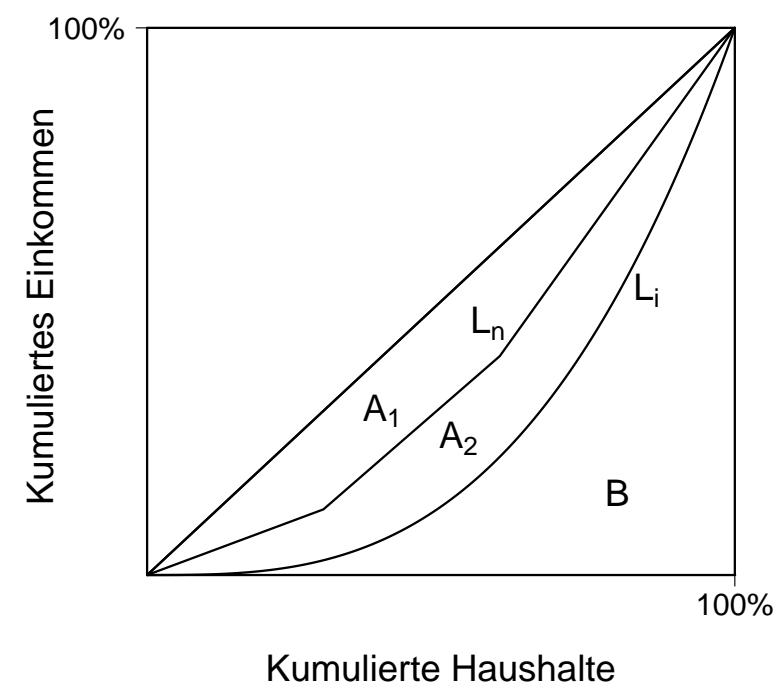

Abbildung 2.1: Lorenzkurven für individuelle Haushalte und Regionen

Wie in Abbildung (2.1) dargestellt, spiegelt die Lorenzkurve den kumulierten Prozentsatz des gesamten Haushaltseinkommens, geordnet nach der Größe des Einkommens, als Funktion des kumulierten Anteils an den gesamtem Haushalten wieder. Der Gini-Koeffizient 
der Einkommensungleichverteilung wird definiert als Fläche zwichen Lorenzkurve und Winkelhalbierender geteilt durch die gesamte Fläche unterhalb der Winkelhalbierenden. In Abbildung (2.1) stellt $L_{i}$ die Lorenzkurve für die individuellen Haushaltseinkommen und $L_{n}$ die Lorenzkurve für die mittleren Einkommen in unterschiedlichen Regionen dar. Der Gini-Koeffizient für die individuellen Haushalte entspricht

$$
G^{i}=\left(A_{1}+A_{2}\right) /\left(A_{1}+A_{2}+B\right)
$$

und für die Regionen

$$
G^{n}=A_{1} /\left(A_{1}+A_{2}+B\right) .
$$

Nach Kim und Jargowsky (2005) entspricht Segregation dem Ausmaß der Ungleichheit zwischen Gruppen bei gegebener Verteilung der individuellen Einheiten. Sie geben zu bedenken, dass der Index $G^{n}$ als Segregationsmaß ungeeignet ist. Zum Einen misst er lediglich die Ungleichheit zwischen Gruppen und berücksichtigt dabei nicht, wie sich die Verteilung der Individuen ändert, wenn individuelle Einheiten zu Gruppen zusammengefasst werden. Zum Anderen hängt die obere Grenze des Gini-Koeffizienten $G^{n}$ ebenfalls von der Verteilung der individuellen Einheiten ab. Bei maximaler Segregation - Haushalte verteilen sich auf Regionen in der Art, dass in einer Region nur Haushalte mit demselben Einkommen vorkommen - konvergiert die Lorenzkuve für Haushalte $L_{n}$ gegen die Lorenzkurve für Individuen $L_{i}$ und die obere Grenze von $G^{n}$ wird durch $G^{i}$ festgelegt. Wird in allen Regionen dasselbe durchschnittliche Einkommen erzielt, nimmt $G^{n}$ den Wert null an. In Anlehnung an Jahn, Schmid und Schrag (1947) schlagen Kim und Jargowsky (2005) vor, bei der Messung von Einkommenssegregation zwischen Regionen die Einkommensungleichverteilung zwischen individuellen Haushalten mit zu berücksichtigen und den Koeffizienten $G^{n}$ neu zu skalieren. Sie definieren

$$
G^{S}=\frac{G^{n}}{G^{i}}=\frac{A_{1} /\left(A_{1}+A_{2}+B\right)}{\left(A_{1}+A_{2}\right) /\left(A_{1}+A_{2}+B\right)}=\frac{A_{1}}{A_{1}+A_{2}},
$$

wobei $G^{S}$ ebenfalls Werte zischen null bei nicht vorhandener Segregation und eins bei maximaler Segregation annehmen kann.

Aus Abbildungen (2.1) und 2.2 geht hervor, dass

$$
\begin{aligned}
G^{i} & =\left(A_{1}+A_{2}\right) /\left(A_{1}+A_{2}+B\right) \\
& =(0.5-B) / 0.5 \\
& =1-2 B \\
2 B & =\sum_{i=1}^{N}\left(Y_{i-1}+Y_{i}\right)\left(H_{i}-H_{i-1}\right) .
\end{aligned}
$$

$Y_{i}$ stellt den kumulierten Prozentsatz der Haushaltseinkommen und $H_{i}$ den kumulierten Anteil an den gesamtem Haushalten dar, aufsteigend geordnet von 1 bis $N$ nach der Höhe 


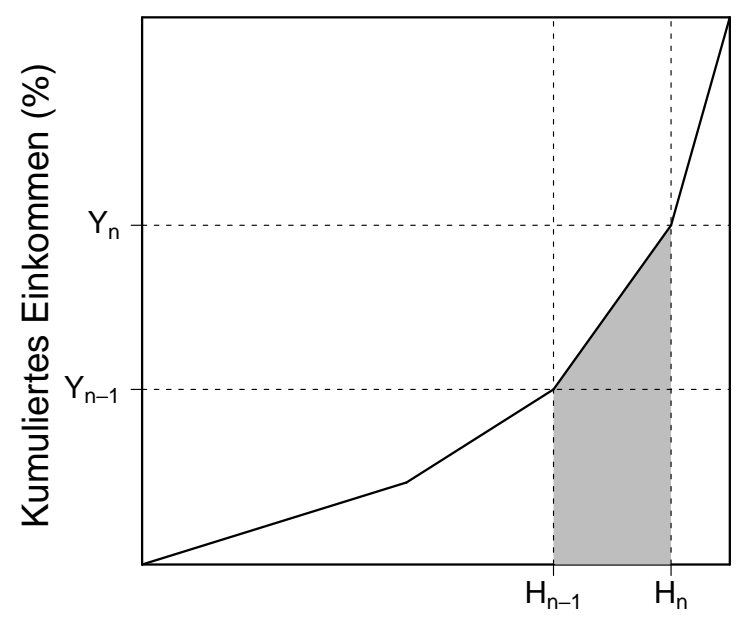

Kumulierte Haushalte (\%)

Abbildung 2.2: Kumuliertes Haushaltseinkommen

des Einkommens, wobei $N$ der Anzahl der Haushalte insgesamt entspricht. $G^{n}$ wird auf analoge Weise ermittelt, wobei die Haushalte nun aufsteigend nach der Höhe des mittleren Einkommens in den Regionen geordnet sind und $Y_{n i}$ den kumulierten Prozentsatz der durchschnittlichen Einkommen darstellt. Es gilt $H_{i}-H_{i-1}=1 / N$ und somit ist

$$
\begin{aligned}
G^{S}=\frac{G^{n}}{G^{i}}= & \frac{1-\sum_{i=1}^{N}\left(Y_{n i-1}+Y_{n i}\right)\left(H_{i}-H_{i-1}\right)}{1-\sum_{i=1}^{N}\left(Y_{i-1}+Y_{i}\right)\left(H_{i}-H_{i-1}\right)} \\
= & \frac{N-\sum_{i=1}^{N}\left(Y_{n i-1}+Y_{n i}\right)}{N-\sum_{i=1}^{N}\left(Y_{i-1}+Y_{i}\right)} .
\end{aligned}
$$

Nach Anand (1983) kann Gleichung (2.29) wie folgt umgeformt werden:

$$
G^{S}=\frac{G^{n}}{G^{i}}=\frac{\frac{1}{2 N^{2} m} \sum_{i=1}^{N} \sum_{j=1}^{N}\left|y_{n i}-y_{n j}\right|}{\frac{1}{2 N^{2} m} \sum_{i=1}^{N} \sum_{j=1}^{N}\left|y_{i}-y_{j}\right|}
$$

wobei $y_{i}$ dem Einkommen des $i$ ten Haushalts, $y_{n i}$ dem durchschnittliche Haushaltseinkommen der Region, in dem sich der Haushalt befindet und $m$ dem mittleren Einkommen 
sämtlicher Haushalte entspricht. Die Gleichungen 2.29) und 2.30 können nicht nur für stetige sondern auch für binäre Variablen angewandt werden, was an folgendem Beispiel verdeutlicht werden soll: Eine Grundgesamtheit $T$ setzt sich aus $F$ Frauen und $M$ Männern zusammen $(T=F+M)$, wobei jeder Frau der Wert eins und jedem Mann der Wert null zugeordnet wird. Kim und Jargowsky (2005) definieren $P=M / T$ und fassen die Abzisse der Lorenzkurve als Prozentsatz der Gesamtbevölkerung und die Ordinate als Prozentsatz der Männer auf. In Gleichung 2.30 wird $m$ durch $P$ ersetzt, $y_{i}$ ist gleich eins bei Frauen und null bei Männern, $y_{n i}$ entspricht $P_{n i}$, dem Anteil der Frauen in der Region, in der sich der i-te Haushalt befindet. Der Nenner $G^{i}$ in Gleichung 2.30 wird $1-P$ und somit gilt

$$
G^{S}=\frac{\sum_{i=1}^{N} \sum_{j=1}^{N}\left|P_{n i}-P_{n j}\right|}{2 N^{2} P(1-P)} .
$$

Gleichung (2.31) wird in der Literatur häufig bei binären Variablen verwendet und entspricht Gleichung (2.10), Gleichungen (2.29) und (2.30) stellen eine Verallgemeinerung dar.

\subsubsection{Empirische Resultate}

Bei der folgenden Untersuchung wird erneut auf das Sozio-ökonomische Panel (SOEP) zurückgegriffen. Es wird zwischen Haushalten in unterschiedlichen Regionen in Gesamtdeutschland unterschieden, wobei sich die Segregationsmessungen auf die Jahre 1999 und 2006 beziehen. Innerhalb dieses Zeitraums sind unterschiedliche politische Maßnahmen durchgeführt worden, die das Ausmaß der Segregation beinflussen können. Arbeitsmarktpolitisch wurden einzelne Gesetze zur Reform des Arbeitsmarktes mit den Kurbezeichnungen Hartz I, Hartz II, Hartz III und Hartz IV umgesetzt. Steuerpolitisch wurden u.a. Eingangs- und Spitzensteuersatz kontinuierlich herabgesetzt. Weiterhin kann das Maß der Einkommensungleichverteilung von Veränderungen der Haushaltszusammensetzungen und einer im betrachteten Zeitraum gesunkenen Arbeitslosenquote abhängen.

In dieser Studie wird zwischen folgenden Regionen unterschieden:

- Nord: Schleswig-Holstein, Hamburg, Niedersachsen und Bremen.

- Süd: Hessen, Baden-Württemberg und Bayern.

- West: Nordrhein-Westfalen, Rheinland-Pfalz und Saarland .

- Ost: Berlin, Brandenburg, Mecklenburg-Vorpommern, Sachsen, Sachsen-Anhalt und Thüringen.

Das Haushaltseinkommen entspricht dem jährlichen Nettohaushaltseinkommen nach Steuern und staatlichen Transferzahlungen sämtlicher Mitglieder eines Haushaltes, wobei das 
Einkommen für das Jahr 1999 entsprechend dem vom Statistischen Bundesamt erhobenen Verbraucherpreisindex 7 auf das Jahr 2006 umgerechnet wurde.

\begin{tabular}{cccccc}
\hline \hline \multirow{2}{*}{ Jahr } & & Nord & Süd & West & Ost \\
\hline \multirow{2}{*}{2006} & Anzahl & 1697 & 3876 & 3067 & 3063 \\
& Einkommen & 35052 & 36595 & 35561 & 27087 \\
\hline \multirow{2}{*}{1999} & Anzahl & 1852 & 4368 & 3637 & 3401 \\
& Einkommen & 31224 & 32200 & 30811 & 26386 \\
\hline \hline
\end{tabular}

Tabelle 2.4: Aufteilung von Haushalten auf die Regionen

Tabelle (2.4) zeigt, wie sich die Haushalte in der Stichprobe auf die unterschiedlichen Regionen in Gesamtdeutschland aufteilen mit den zugehörigen mittleren Haushaltseinkommen. In sämtlichen Regionen ist das Realeinkommen im Zeitverlauf angestiegen, wobei der Zuwachs in den neuen Bundesländern am Kleinsten ausfällt. Zu beiden Zeitpunkten ist das Einkommen im Süden am Höchsten und im Osten am Geringsten.

\begin{tabular}{cccc}
\hline \hline Jahr & $G^{i}$ & $G^{n}$ & $G^{S}$ \\
\hline & & & \\
2006 & 0.373 & 0.056 & 0.151 \\
1999 & 0.324 & 0.039 & 0.119 \\
\hline \hline
\end{tabular}

Tabelle 2.5: Ergebnisse der Segregationsmessungen

Tabelle 2.5 zeigt die Ergebnisse der Segregationsmessungen. Der Gini-Koeffizient $G^{i}$ für die Einkommen der individuellen Haushalte steigt im betrachteten Zeitraum von 0.324 auf 0.373, der Gini-Koeffizient $G^{n}$ für die mittleren Einkommen der Haushalte in den Regionen von 0.039 auf 0.056 und der Segregationsindex $G_{S}$ von 0.119 auf 0.151.

\subsection{Methoden zur Messung ordinaler Regression}

\subsubsection{Methodologie}

Traditionelle Methoden zur Messung von Segregation beschreiben, wie sich nominal skalierte Gruppen (z.B. Männer und Frauen) auf ebenfalls nominal skalierte organisatorische Einheiten (z.B. Regionen, Schulen) verteilen. Reardon (2008) entwickelt einen allgemeinen

\footnotetext{
${ }^{7}$ DESTATIS 2009, Verbraucherprisindex für Deutschland, Lange Reihe ab 1948-Juni 2009
} 
Ansatz, mit dem Segregation gemessen werden kann, wenn entweder die Gruppen oder die organisatorischen Einheiten ordinal skaliert sind.

Zur Motivation soll folgendes Beispiel dienen: Auf einem Arbeitsmarkt befinden sich 100 männliche und 100 weibliche Arbeitskräfte, die sich unterschiedlich auf vier Berufgsgruppen verteilen. Die Berufsgruppen unterscheiden nach beruflichem Status, ansteigend von Kategorie eins bis vier. In Szenario A befinden sich Männer überproportional in Berufen mit hohem Status, Frauen dagegen überproportional in Berufen mit niedrigem Status. In Szenario B tauschen bei beiden Geschlechtern die Verteilungen auf die Berufsgruppen 1 und 2 genauso wie die Verteilungen auf die Gruppen 3 und 4 (siehe Tabelle 2.6). Ein traditionelles Segregationsmaß wie beispielsweise der Dissimilaritätsindex nach Duncan und Duncan (1955) würde die Berufsgruppen kategorial behandeln und beide Szenarien gleich einschätzen. In jedem Szenario gibt es eine Berufsgruppe mit einem 40/10 Männer/Frauen Verhältnis, eine mit einem 30/20 Männer/Frauen Verhältnis, eine mit einem 20/30 Männer/Frauen Verhältnis und mit einem 10/40 Männer/Frauen Verhältnis. Ziel des Ansatzes nach Reardon (2008) ist deshalb, ein geeignetes Maß zu finden, wie gleich oder ungleich sich ungeordnete Gruppen von Arbeitnehmern auf geordnete Berufsgruppen aufteilen.

\begin{tabular}{lccccc}
\hline \hline \multicolumn{5}{c}{ beruflicher Status } \\
& 1 & 2 & 3 & 4 & insgesamt \\
\hline \multicolumn{6}{c}{ Szenario A } \\
Männer & 10 & 20 & 30 & 40 & 100 \\
Frauen & 40 & 30 & 20 & 10 & 100 \\
\hline insgesamt & 50 & 50 & 50 & 50 & 200 \\
\hline \multicolumn{6}{c}{ Szenario B } \\
Männer & 20 & 10 & 40 & 30 & 100 \\
Frauen & 30 & 40 & 10 & 20 & 100 \\
\hline insgesamt & 50 & 50 & 50 & 50 & 200 \\
\hline \hline
\end{tabular}

Tabelle 2.6: Unterschiedliche Szenarien der Berufsaufteilungen

Im Folgenden sei $k$ eine ordinal skalierte Variable mit den Ausprägungen $1,2, ., K$ für unterschiedliche nach beruflichem Status geordnete Berufsgruppen und $m$ bezeichne ungeordnete Kategorien 1,2,.,$M$ von Arbeitnehmern. Es wird angenommen, dass auf dem Arbeitsmarkt $T$ Arbeitskräfte beschäftigt sind, wobei jedes Individuum mit $m$ und $t$ klassifiziert wird. Die Ausdrücke $t_{m} ., t_{\cdot k}, t_{m k}$ geben die Anzahl der Arbeiter in Kategorie $m$, Kategorie $k$ und beiden Kategorien an. Innerhalb jeder ungeordneten Kategorie $m$ sei $c_{m k}$ das kumulierte Verhältnis 


$$
c_{m k}=\sum_{j=1}^{k} \frac{t_{m j}}{t_{m}} .
$$

Die Verteilung der Individuen in Kategorie $m$ auf die Berufsgruppen kann nun als $K$ Tupel $\boldsymbol{c}_{\boldsymbol{m}}=\left(c_{m 1}, c_{m 2}, \ldots, c_{m K}\right)$ dargestellt werden. Da $c_{m K}=1$ wird nur das [K-1]-Tupel $\boldsymbol{c}_{\boldsymbol{m}}=\left(c_{m 1}, c_{m 2}, \ldots, c_{m[K-1]}\right)$ benötigt, um die Verteilung in $m$ vollständig zu beschreiben. Auf dieselbe Weise wird die Verteilung sämtlicher Arbeiter auf die Berufsgruppen als $[K-1]$-Tupel $\boldsymbol{c}=\left(c_{1}, c_{2}, \ldots, c_{[K-1]}\right)$ dargestellt. Zusätzlich wird folgende Ausdrucksweise gewählt: Die Verteilung in $m$ dominiert die Verteilung in $n$ über die geordneten Berufsgruppen von $h$ bis $j(1 \leq h \leq j \leq K)$, wenn $c_{m k}<c_{n k}$ für alle $k \in(h, \ldots, j)$.

Folgende Eigenschaften sind bei der Messung ordinaler Segregation wünschenswert:

- Skaleninterpretierbarkeit: Ein Segregationsindex wird genau dann maximiert, wenn in jeder ungeordneten Kategorie $m$ die Arbeitskräfte nur einer Berufsgruppe zugehören $\left(c_{m k} \in\{0,1\}\right.$ für alle $m$ und $\left.k\right)$. Ein Segregationsindex wird genau dann minimiert, wenn in jeder ungeordneten Kategorie $m$ die Verteilung der einzelnen Arbeitskräfte auf die Berufsgruppen identisch ist mit der Verteilung sämtlicher Erwerbstätigen auf die Berufsgruppen $\left(c_{m k}=c_{k}\right.$ für alle $m$ und $\left.k\right)$. Die Intuition ist, dass maximale Segregation dann auftritt, wenn keine Variation in den geordneten Kategorien innerhalb jeder ungeordneten Gruppe auftritt und minimale Segregation dann, wenn die Verteilung von $k$ innerhalb $m$ der Verteilung sämtlicher Erwerbstätigen gleicht.

- Ungeordnete Kategorienäquivalenz: Wenn sich in zwei ungeordneten Kategorien $m$ und $n$ die Arbeitskräfte im selben Verhältnis auf die Berufsgruppen $k$ aufteilen und $m$ und $n$ zu einer Kategorie zusammengefasst werden, bleibt die Segregation unverändert. Absicht hierbei ist, dass zwei identisch verteilte ungeordete Kategorien nicht voneinander segregieren und in sofern auch der Zusammenschluss dieser Kategorien das Ausmaß der Segregation nicht beinflussen darf.

- Größeninvarianz: Wenn die Anzahl der Erwerbstätigen in jeder ungeordneten Kategorie $m$ und jeder geordeten Berufsgruppe $k$ mit derselben positiven Konstanten multipliziert wird $\left(c \cdot t_{m k}\right.$ für alle $m$ und $\left.k\right)$, bleibt die Segregation unverändert. Die Intuition hierbei ist, dass nur die Verteilung zwischen Kategorien und nicht die Anzahl der Arbeitskräfte insgesamt die Segregation beeinflusst.

- Nullgruppeneffekte: Wenn eine geordnete Kategorie $j$ ohne Arbeitskräfte $\left(t_{m j}=\right.$

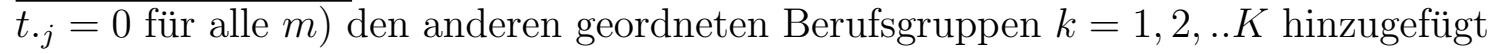
wird, sollen folgende Bedingungen erfüllt sein: 1 . Ist $j=0$ (d.h., Berufsgruppe $j$ hat geringeren Status als sämtliche $K$ Berufsgruppen) oder ist $j=K+1$ (d.h., Berufsgruppe $j$ hat höheren Status als sämtliche $K$ Berufsgruppen) bleibt die Segregation unverändert. 2. Ist $1<j<K$ und $c_{m j} \in\{0,1\}$ für alle $m$ (d.h, dass für 
sämtliche Arbeitskräfte in jeder ungeordneten Kategorie $m k<j$ oder $k>j$ gilt) und existieren ungeordnete Kategorien $m$ und $n$ mit $c_{m j}=0$ und $c_{n j}=1$ nimmt die Segregation zu, es sei denn sie hat schon ihren maximalen Wert erreicht. Die Absicht ist hierbei, dass sich Segregation durch das Hinzugügen von Nullkategorien nur dann ändern soll, wenn sich auch der Abstand zwischen den Berufsgruppen verändert.

- Wechsel: Wenn die Verteilung in $m$ die Verteilung in $n$ über die geordneten Berufsgruppen von $j$ bis $k$ dominiert und wenn ein Individuum aus Kategorie $j$ von $n$ nach $m$ wechselt während ein Individuum aus Kategorie $k$ von $m$ nach $n$ wechselt, nimmt die Segregation ab. Auf dieselbe Weise wird das Ausmaß der Segregation geringer, wenn ein Individuum aus Kategorie $m$ von $k$ nach $j$ wechselt während ein Individuum aus Kategorie $n$ von $j$ nach $k$ wechselt. Die Intuition ist hierbei, dass bei einem Wechsel von Individuen aus unterschiedlichen geordneten Kategorien in der Art, dass sich die Verteilungen in zwei ungeordneten Kategorien angleichen, die Segregation geringer wird.

- geordnete Wechsel: Wenn die Verteilung in $m$ die Verteilung in $n$ über die geordneten Berufsgruppen von $j$ bis $l$ dominiert (mit $j<k<l$ ) und wenn ein Individuum aus Kategorie $j$ von $n$ nach $m$ wechselt und gleichzeitig ein Individuum aus Kategorie $l$ von $m$ nach $n$ wechselt, dann ist das Ausmaß der Segregationsreduktion größer als wenn ein Individuum aus Kategorie $j$ von $n$ nach $m$ wechselt während ein Individuum aus Kategorie $k$ von $m$ nach $n$ wechselt. Gleichermaßen ist der Rückgang der Segregation größer, wenn ein Individuum aus Kategorie $m$ von $l$ nach $j$ wechselt während ein Individuum aus Kategorie $n$ von $j$ nach $l$ wechselt als wenn wenn ein Individuum aus Kategorie $m$ von $k$ nach $j$ wechselt während ein Individuum aus Kategorie $n$ von $j$ nach $k$ wechselt. Die Absicht hierbei ist, dass Wechsel zwischen Individuen aus weiter entfernten Kategorien die Segregation stärker beeinflussen als Wechsel zwischen Individuen aus näher gelegenen Kategorien.

- Additive ungeordnete Gruppenzerlegung: Wenn $M$ ungeordnete Kategorien in $N$ Cluster zusammengefasst werden, wird die Segregation wie in Reardon und Firebaugh (2002) beschrieben in unabhängige Komponenten innerhalb und zwischen den Clustern zerlegt.

- Additive geordnete Gruppenzerlegung: Wenn $K$ geordnete Kategorien in $J$ Superkategorien zusammengefasst werden durch den Zusammenschluss benachbarter Gruppen, soll die Segregation in eine Summe unabhängiger Komponenten innerhalb und zwischen den Superkategorien zerlegt werden.

Reardon und Firebaugh (2002) folgend wird ein ordinales Segregationsmaß $\Lambda^{v}$ wie folgt definiert:

$$
\Lambda^{v}=\sum_{m=1}^{M} \frac{t_{m}}{T v}\left(v-v_{m}\right),
$$


wobei $v$ die Streuung der ordinalen Variablen $k$ und $v_{m}$ die Streuung der ordinalen Variablen $k$ innerhalb Kategorie $m$ beschreibt 8 . Um ordinale Segregation zu bestimmen, wird für die Streuung einer ordinal skalierten Variablen mit der Ausprägung $k=1,2, \ldots, K$ ein Maximum $\boldsymbol{c}_{\mathbf{0}}$ definiert, wenn $k=1$ für die eine Hälfte der Arbeitskräfte und $k=K$ für die andere Hälfte, also $\boldsymbol{c}=\boldsymbol{c}_{\mathbf{0}}=\left(c_{1}, c_{2}, \ldots, c_{[K-1]}\right)=(1 / 2,1 / 2, \ldots, 1 / 2)$. Ein Minimum wird definiert, wenn $k=c$ mit $c \in(1,2, \ldots, K)$ für alle Beobachtungen . In diesem Fall existieren $K$ mögliche Verteilungen von $k$, wie z.B $\boldsymbol{c}=(0,0, \ldots, 0,1,1, \ldots 1)\left(c_{j}=0\right.$ für $j<k$ und $c_{j}=1$ für alle $j \geq k$ ). Messung der ordinalen Streuung läuft dann darauf hinaus, wie weit die Verteilung der ordinalen Variablen vom Maximum oder Minimum entfernt ist, wobei das Maximum zu 1 und das Minimum zu 0 normiert werden kann. Reardon (2008) schlägt vor, $\boldsymbol{c}$ als Verteilungsfunktion einer ordinalen Variablen zu betrachten, wobei die Streuung als Funktion des Abstandes zwischen $\boldsymbol{c}$ und der Geraden $c_{K}=1 / 2$ gemessen werden kann. Eine allgemeine Formulierung für das Streuungsmaß lautet:

$$
v=\frac{1}{K-1} \sum_{j=1}^{K-1} f\left(c_{j}\right)
$$

wobei $f(c)$ als stetige Funktion auf dem Intervall [0,1] definiert wird in der Form, dass $f(c)$ monoton wachsend für $c \in\left[0, \frac{1}{2}\right]$ und fallend für $\left.c \in\left(\frac{1}{2}, 1\right)\right]$ ist mit einem Maximalwert $f(1 / 2)=1$ auf dem Intervall [0,1] und einem Minimalwert $f(0)=f(1)=0$ auf dem Intervall $[0,1]$. Reardon (2008) nennt vier mögliche Funktionen $f \sqrt{9}$

$$
\begin{aligned}
& f_{1}(c)=-\left[c \log _{2} c+(1-c) \log _{2}(1-c)\right] \\
& f_{2}(c)=4 c(1-c) \\
& f_{3}(c)=2 \sqrt{c(1-c)} \\
& f_{4}(c)=1-|2 c-1|
\end{aligned}
$$

Werden diese Funktionen in Gleichung (2.34) eingesetzt, ergeben sich vier mögliche Maße für die Sreuung einer ordinalen Variablen, die entsprechend mit $v_{1}, v_{2}, v_{3}$ und $v_{4}$ bezeichnet werden. Sie nehmen alle den Wert 1 bei maximaler Streuung und den Wert 0 bei minimaler Streuung an. Gemäß Gleichung (2.33) erhält man vier Indizes zur Messung von Segregation:

\footnotetext{
${ }^{8}$ Wird $k$ als intervallskalierte Variable behandelt und ist $v$ die Varianz dieser Variablen, so ist $\Lambda^{v}$ äquivalent zu $R^{2}$, dem Bestimmtheitsmaß einer Regression von $k$ auf eine Menge von Dummy-Variablen für die Kategorien $m=1,2, \ldots, M$

${ }^{9}$ Reardon (2008) definiert $0 \cdot \log _{2}(0)=\lim _{x \rightarrow 0} x \log _{2} x=0$
} 


$$
\begin{aligned}
\Lambda^{1} & =\sum_{m=1}^{M} \frac{t_{m}}{T v_{1}}\left(v_{1}-v_{1 m}\right) \\
\Lambda^{2} & =\sum_{m=1}^{M} \frac{t_{m}}{T v_{2}}\left(v_{2}-v_{2 m}\right) \\
\Lambda^{3} & =\sum_{m=1}^{M} \frac{t_{m}}{T v_{3}}\left(v_{3}-v_{3 m}\right) \\
\Lambda^{4} & =\sum_{m=1}^{M} \frac{t_{m}}{T v_{4}}\left(v_{4}-v_{4 m}\right)
\end{aligned}
$$

Der Index $\Lambda^{1}$ ist eine ordinale Verallgemeinerung des konventionellen Zweigruppen- Informationstheorieindizes $H$ (Theil (1972), Theil und Finezza (1971), Zoloth (1976)) und soll im Folgenden $H^{0}$ bezeichnet werden. Bei zwei Berufsgruppen $(K=2)$ entsprechen $H^{0}$ und $H$. Auf ähnliche Art entspricht $\Lambda^{2}$ einer ordinalen Verallgemeinerung des Variationsratioindizes (James und Taeuber (1985), Reardon und Firebaugh (2002)) und soll im Folgenden $R^{0}$ bezeichnet werden. Für $K=2$ entsprechen $R^{0}$ und $R$. Des Weiteren kann $\Lambda^{3}$ als ordinale Verallgemeinerung des Hutchens-Square-Root-Indizes $S$ (Hutchens (2004)) angesehen werden und wird im Folgenden mit $S^{0}$ bezeichnet. Auch hier entsprechen für $K=2$ die Indizes $S^{0}$ und $S$. Im Gegensatz zu den vorher beschriebenen Indizes ist $\Lambda^{4}$ nicht vergleichbar mit anderen Segregationsmaßen. Die vier Indizes können als durchschnittliche Distanz zwischen der Geamtstreuung einer ordinalen Variablen und ordinaler Streuung innerhalb kategorialer Gruppen betrachtet werden. Sie unterscheiden sich nur in der funktionalen Form, wie die Streuung gemessen wird. Reardon (2008) zeigt, dass die meisten zuvor beschriebenen Eigenschaften, die bei der Messung von Segregation wünschenswert sind, von den Indizes erfüllt werden. Die Eigenschaften Skaleninterpretierbarkeit und Wechsel werden nicht von $\Lambda^{4}$ und additive geordnete Gruppenzerlegung wird von keinem der aufgeführten Segregationsmaße erfüllt.

\subsubsection{Empirische Resultate}

In der folgenden Studie wird das Verfahren nach Reardon (2006) zunächst angewandt, um berufliche Segregation zwischen Männern und Frauen und anschließend zwischen Ausländern, Ausländerinnen, Inländern und Inländerinnen zu ermitteln. Die Stichproben entsprechen den schon in Kapitel 2 verwendeten und umfassen für das Jahr 2006 3012 Inländer, 258 Ausländer, 2974 Inländerinnen und 192 Ausländerinnen, für das Jahr 19991748 Inländer, 231 Ausländer, 1065 Inländerinnen und 71 Ausländerinnen. Die Berufsgruppen unterscheiden erneut nach beruflichem Status, ansteigend von Kategorie eins bis fünf. 
Tabelle (2.7) zeigt, wie sich die vier ungeordneten Kategorien von Arbeitnehmern auf die Berufsgruppen aufteilen,

\begin{tabular}{rcccccr}
\hline \hline & & & & & & \\
Jahr & Gruppe & Inländer & Ausländer & Inländerinnen & Ausländerinnen & $\sum$ \\
\hline \multirow{2}{*}{2006} & 1 & 404 & 118 & 306 & 87 & 915 \\
& 2 & 1160 & 97 & 778 & 57 & 2092 \\
& 3 & 811 & 34 & 1591 & 46 & 2482 \\
& 4 & 309 & 5 & 180 & 1 & 495 \\
& 5 & 0328 & 4 & 119 & 1 & 452 \\
& $\sum$ & 3012 & 258 & 2974 & 192 & 6436 \\
\hline \hline & & & & & & \\
1999 & 1 & 219 & 116 & 112 & 37 & 484 \\
& 2 & 569 & 76 & 279 & 17 & 941 \\
& 3 & 399 & 18 & 511 & 15 & 943 \\
& 4 & 492 & 19 & 146 & 2 & 659 \\
& 5 & 69 & 2 & 17 & 0 & 88 \\
& $\sum$ & 1748 & 231 & 1065 & 71 & 3115 \\
\hline \hline
\end{tabular}

Tabelle 2.7: Berufsaufteilung

Tabelle (2.8) die Ergebnisse der Segregationsmessungen.

\begin{tabular}{cccccc}
\hline \hline \multirow{2}{*}{ Jahr } & Gruppen & $\Lambda^{1}$ & $\Lambda^{2}$ & $\Lambda^{3}$ & $\Lambda^{4}$ \\
\hline \multirow{2}{*}{2006} & Männer, Frauen & 0.018 & 0.018 & 0.016 & 0.054 \\
& Inländer/,-innen & 0.046 & 0.048 & 0.038 & 0.072 \\
& Ausländer/,-innen & & & & \\
\hline \hline \multirow{2}{*}{1999} & Männer, Frauen & 0.013 & 0.014 & 0.010 & 0.000 \\
& Inländer/,-innen & 0.056 & 0.064 & 0.044 & 0.071 \\
& Ausländer/,-innen & & & & \\
\hline \hline
\end{tabular}

Tabelle 2.8: Ergebnisse der Segregationsmessungen

Die Segregation zwischen Männern und Frauen fällt bei der Methode nach Reardon (2006) geringer aus als bei den in Kapitel 2 verwendeten, die Berufsgruppen kategorial behandelnden Indizes, wobei die Ungleichverteilung auf die Berufe zwischen 1999 und 2006 geringfügig angestiegen ist. Wird zusätzlich zwischen In- und Ausländern unterschieden erhöht sich das Ausmaß der Segregation im betrachteten Zeitraum. 


\subsection{Resüme}

In dieser Studie wurden zunächst traditionelle Methoden angewandt, um zu messen, wie sich berufliche Segregation zwischen männlichen und weiblichen Arbeitnehmern im Zeitraum von 1999 - 2006 entwickelt hat. Mit Ausnahme des $S R$ nehmen sämtliche vorgestellten Indizes den Wert 0 bei nicht vorhandener Segregation und den Wert 1 bei vollständiger Segregation an. Die vorgestellten traditionellen Messverfahren sind nur für den Zweigruppen- und nicht für den Mehrgruppenfall geeignet. Wird der Ginikoeffizient $G$ als Maßzahl herangezogen, so fällt die Segregation zu beiden Zeitpunkten mit 0.291 (2006) und 0.298 (1999) am Größten, beim Square-Root-Index $S$ mit 0.039 (2006) und 0.041 (1999) am Geringsten aus. Die Werte des Sex Ratios können nicht mit den anderen Indizes verglichen werden, da es sich hierbei um ein nicht normiertes Maß handelt. Die Indexwerte bleiben im Zeitverlauf relativ konstant. Während ID und IP zu dem Ergebnis kommen, dass die Segregation im Zeitverlauf geringfügig zugenommen hat, bleiben die anderen Maßahlen konstant oder gehen geringfügig zurück.

Eine weitere Möglichkeit zur Messung von Segregation besteht in der Anwendung des Mutual-Information-Indizes $M$, der sich auf den Informationstheorieindex $\mathrm{H}$ bezieht. Mit Hilfe des von Frankel und Volij (2008) entwickelten Indizes $M$ kann gemessen werden, wie gleich oder ungleich sich ethnische Gruppen auf Schulen über unterschiedliche Regionen verteilen. Der Vorteil des Mutual-Information-Indizes gegenüber anderen Messverfahren besteht darin, dass er eine Vielzahl wünschenswerter Eigenschaften erfüllt. Die in dieser Studie betrachteten Schulegionen sind die Samtgemeinden Nenndorf und Rodenberg im Landkreis Schaumburg in Niedersachsen. Bei den Schulen wurden Grundschulen ausgewählt, da sich diese im Gegensatz zu anderen Schulformen in beiden Gemeinden befinden, bei den ethnischen Gruppen wird zwischen In- und Ausländern unterschieden. Die Segregation fällt in beiden Gemeinden gering aus. Der Mutual-Information-Index nimmt für die Samtgemeinde Nenndorf den Wert 0.020 und für die Samtgemeinde Rodenberg den Wert 0.001 an. Die Messung der Segregation für den Zusammenschluss beider Gemeinden in einer Region kann nach Gleichung (2.17) oder (2.21) erfolgen und ist gleich 0.010. Wird der Informationstheorieindex zur Messung der Segregation herangezogen ist das Ergebnis vergleichbar.

Kim und Jargowsky (2005) entwickeln eine modifzierte Form des Gini-Koeffizienten G, der auch als Segregationsmaß für stetige Variablen angewandt werden kann. In dieser Studie wurde gemessen, wie gleich oder ungleich sich das jährliche Nettohaushaltseinkommen auf die Regionen Nord, Süd, West und Ost verteilt. Der Gini-Koeffizient $G_{i}$ für die Einkommen der individuellen Haushalte steigt im betrachteten Zeitraum von 0.324 auf 0.373, der Gini-Koeffizient $G_{n}$ für die mittleren Einkommen der Haushalte in den Regionen von 0.037 auf 0.054 und der Segregationsindex $G_{S}$ von 0.114 auf 0.145 .

Traditionelle Methoden zur Messung von Segregation beschreiben, wie sich nominal skalierte Gruppen (z.B. Männer und Frauen) auf ebenfalls nominal skalierte organisatorische 
Einheiten (z.B. Regionen, Schulen) verteilen. Reardon (2008) entwickelt einen allgemeinen Ansatz, mit dem Segregation gemessen werden kann, wenn entweder die Gruppen oder die organisatorischen Einheiten ordinal skaliert sind. In dieser Studie wurde zunächst berufliche Segregation zwischen Männern und Frauen und anschließend zwischen Ausländern, Ausländerinnen, Inländern und Inländerinnen ermittelt. Die Berufsgruppen unterscheiden nach beruflichem Status, ansteigend von Kategorie eins bis fünf. Die Segregation zwischen Männern und Frauen fällt bei der Methode nach Reardon (2006) geringer aus als bei den die Berufsgruppen kategorial behandelnden Indizes, wobei die Ungleichverteilung auf die Berufe zwischen 1999 und 2006 geringfügig angestiegen ist. Wird zusätzlich zwischen Inund Ausländern unterschieden, so erhöht sich das Ausmaß der Segregation im betrachteten Zeitraum.

\section{Literatur}

AnAnd, S. (1983) Inequality and Poverty in Malaysia: Measurement and Decomposition, Oxford: Oxford University Press.

Blackburn R.-M., Siltanen J.,Jarman J. (1995). The Measurement of Occupational Gender Segregation: Current Problems and a New Approach Journal of the Royal Statistical Society, Vol. 158, Part 2, S. 319-331.

Chakravarty, S-R., D’Ambrosio, C., Silber J. (2009). Generalized gini occupational segregation indices, in Yves Flückiger, Sean F. Reardon, Jacques Silber (ed.) Occupational and Residential Segregation, Research on Economic Inequality, Vol. 17, Emerald Group Publishing Limited, S.71-95.

Duncan, O.D., Duncan, B. (1955). A Methodological Analysis of Segregation Indices, American Sociological Review, Vol. 20, No. 2, S. 210-217.

FALK, S. (2002). Geschlechtsspezifische berufliche Segregation in Ostdeutschland zwischen Persistenz, Verdrängung und Angleichung: ein Vergleich mit Westdeutschland für die Jahre 1991-20, Mitteilungen aus der Arbeitsmarkt- und Berufsforschung, Vol. 35, Issue 1, S. 37-59.

Frankel, D.-M., VoliJ, O. (2008). Measuring school segregation, Discussion Paper, No. 07-03, Monaster Center for Economic Research, Ben Gurion University of the Negev.

Fuchs, V. (1975). A Note on Sex Segregation in Professional Occupations, Explorations in Economic Research, Vol. 2, S. 105-111.

GibBs, J. (1965). Occupational Differentiation of Negroes and Whites Social Forces, Vol. 44, No 2, S. 59-165.

Gross, E. (1968). Plus ça change...? The Sexual Structure of Occupations Over Time, Social Problems, Vol. 16, No 2, S. 198-208. 
Hakim, C. (1981). Job Segregation: Trends in the 1970s, Employment Gazette, December, S. 521- 529 .

Hutchens, R.-M. (2001). Numerical measures of segregation: Desirable properties and their implications. Mathematical Social Sciences, Vol 42, No 1, S. 13-29.

Hutchens, R.-M. (2004). One measure of segregation International Economic Review, Vol. 45 , No. 2), S. 555-578.

Jahn,J., Schmid, C.-F., Schrag, C. (1947). The Measurement of ecological segregation, American Sociological Review, Vol. 3, S. 293-303.

James, D. R., TAeuber, K. E. (1985). Measures of segregation, Sociological Methodology, Vol. 14, S. 1-32.

Karmel, T. And Maclachlan, M. (1988). Occupational Sex Segregation - Increasing or Decreasing, Economic Record, Vol. 64, S. 187-95.

Kim, J., Jargowsky, P.-A. (2005). The Gini Coefficient and Segregation on a Continuous Variable National Poverty Center Working Paper Series, No. 05-02, Gerald R. Ford School of Public Policy, University of Michigan.

Melly, B. (2005). Public-private sector wage differentials in Germany: Evidence from quantile regression, Empirical Economics, Vol. 30, No 2, S. 505-520.

Mora, R., Ruiz-Castillo, J. (2003). Additively Decomposable Segregation Indexes. The Case of Gender Segregation by Occupations and Human Capital Levels in Spain, Journal of Economic Inequality, Springer, vol. 1, No. 2, S. 147-179.

Mora, R., Ruiz-Castillo, J. (2004). Gender segregation by occupations in the public and the private sector. The case of Spain, Investigaciones Economicas, Fundación SEPI, vol. 28, No 3, S. 399-428.

Mora, R., Ruiz-Castillo, J. (2007). The invariance properties of the Mutual Information index of multigroup segregation, Economics Working Papers, we077544, Universidad Carlos III, Departamento de Economía.

Reardon, S.F. (2008). Measures of Ordinal Segregation, Research on Economic Inequality, Working Paper, 2008-11.

Reardon, S.-F., Firebaugh, G. (2002). Measures of multi-group segregation. Sociological Methodology, Vol 32, S. 33-67.

Siltanen, J., Jarman, J. And Blackburn, R.-M. (1995). Gender Inequality in the Labour Market: Occupational Concentration and Segregation, Geneva: International Labour Office. 
Taeuber, A-F., Taeuber, K.-E. (1965). Negroes in Cities: Residential Segregation and Neighborhood Change Chicago: Aldine Publishing Company.

TheIL, H. (1972). Statistical decomposition analysis, Amsterdam: North Holland Publishing Company, Vol. 14.

Theil, H., FinezzA, A. J. (1971). A note on the measurement of racial integration of schools by means of informational concepts, Journal of Mathematical Sociology, Vol. 14, S. 187-194.

Treiman, D. J. (1977). Occupational prestige in comparative perspective, Academic Press, New York.

Zoloth, B. S. (1976). Alternative measures of school segregation ,Land Economics, Vol. 52 No.3, S. 278-298. 


\section{Kapitel 3}

\section{Ökonometrische Methoden zur Messung von Lohndiskriminierung}

\subsection{Einleitung}

Die Gleichstellung von Männern und Frauen stellt in der Sozialpolitik der Europäischen Union eine der grundlegenden Aufgaben dar. Bereits in den 70er Jahren wurden in der Europäischen Gemeinschaft verbindliche Richtlinien zur Umsetzung der sozialpolitischen Gleichstellung der Geschlechter erlassen. Mit Inkrafttreten des Anti-Diskriminierungsgesetzes (ADG) am 18. August 2006 wurden mehrere EU-Richtlinien in deutsches Recht umgesetzt. Ziel des Gesetzes ist, Benachteiligungen aus Gründen der Rasse, der ethnischen Herkunft, des Geschlechts, der Religion oder Weltanschauung, einer Behinderung, des Alters oder der sexuellen Identität zu verhindern oder zu beseitigen ( $§ 1$ ADG). Neben einigen anderen Bereichen wird durch dieses Gesetz vor allem das Arbeitsrecht berührt. Benachteiligte Arbeitnehmer haben Anspruch auf Ersatz des ihnen entstanden materiellen und immateriellen Schadens. Es ist daher von großem Interesse, inwieweit unterschiedliche Löhne zwischen Gruppen auf Diskriminierung zurückzuführen sind. Hierfür stehen verschiedene Messerfahren zur Verfügung.

Die dominierende Theorie zur Erklärung von Einkommensunterschieden ist die Humankapitaltheorie (Becker 1964, Mincer 1974). Zentrale Hypothese dieser Theorie ist, dass Arbeitnehmer in ihr Humankapital investieren, um ihre Produktivität und damit ihren Lohn zu steigern. Als Humankapital gelten das über das Schul- und Ausbildungssystem erworbene Wissen, aber auch die über die Berufstätigkeit angeeigneten Qualifikationen. Als Ausgangspunkt von empirischen Studien dient die humankapitaltheoretische Lohnfunktion von Mincer aus dem Jahr 1974. Sie spezifiziert einen semilogarithmischen Zusammenhang zwischen dem Einkommen als abhängiger Variable und Einflussgrößen, die Determinanten des allgemeinen Humankapitals darstellen und das Produktionsergebnis beeinflussen. Lohndiskriminierung liegt dann vor, wenn Gruppen bei gleichen produktivitätsrelevanten Merkmalen unterschiedlich entlohnt werden. 
Becker $(1957,1971)$ erklärt die schlechtere Entlohnung von Frauen dadurch, dass Unternehmer eine Neigung zur Diskriminierung ("taste of discrimination") gegenüber dieser Personengruppe entwickeln. Ihnen entstehen bei der Zusammenarbeit mit Frauen psychische, nicht-monetäre Kosten, die sich mittels eines Diskriminierungskoeffizienten ausdrücken lassen und den Nutzen der Unternehmer verringern. Statistische Diskriminierung kann nach Phelps (1972), Arrow (1973) und Spence (1973) resultieren, wenn bei Stellenbesetzungen Gruppenmerkmale zur Schätzung der Produktivität der Bewerber herangezogen werden. Gelten Männer als durchschnittlich produktiver als Frauen, werden Angehörige der diskriminierten Gruppe gar nicht oder nur zu einem geringeren Lohn eingestellt, der die Differenz der Produktivitätserwartungen ausgleicht. Opfer der statistischen Diskriminierung sind dabei die Frauen, deren Produktivität über dem Gruppenmittelwert liegt. Nach Bergmann (1974) und Sorensen (1990) resultiert Diskriminierung aus dem für Frauen stark eingeschränkten Zugang zu Männerberufen. Aufgrund der dadurch bedingten Überfüllung ("crowding") der weiblichen Teilarbeitsmärkte können Frauen mit Lohnsätzen unter dem Gleichgewichtslohn der Männer beschäftigt werden.

Die ersten bedeutenden Studien auf dem Gebiet der Lohndiskriminierungsmessungen sind von Oaxaca (1973) und Blinder (1973), im Folgenden OB bezeichnet, veröffentlicht worden. Sie untersuchen, inwieweit die Lohnlücke zwischen Männern und Frauen sowie schwarzen und weißen Arbeitnehmern am US-amerikanischen Arbeitsmarkt auf Diskriminierung zurückzuführen ist. OB schlagen vor, eine Regressionsschätzung für Männer und Frauen getrennt durchzuführen. Mithilfe der aus den Lohnregressionen geschätzten Koeffizienten, welche die Entlohnungen der Humankapitalcharakteristika widerspiegeln, wird die gewichtete Differenz in den mittleren Humankapitalausstattungen zwischen den beiden Gruppen ermittelt. Diese wird dann als erklärter Teil des Lohnunterschiedes bezeichnet. Der verbleibende unerklärte Teil der Lohnlücke wird als Maß für Diskriminierung herangezogen. Ein Kritikpunkt am Ansatz nach OB besteht darin, dass entweder ausschließlich die Lohnstruktur der Männer oder die der Frauen als die nicht diskriminierte zugrunde gelegt wird, was zu unterschiedlichen Ergebnissen bei der Messung von Lohndiskriminierung führt. Alternativ wird vorgeschlagen, sowohl die Lohnstruktur der Männer als auch die der Frauen zu verwenden und zusätzlich einen gewichteten Parametervektor für die Lohnstruktur in Abwesenheit von Diskriminierung einzuführen. Bei Reimers (1983) gehen bei der Gewichtung männliche und weibliche Lohnstruktur im selben Verhältnis ein, bei Cotton (1988) hängt die Gewichtung vom Anteil der Männer in der Stichprobe ab. Neumark (1988) zieht als Basis der Gewichtung die gepoolte Stichprobe heran, Oaxaca und Ransom (1994) schlagen eine allgemeine Form der Gewichtung vor, bei der die Gewichtungsmatritzen von Reimers und Cotton Spezialfälle darstellen.

Brown, Moon und Zoloth (1980) zerlegen die Lohnlücke auf die gesamte Verteilung der Berufe und berücksichtigen somit, dass Frauen in größerem Maße schlechter bezahlte Berufe ausüben als es den Unterschieden in der Leistungsfähigkeit und Leistungswilligkeit entspricht. Dolton und Kidd (1994) sowie Miller (1987) zeigen, dass weit mehr als die Hälfte des unkorrigierten Lohndifferentials auf Lohnunterschiede innerhalb der Berufe 
und nicht auf eine unterschiedliche Verteilung von Männern und Frauen auf diese Berufe zurückzuführen ist. Bayard, Hellerstein, Neumark und Troske (2003) sowie Gupta und Rothstein (2001) weisen nach, dass der Lohn in Berufsgruppen innerhalb von Betrieben (Jobzellen) umso geringer ausfällt, je höher der Frauenanteil ist. Appleton, Hoddinott und Krishnan (1999) liefern eine Erweiterung des Ansatzes nach Brown, Moon und Zoloth (1980), indem sie zur Bestimmung der Verteilung von Arbeitnehmern auf die Berufe in Abwesenheit von Diskriminierung nicht nur die Verteilung von Männern sondern auch von Frauen heranziehen.

Juhn, Murphy und Pierce (1991) entwickeln einen Ansatz, der in empirischen Studien angewandt wird, um den Lohnunterschied im Zeitverlauf oder zwischen Ländern zu untersuchen. Sie führen die Lohnlücke auf Unterschiede in den beobachtbaren Humankapitalcharakteristika und auf unbeobachtete individuelle Fähigkeiten zurück, die sich in unterschiedlichen Residuen niederschlagen. Sie unterstellen für Männer und Frauen dieselben Ertragsraten und ziehen für die weitere Analyse die Lohnstruktur der Männer heran. Suen (1997) kritisiert die Interpretation dieses Ansatzes und zeigt, dass Arbeitsmarktdiskriminierung zu einer Veränderung der relativen Position der Frauen führen kann, obwohl sich weder das Ausmaß der Diskriminierung noch der relative Grad der unbeobachteten Fähigkeiten geändert hat. Blau und Kahn (2006) wenden den Dekompositionsansatz nach Juhn, Murphy und Pierce (1991) zum einen mit der Lohnstruktur der Männer und zum anderen mit der Lohnstruktur der Frauen an und kommen dabei zu ähnlichen Ergebnissen.

Die bisher vorgestellten, auf OLS-Schätzungen beruhenden Methoden zur Messung von Lohndiskriminierung, untersuchen, inwieweit unterschiedliche mittlere Löhne zwischen zwei Gruppen mit speziellen Merkmalsausprägungen auf Diskriminierung zurückzuführen sind. Hauptnachteil dieser Verfahren besteht darin, dass Einkommensunterschiede innerhalb der Gruppen unberücksichtigt bleiben. Es ist aber durchaus von Interesse, inwieweit Lohndiskriminierung beispielsweise zwischen weiblichen und männlichen Spitzen- oder Geringverdienern vorliegt. Die Verfahren nach DiNardo, Fortin und Lemieux (1996) sowie nach Machado und Mata (2005), Melly (2005) ermöglichen dagegen die Dekomposition des unkorrigierten Lohndifferentials nicht nur für die mittleren Löhne, sondern für sämtliche Quantile der Lohnverteilung. Eine solche Zerlegung bereitet insofern Schwierigkeiten, da zwar die Verteilungen der Löhne von Männern und Frauen beobachtbar sind, nicht aber die kontrafaktischen Lohnverteilungen, d.h. die Verteilung der Löhne von Männern mit Humankapitaleigenschaften von Männern und Lohnstruktur der Frauen bzw. die Verteilung der Löhne von Frauen mit Humankapitaleigenschaften von Frauen und Lohnstruktur der Männer. Beim Dekompositionsansatz nach DiNardo, Fortin und Lemieux (1996) werden die kontrafaktischen Lohnverteilungen mithilfe von gewichteten Kerndichteschätzungen ermittelt. Machado und Mata (2005) kombinieren bei ihrem Ansatz das Instrumentarium der von Koenker und Bassett (1978) eingeführten Quantilsregressionen mit einem Bootstrap-Ansatz. Während Machato und Mata (2005) die Veränderungen der Lohnverteilung im Zeitverlauf analysieren, schlägt Melly (2005) eine Dekomposition der Lohnlücke vor, die einen Vergleich mit dem Ansatz nach OB vereinfacht. 
Der Dekompositionsansatz nach Nopo (2008) stellt eine nicht parametrische Alternative des Verfahrens nach OB dar und benötigt keine Schätzung der Lohnverteilungen. Es wird zwischen Individuen unterschiedlicher Gruppen, deren Humankapitalcharakteristika sich nicht unterscheiden ("common support") und Individuen außerhalb dieses Bereiches ("out-of-support") unterschieden. Sämtliche Beobachtungen werden in der weiteren Analyse mit einbezogen. Das Lohndifferential wird in vier Terme zerlegt, wobei zwei denjenigen des Ansatzes nach OB entsprechen.

Ziel dieses Beitrags ist, die gängigen Methoden zur Messung von Lohndiskriminierung vorzustellen, zu vergleichen sowie Stärken und Schwächen der einzelnen Verfahren zu diskutieren. Methodische Ansätze sind ebenso Gegenstand der nachfolgenden Ausführungen wie die Darstellung empirischer Ergebnisse, die sich auf den deutschen Arbeitsmarkt konzentrieren. Dieser Artikel ist folgendermaßen gegliedert: In Abschnitt 2 wird der verwendete Datensatz und die Auswahl der Variablen erläutert, in den Abschnitten 3-7 werden die gängigen Dekompositionsverfahren vorgestellt, und in Abschnitt 8 erfolgt ein abschließendes Resüme. Bei sämtlichen vorgestellten Verfahren werden logarithmierte Löhne verwendet, um eine bessere Vergleichbarkeit sämtlicher Methoden zu ermöglichen.

\subsection{Die Daten}

Für die Anwendung der zuvor beschriebenen Methoden wird das vom Deutschen Institut für Wirtschaftsforschung (DIW) erstellte Sozio-ökonomische Panel (SOEP) verwendet. Es handelt sich hierbei um eine repräsentative Befragung privater Haushalte in Deutschland, die im jährlichen Rhythmus bei denselben Personen und Haushalten seit 1984 durchgeführt wird. Bereits im Juni 1990, also noch vor der Währungs-, Wirtschafts- und Sozialunion, wurde die Studie auf das Gebiet der ehemaligen DDR ausgeweitet.

Bei der den folgenden Untersuchungen zugrunde liegenden Stichproben sind sozialversicherungspflichtige Arbeiter, Angestellte und Beamte in Vollzeit- oder Teilzeittätigkeit von 1865 Jahren aus den zugehörigen SOEP-Wellen herausgefiltert worden. Nicht berücksichtigt sind Selbständige, Praktikanten und Auszubildende, unbezahlt mithelfende Familienangehörige und Personen, die ausschließlich in so genannten Mini-Jobs oder 1-Euro-Jobs tätig sind. Die in den Abschnitten 3-6 vorgestellten Studien beziehen sich auf das Jahr 2006 für Gesamtdeutschland. Bei Anwendung der in Kapitel 7 beschriebenen Methode nach Juhn, Murphy und Pierce (1991) wird die Lohnentwicklung im Zeitraum von 1989 bis 1993, also vor und nach der Deutschen Wiedervereinigung, in den alten Bundesländern herangezogen.

Folgende Variablen werden für die empirische Analyse aus dem SOEP-Datensatz mithilfe der SOEP-Info generiert: 
- Logwage: Logarithmierter Bruttotundenlohn. Sollen in Deutschland die Verdienstsituationen unterschiedlicher Arbeitnehmer oder Arbeitnehmergruppen verglichen werden, sollte man hierzu möglichst Jahresverdienstangaben verwenden, da diese - im Gegensatz zu Monats- oder Wochenverdiensten - auch die unregelmäßigen Zahlungen in Form von 13., 14. Monatsgehalt, Weihnachtsgeld, Urlaubsgeld, Gewinnbeteiligungen und Sondervergütungen beinhalten. Der Bruttostundenlohn wurde als Quotient aus dem Jahresbruttoverdienst (tatsächlicher Bruttoverdienst des letzten Monats multipliziert mit 12 zuzüglich erhaltener Extrazahlungen) und der tatsächlich geleisteten Arbeitszeit (tatsächlich gearbeitete Wochenstunden multipliziert mit 52) errechnet, wobei die wöchentliche Arbeitszeit auf 60 Wochenstunden beschränkt wird. Es wird dabei unterstellt, dass Personen mit höherer Wochenstundenzahl, diese durch Tätigkeiten außerhalb der normalen Berufsarbeitszeit erreichen wie z.B durch alle Tätigkeiten mit Geschäftskollegen. Arbeitnehmer mit einem Bruttostundenlohn von null Euro wurden ausgeschlossen. Gründe hierfür sind beispielsweise Insolvenz des Arbeitgebers oder ein gerade erst vollzogener Wechsel des Arbeitsplatzes und eine damit verbundene noch ausstehende erste Lohnzahlung.

- Edu: Bildungsjahre (7-18). Bei sämtlichen Verfahren wird im Zusammenhang mit der Darstellung des individuell erreichten Bildungsstands der Ansatz nach Bildungsjahren verwendet. Bildung wird ausgedrückt in Jahren, die institutionell für das Erreichen des höchsten gegebenen Bildungsabschlusses einer Person nötig sind. Ein erfolgreiches Universitätsstudium nach Gymnasialbesuch wird hier beispielsweise mit 18 Jahren bewertet (13 Jahre Schulausbildung +5 Jahre Universitätsausbildung). Personen ohne jeglichen Abschluss erhalten bei dieser Vorgehensweise 7 Ausbildungsjahre, was in etwa der Einhaltung der gesetzlichen Schulpflicht entspricht. Edu_2: Bildungsjahre quadriert.

- Ten,Ten_2 : Betriebszugehörigkeit in Jahren und in Jahren quadriert.

- Exp, Exp_2: Berufserfahrung in Jahren und in Jahren quadriert.

- Erljob: Dummyvariable für Tätigkeit im erlernten Beruf. Erljob = 1, wenn Tätigkeit im erlernten Beruf erfolgt.

- Le200, Le2000, G2000: Dummyvariablen für Betriebsgröße.

- Le20 = 1 bei Betrieben mit höchstens 20 Mitarbeitern.

- Le200 = 1 bei Betrieben mit mehr als 20 und höchstens 200 Mitarbeitern .

- Le2000 = 1 bei Betrieben mit mehr als 200 und höchstens 2000 Mitarbeitern.

- G2000 = 1 bei Betrieben mit mehr als 2000 Mitarbeitern .

- Ger: Dummyvariable für Nationalität. Ger = 1 bei Inländern. 
- West: Dummyvariable für Arbeitsort. West $=1$, wenn Tätigkeit in den alten Bundesländern erfolgt.

- VaAkad: Dummyvariable für Berufsbildung des Vaters. VaAkad = 1, wenn Vater mit Fachhochschul- oder Universitätsabschluss.

- VaoA: Dummyvariable für Berufsbildung des Vaters. VaoA = 1, wenn Vater ohne Ausbildung.

Arbeitnehmer mit fehlenden oder unplausiblen Antworten werden wie in vergleichbaren, auf dem SOEP basierenden Analysen (vgl. Melly (2006)) nicht berücksichtigt. Hierbei wird unterstellt, dass keine systematische Antwortverweigerung oder -verfälschung vorliegt. Fehlende Werte sind über sämtliche Beobachtungen zufällig verteilt und somit ist kein Selektionsbias zu erwarten.

\subsection{Auf Lohnregressionen basierende Methoden zur Messung von Lohndiskriminierung}

\subsubsection{Methodologie}

Als Ausgangspunkt des Ansatzes nach nach Oaxaca (1973) und Blinder (1973) dient die humankapitaltheoretische Lohnfunktion von Mincer:

$$
\begin{aligned}
\ln y_{M i} & =\boldsymbol{\beta}_{M}^{\prime} \boldsymbol{x}_{M i}+u_{M i}, \\
\ln y_{F i} & =\boldsymbol{\beta}_{F}^{\prime} \boldsymbol{x}_{F i}+u_{F i},
\end{aligned}
$$

wobei $\ln y_{M i}, \ln y_{F i}$ die logarithmierten Löhne und $\boldsymbol{\beta}_{M}, \boldsymbol{\beta}_{F}$ die zu schätzenden Parametervektoren (Intercept und Steigungsparameter) für Individuum $i$ darstellen. Die Vektoren $\boldsymbol{x}_{M i}, \boldsymbol{x}_{F i}$ beschreiben die beobachteten Qualifikationen der Individuen und enthalten zusätzlich sozioökonomische und regionale Komponenten. Das erste Element dieser Vektoren ist gleich eins, um den Intercept mitzuberücksichtigten. Die Ausdrücke $u_{M i}, u_{F i}$ stellen Störvariablen dar. Der Index $M$ kennzeichnet die einkommensstärkere, männliche Gruppe, der Index $F$ die einkommensschwächere, weibliche Gruppe. Im ersten Schritt werden die Regressionskoeffizienten geschätzt und für beide Geschlechter der mittlere logarithmierte Lohn ermittelt. Das Lohndifferential $\overline{\ln y_{M}}-\overline{\ln y_{F}}$ kann anschließend folgendermaßen zerlegt werden:

$$
\begin{aligned}
\overline{\ln y_{M}}-\overline{\ln y_{F}} & =\hat{\boldsymbol{\beta}}_{M}^{\prime} \overline{\boldsymbol{x}}_{M}-\hat{\boldsymbol{\beta}}_{F}^{\prime} \overline{\boldsymbol{x}}_{F} \\
& =\hat{\boldsymbol{\beta}}_{M}^{\prime} \overline{\boldsymbol{x}}_{M}+\hat{\boldsymbol{\beta}}_{M}^{\prime} \overline{\boldsymbol{x}}_{F}-\hat{\boldsymbol{\beta}}_{M}^{\prime} \overline{\boldsymbol{x}}_{F}-\hat{\boldsymbol{\beta}}_{F}^{\prime} \overline{\boldsymbol{x}}_{F} \\
& =\underbrace{\hat{\boldsymbol{\beta}}_{M}^{\prime}\left(\overline{\boldsymbol{x}}_{M}-\overline{\boldsymbol{x}}_{F}\right)}_{\text {Ausstattungseffekt }}+\underbrace{\left(\hat{\boldsymbol{\beta}}_{M}-\hat{\boldsymbol{\beta}}_{F}\right)^{\prime} \overline{\boldsymbol{x}}_{F}}_{\text {Diskriminierungseffekt }}
\end{aligned}
$$


bzw.

$$
\overline{\ln y_{M}}-\overline{\ln y_{F}}=\underbrace{\hat{\boldsymbol{\beta}}_{F}^{\prime}\left(\overline{\boldsymbol{x}}_{M}-\overline{\boldsymbol{x}}_{F}\right)}_{\text {Ausstattungseffekt }}+\underbrace{\left(\hat{\boldsymbol{\beta}}_{M}-\hat{\boldsymbol{\beta}}_{F}\right)^{\prime} \overline{\boldsymbol{x}}_{M}}_{\text {Diskriminierungseffekt }},
$$

wobei $\hat{\boldsymbol{\beta}}_{M}, \hat{\boldsymbol{\beta}}_{F}$ die geschätzten Parametervektoren der unterschiedlichen Lohnstrukturen bei Männern und Frauen darstellen.

Die Ausdrücke $\hat{\boldsymbol{\beta}}_{M}^{\prime}\left(\overline{\boldsymbol{x}}_{M}-\overline{\boldsymbol{x}}_{F}\right)$ bzw. $\hat{\boldsymbol{\beta}}_{F}^{\prime}\left(\overline{\boldsymbol{x}}_{M}-\overline{\boldsymbol{x}}_{F}\right)$ in Gleichung 3.2 und 3.3 stellen den erklärten Teil des Lohnunterschiedes dar und werden als Ausstattungseffekt bezeichnet. Dieser entspricht in Gleichung (3.2) dem hypothetischen Mindereinkommen der Männer und in Gleichung (3.3) dem hypothetischen Mehreinkommen der Frauen, wenn die Geschlechter bezüglich ihres Humankapitals gleichgestellt wären. Die verbleibende Einkommensdifferenz $\left(\hat{\boldsymbol{\beta}}_{M}-\hat{\boldsymbol{\beta}}_{F}\right)^{\prime} \overline{\boldsymbol{x}}_{F}$ bzw. $\left(\hat{\boldsymbol{\beta}}_{M}-\hat{\boldsymbol{\beta}}_{F}\right)^{\prime} \overline{\boldsymbol{x}}_{M}$ ist auf die unterschiedlichen geschlechtsspezifischen Lohnstrukturen zurückzuführen und entspricht dem unerklärten Teil des Lohnunterschiedes. Sie kann als Maß für die Diskriminierung interpretiert werden.

Ein Kritikpunkt am Ansatz nach OB besteht darin, dass entweder ausschließlich die Lohnstruktur der Männer oder die der Frauen als die nicht diskriminierte zugrunde gelegt wird, was zu unterschiedlichen Ergebnissen bei der Messung von Lohndiskriminierung führt. Da Diskriminierung sowohl Bevorzugung der Männer als auch Benachteiligung der Frauen beinhalten kann, wird alternativ vorgeschlagen, beide Lohnstrukturen zu verwenden und den Oaxaca-Blinder-Ansatz um einen Parametervektor $\hat{\boldsymbol{\beta}}^{*}$ zu erweitern:

$$
\overline{\ln y_{M}}-\overline{\ln y_{F}}=\underbrace{\hat{\boldsymbol{\beta}}^{{ }^{\prime}}\left(\overline{\boldsymbol{x}}_{M}-\overline{\boldsymbol{x}}_{F}\right)}_{\text {Ausstattungseffekt }}+\underbrace{\left(\hat{\boldsymbol{\beta}}_{M}-\hat{\boldsymbol{\beta}}^{*}\right)^{\prime} \overline{\boldsymbol{x}}_{M}+\left(\hat{\boldsymbol{\beta}}^{*}-\hat{\boldsymbol{\beta}}_{F}\right)^{\prime} \overline{\boldsymbol{x}}_{F}}_{\text {Diskriminierungseffekt }},
$$

wobei $\hat{\boldsymbol{\beta}}^{*}$ als gewichteter Durchschnitt der geschätzten Parametervektoren $\hat{\boldsymbol{\beta}}_{F}, \hat{\boldsymbol{\beta}}_{M}$ definiert ist und die Lohnstruktur ohne Diskriminierung darstellt:

$$
\hat{\boldsymbol{\beta}}^{*}=\boldsymbol{\Omega} \hat{\boldsymbol{\beta}}_{M}+(\mathbf{I}-\boldsymbol{\Omega}) \hat{\boldsymbol{\beta}}_{F}
$$

Wird beim Ansatz nach OB die Lohnstruktur der Männer als die nicht diskriminierte zugrunde gelegt, so gilt $\boldsymbol{\Omega}_{O B}=\mathbf{I}$, im anderen Fall $\boldsymbol{\Omega}_{O B}=\mathbf{0}$. Reimers (1983) gibt zu bedenken, dass die nicht diskriminierte Lohnstruktur zwischen der männlichen und weiblichen Lohnstruktur liegen sollte und wählt eine Gewichtungsmatrix $\Omega_{R}=0.5$ I. Cotton (1988) entscheidet sich für $\boldsymbol{\Omega}_{C}=s \mathbf{I}$, wobei $s$ dem Anteil der Männer in der Stichprobe darstellt, mit der Begründung, dass die nicht diskriminierte Lohstruktur mehr an die größere Gruppe angelehnt sein soll. Neumark (1988), Oaxaca und Ransom (1994) kommen zu dem Ergebnis, zur Schätzung des Parametervektors $\boldsymbol{\beta}^{*}$ aus Gleichung (3.4) die gepoolte Stichprobe von Männern und Frauen heranzuziehen, so dass

$$
\hat{\boldsymbol{\beta}}^{*}=\left(\boldsymbol{X}^{\prime} \boldsymbol{X}\right)^{-1} \boldsymbol{X}^{\prime} \boldsymbol{y}
$$




\begin{tabular}{lcc}
\hline \hline Methode & Stichprobe & Gewichtungsmatrix \\
\hline Oaxaca (1973), Blinder (1973) & männlich & $\boldsymbol{\Omega}_{O B}=\mathbf{I}$ \\
& weiblich & $\boldsymbol{\Omega}_{O B}=\mathbf{0}$ \\
Reimers (1983) & männlich/weiblich & $\boldsymbol{\Omega}_{R}=0.5 \mathbf{I}$ \\
Cotton (1988) & männlich/weiblich & $\boldsymbol{\Omega}_{C}=s \mathbf{I}$ \\
Neumark (1988), & männlich/weiblich & $\boldsymbol{\Omega}_{N}=\left(\boldsymbol{X}^{\prime} \boldsymbol{X}\right)^{-1}\left(\boldsymbol{X}_{M}^{\prime} \boldsymbol{X}_{M}\right)$ \\
Oaxaca und Ransom (1988, 1994) & & \\
\hline \hline
\end{tabular}

Tabelle 3.1: Zusammenfassung der Gewichtungsmatritzen

wobei $\boldsymbol{X}$ die Beobachtungsmatrix der Humankapitalcharakteristika und $\boldsymbol{y}$ den Vektor der logarithmierten Löhne der gepoolten Stichprobe darstellen. Es gilt:

$$
\begin{aligned}
\boldsymbol{\Omega}_{N} & =\left(\boldsymbol{X}^{\prime} \boldsymbol{X}\right)^{-1}\left(\boldsymbol{X}_{M}^{\prime} \boldsymbol{X}_{M}\right) \\
& =\left(\boldsymbol{X}_{M}^{\prime} \boldsymbol{X}_{M}+\boldsymbol{X}_{F}^{\prime} \boldsymbol{X}_{F}\right)^{-1}\left(\boldsymbol{X}_{M}^{\prime} \boldsymbol{X}_{M}\right) .
\end{aligned}
$$

Dabei entspricht $\boldsymbol{X}_{M}$ der Beobachtungsmatrix der Humankapitalcharakteristika der Gruppe der Männer und $\boldsymbol{X}_{F}$ der Beobachtungsmatrix der Gruppe der Frauen. Die Gewichtungsmatritzen nach Reimers und Cramer stellen genau dann Spezialfälle dar, wenn $0.5\left(\boldsymbol{X}^{\prime} \boldsymbol{X}\right)=\left(\boldsymbol{X}_{M}^{\prime} \boldsymbol{X}_{M}\right)$ bzw. $s\left(\boldsymbol{X}^{\prime} \boldsymbol{X}\right)=\left(\boldsymbol{X}_{M}^{\prime} \boldsymbol{X}_{M}\right)$.

\subsubsection{Empirische Resultate}

Die endogene Variable in den Lohnregressionen ist der logarithmierte Bruttostundenlohn. Dieser wird hier in Anlehnung an die Mincer-Lohngleichung durch die Anzahl der Ausbildungsjahre $(E d u)$, der Dauer der Betriebszugehörigkeit in Jahren als Polynom zweiten Grades (Ten, Ten_2) und der Berufserfahrung in Jahren ebenfalls als Polynom zweiten Grades (Exp, Exp_2) erklärt. Zusätzlich kommen als den Lohn erklärende Einflüsse die Variablen Erljob, Le200, Le2000, G2000, Ger und West in Frage. Bei der Auswahl der Lohnregressionsmodelle wird das Informationskriterium nach Akaike (AIC) herangezogen. Die in den Tabellen (3.2) und (3.3) aufgeführten Modelle weisen den niedrigsten AIC auf und werden für die weitere Studie verwendet. Die Stichprobe umfasst bei Männern 2931 Beobachtungen, die der Frauen 2840 Beobachtungen. Der Anteil der Männer und Frauen entspricht damit in etwa dem Anteil männlicher und weiblicher Arbeitnehmer unter den sozialversicherungspflichtigen Erwerbstätigen auf dem Arbeitsmarkt. Es wurden 416 
Männer und 414 Frauen aufgrund fehlender oder nicht plausibler Antworten ausgeschlossen.

\begin{tabular}{lrrrrl}
\hline \hline & Estimate & Std. Error & $\mathrm{t}$ value & $\operatorname{Pr}(>|\mathrm{t}|)$ & \\
\hline (Intercept) & 0.975 & 0.046 & 21.371 & 0.000 & $* * *$ \\
Edu & 0.062 & 0.003 & 21.111 & 0.000 & $* * *$ \\
Ten & 0.023 & 0.002 & 9.418 & 0.000 & $* * *$ \\
Ten_2 & -0.000 & 0.000 & -6.690 & 0.000 & $* * *$ \\
Exp & 0.023 & 0.003 & 8.135 & 0.000 & $* * *$ \\
Exp_2 & -0.000 & 0.000 & -5.826 & 0.000 & $* * *$ \\
Erljob & 0.081 & 0.015 & 5.358 & 0.000 & $* * *$ \\
Le200 & 0.144 & 0.021 & 7.001 & 0.000 & $* * *$ \\
Le2000 & 0.283 & 0.022 & 12.808 & 0.000 & $* * *$ \\
G2000 & 0.293 & 0.022 & 13.360 & 0.000 & $* * *$ \\
West & 0.339 & 0.018 & 18.827 & 0.000 & $* * *$ \\
\hline \hline
\end{tabular}

Tabelle 3.2: Koeffizientenschätzung für die Stichprobe der Männer (2006)

\begin{tabular}{lrrrrr}
\hline \hline & Estimate & Std.Error & t-value & $\operatorname{Pr}(>|\mathrm{t}|)$ & \\
\hline (Intercept) & 0.855 & 0.049 & 17.386 & 0.000 & $* * *$ \\
Edu & 0.063 & 0.003 & 20.912 & 0.000 & $* * *$ \\
Ten & 0.016 & 0.003 & 6.020 & 0.000 & $* * *$ \\
Ten_2 & -0.000 & 0.000 & -3.256 & 0.001 & $* *$ \\
Exp & 0.026 & 0.003 & 8.870 & 0.000 & $* * *$ \\
Exp_2 & -0.000 & 0.000 & -7.102 & 0.000 & $* * *$ \\
Erljob & 0.147 & 0.016 & 9.441 & 0.000 & $* * *$ \\
Le200 & 0.132 & 0.019 & 6.952 & 0.000 & $* * *$ \\
Le2000 & 0.246 & 0.021 & 11.641 & 0.000 & $* * *$ \\
G2000 & 0.294 & 0.021 & 13.881 & 0.000 & $* * *$ \\
West & 0.289 & 0.017 & 17.169 & 0.000 & $* * *$ \\
\hline \hline
\end{tabular}

Tabelle 3.3: Koeffizientenschätzung für die Stichprobe der Frauen (2006)

Da der Breusch-Pagan-Test einen klaren Hinweis auf Heteroskedastizität liefert, wird ein von MacKinnon und White (1985) vorgeschlagenes Verfahren zur Durchführung von Signifikanztests angewandt. Unter Verwendung des gewöhnlichen OLS-Schätzers kann die Kovarianzmatrix konsistent geschätzt werden, so dass zumindest asymtotisch korrekte Standardfehler und Tests resultieren. Sowohl bei Männern als auch bei Frauen haben die gewählten Einflussvariablen einen signifikanten Einfluss auf das Einkommen. Die Parameterschätzer der verwendeten Regressionsmodelle sind ebenfalls in den Tabellen (3.2) und (3.3) ersichtlich und folgendermaßen interpretierbar. Für jedes zusätzliche Bildungsjahr erhöht sich das Einkommen bei Männern und Frauen um 6.2\% bzw. 6.3 \%. Mit dem ersten Jahr Berufserfahrung nimmt der Lohn um 2.3\% bzw. 2.6\% zu, mit dem ersten Jahr Betriebszugehörigkeit um 2.3\% bzw 1.6\%. Bei weiterhin wachsender Berufserfahrung und 
Betriebszugehörigkeit erhöht sich das Einkommen mit geringfügig kleiner werdenden Zuwachsraten. Eine Tätigkeit im erlernten Beruf erhöht den Lohn der Männer um 8.4 \%, den der Frauen um 15.8 \%. Neben diesen individuellen Fähigkeiten bestimmen auch regionale und betriebliche Gegebenheiten das Einkommen. Beispielsweise verdienen beide Geschlechter, die in Großbetrieben mit mehr als 2000 Mitarbeitern beschäftigt sind im Vergleich zu Kleinbetrieben mit weniger als 20 Mitarbeitern um etwa 34 \% mehr. Männer, die in den alten Bundesländern tätig sind, erhalten einen um $40.4 \%$ höheren Lohn als Arbeiter in den neuen Bundesländern. Bei den Frauen beträgt der Unterschied $33.5 \%$.

\begin{tabular}{lccc}
\hline \hline Lohnunterschied (logarithmiert) & gesamt & erklärt & unerklärt \\
\hline OB mit Lohnstruktur der Frauen & 0.185 & 0.024 & 0.161 \\
OB mit Lohnstruktur der Männer & 0.185 & 0.034 & 0.151 \\
Reimers (1988) & 0.185 & 0.029 & 0.156 \\
Cotton (1988) & 0.185 & 0.029 & 0.156 \\
Neumark (1988) & 0.185 & 0.033 & 0.152 \\
\hline \hline
\end{tabular}

Tabelle 3.4: Ergebnisse der Zerlegung nach OB, Neumark (1988) (a)

In Tabelle (3.4) sind die Ergebnisse der Dekomposition dargestellt. Bei der Methode nach OB mit Lohnstruktur der Männer als nicht diskriminierter Lohnstruktur entfallen 0.034 Euro auf den erklärten und 0.151 Euro auf den unerklärten Teil der Lohnlücke, der damit $82 \%$ des Lohnunterschiedes ausmacht. Beim Verfahren nach OB mit Lohnstruktur der Frauen als nicht diskriminierter Lohnstruktur erhöht sich das Ausmaß der Diskriminierung auf 0.161 Euro (87 \%). Die Ergebnisse der übrigen Dekompositionsansätze liegen dazwischen. Sämtliche Verfahren zeigen, dass der weitaus größere Teil des Lohnunterschiedes auf Diskriminierung zurückzuführen ist und nicht auf Unterschiede in den Humankapitalausstattungen.

In Tabelle (3.5) ist dargestellt, welchen Einfluss die Kovariablen auf erklärten und unerklärten Teil der Lohnlücke bei der Dekomposition nach OB (1973) mit Lohnstruktur der Männer und Frauen sowie Neumark (1988) haben. Es fällt auf, dass bei sämtlichen Verfahren der auf den Intercept entfallene Anteil der Diskriminierung mit 0.120 Euro hoch ausfällt. 


\begin{tabular}{lrrrrrr}
\hline \hline & \multicolumn{2}{c}{ OB (Männer) } & \multicolumn{2}{c}{ OB (Frauen) } & \multicolumn{2}{c}{ Neumark } \\
& erklärt & unerklärt & erklärt & unerklärt & erklärt & unerklärt \\
\hline (Intercept) & 0.000 & 0.120 & 0.000 & 0.120 & 0.000 & 0.120 \\
Edu & -0.018 & -0.014 & -0.018 & -0.014 & -0.018 & -0.014 \\
Ten & 0.029 & 0.094 & 0.042 & 0.081 & 0.036 & 0.087 \\
Ten_2 & -0.017 & -0.055 & -0.030 & -0.042 & -0.023 & -0.049 \\
Eexp & 0.059 & -0.067 & 0.052 & -0.059 & 0.053 & -0.060 \\
Exp_2 & -0.052 & 0.071 & -0.039 & 0.058 & -0.041 & 0.059 \\
Erljob & -0.005 & -0.040 & -0.003 & -0.042 & -0.004 & -0.041 \\
Lt200 & -0.002 & 0.004 & -0.002 & 0.004 & -0.002 & 0.004 \\
Lt2000 & 0.006 & 0.009 & 0.006 & 0.008 & 0.006 & 0.008 \\
Ge2000 & 0.014 & -0.000 & 0.014 & -0.000 & 0.014 & -0.001 \\
West & 0.011 & 0.040 & 0.013 & 0.038 & 0.012 & 0.039 \\
\hline \hline
\end{tabular}

Tabelle 3.5: Ergebnisse der Zerlegung nach OB, Neumark (1988) (b)

\subsection{Berufliche Segregation berücksichtigende Verfah- ren zur Messung von Lohndiskriminierung}

Die in Abschnitt 3 vorgestellten Verfahren führen das Lohndifferential allein auf unterschiedliche Lohnstrukturen von Männern und Frauen zurück. Die nun vorgestellten Verfahren nach Brown, Moon und Zoloth (1980) sowie Appleton, Hoddinott und Krishnan (1999) liefern eine einfache Erweiterung des Ansatzes nach OB, indem sie die Lohnlücke auf die gesamte Verteilung der Berufe zerlegen und somit berücksichtigen, dass Frauen in größerem Maße schlechter bezahlte Berufe ausüben als es den Unterschieden in der Leistungsfähigkeit und Leistungswilligkeit entspricht.

\subsubsection{Dekomposition nach Brown, Moon und Zoloth (1980)}

\section{Methodologie}

Bei der Dekomposition nach Brown, Moon und Zoloth (1980) lässt sich die Lohnlücke als Differenz der gewichteten logarithmierten Löhne über sämtliche Berufe bzw. Berufsgruppen darstellen, wobei die Gewichtung dem Anteil der beschäftigten Frauen und Männer in den jeweiligen Berufen entspricht. Im Folgenden seien $P_{M j}$ und $P_{F j}$ der Anteil von Frauen oder Männern in einem Beruf $j=(1, \ldots K)$. Die Dekomposition lautet: 


$$
\begin{aligned}
\overline{\ln y_{M}}-\overline{\ln y_{F}} & =\sum_{j=1}^{K} P_{M j} \overline{\ln y_{M j}}-\sum_{j=1}^{K} P_{F j} \overline{\ln y_{F j}} \\
& =\sum_{j=1}^{K} P_{M j} \overline{\ln y_{M j}}+\sum_{j=1}^{K} P_{F j} \overline{\ln y_{M j}} \\
& -\sum_{j=1}^{K} P_{F j} \overline{\ln y_{M j}}-\sum_{j=1}^{K} P_{F j} \overline{\ln y_{F j}} \\
& =\underbrace{\sum_{j=1}^{K}\left(P_{M j}-P_{F j}\right) \overline{\ln y_{M j}}}_{\text {Verteilungseffekt }}+\underbrace{\sum_{j=1}^{K} P_{F j}\left(\overline{\ln y_{M j}}-\overline{\ln y_{F j}}\right)}_{\text {Lohneffekt }}
\end{aligned}
$$

Der Verteilungseffekt $\sum_{j=1}^{K}\left(P_{M_{j}}-P_{F j}\right){\overline{\ln y_{M}}}_{j}$ misst den Teil der Einkommenslücke, der auf eine unterschiedliche Verteilung auf die Berufe zurückzuführen ist. Er nimmt den Wert null an, wenn sich Männer und Frauen im selben Verhältnis auf die einzelnen Berufe aufteilen. Der Lohneffekt $\sum_{j=1}^{K} P_{F j}\left({\overline{\ln y_{M}}}_{j}-{\overline{\ln y_{F}}}_{j}\right)$ misst den Teil des Einkommensunterschiedes, der auf unterschiedliche mittlere logarithmierte Löhne in den unterschiedlichen Berufen zurückzuführen ist. Erhalten Männer und Frauen in jedem Beruf im Durchschnitt dasselbe Entgelt, so nimmt dieser Term ebenfalls den Wert null an.

Beide Effekte können sowohl in einen erklärten Teil und einen unerklärten Teil zerlegt werden. Es wird unterstellt, dass Männer ihre Berufe frei auswählen können und insofern die Verteilung männlicher Arbeiter auf die Berufe als die nicht diskriminierte angesehen werden kann. Im Folgenden sei $P_{M i j}$ die Wahrscheinlichkeit, mit der ein männlicher Arbeiter $i$ mit den Charakteristika $\boldsymbol{b}_{M}$ 円 in Beruf $j$ tätig ist. Die Schätzung der unbekannten Parametervektoren $\gamma_{M j}^{\prime}$ erfolgt mithilfe eines multinomialen Logitmodells:

$$
P_{M i j}=\frac{\exp \left(\boldsymbol{\gamma}_{M j}^{\prime} \boldsymbol{b}_{M i}\right)}{1+\sum_{s=1}^{K-1} \exp \left(\boldsymbol{\gamma}_{M s}^{\prime} \boldsymbol{b}_{M i}\right)}
$$

für $j=1, \ldots, K-1$ und

$$
P_{M i j}=\frac{1}{1+\sum_{s=1}^{K-1} \exp \left(\gamma_{M s}^{\prime} \boldsymbol{b}_{M i}\right)}
$$

für $j=K$ (Referenzkategorie).

\footnotetext{
${ }^{1}$ Die Variablen, die einen Einfluss auf die Berufswahl haben, sind in der Regel nicht identisch mit denjenigen, die die Lohnstruktur beeinflussen (siehe Empirische Resultate). Deshalb wird zwischen $\boldsymbol{b}$ und $\boldsymbol{x}$ unterschieden.
} 
Zur Ermittlung der Wahrscheinlichkeit $\hat{P}_{F i j}$, mit der eine weibliche Arbeitskraft mit den Charakteristika $\boldsymbol{b}_{F i}$ in Abwesenheit von Diskriminierung in Beruf $j$ tätig ist, werden die geschätzten Koeffizientenvektoren $\hat{\gamma}_{M j}$ herangezogen:

$$
\hat{P}_{F i j}=\frac{\exp \left(\hat{\boldsymbol{\gamma}}_{M j}^{\prime} \boldsymbol{b}_{F i}\right)}{1+\sum_{s=1}^{K-1} \exp \left(\hat{\boldsymbol{\gamma}}_{M s}^{\prime} \boldsymbol{b}_{F i}\right)}
$$

für $j=1, \ldots, K-1$ und

$$
\hat{P}_{F i j}=\frac{1}{1+\sum_{s=1}^{K-1} \exp \left(\hat{\gamma}_{M s}^{\prime} \boldsymbol{b}_{F i}\right)}
$$

für $j=K$

Im Folgenden entspricht $\hat{\bar{P}}_{F j}$ dem Mittelwert von $\hat{P}_{F i j}$ und stellt somit die geschätzte Verteilung von Frauen auf die Berufe in Abwesenheit von Diskriminierung dar. Brown, Moon und Zoloth (1980) zeigen, dass sich der Ausdruck $\overline{\ln y_{M}}-\overline{\ln y_{F}}$ folgendermaßen zerlegen lässt:

$$
\begin{aligned}
& \overline{\ln y_{M}}-\overline{\ln y_{F}}= \\
& \underbrace{\underbrace{K}_{\text {L=1 }}\left(P_{M j}-\hat{\bar{P}}_{F j}\right) \hat{\boldsymbol{\beta}}_{M j}^{\prime} \overline{\boldsymbol{x}}_{M j}+\underbrace{\sum_{j=1}^{K} P_{F j} \hat{\boldsymbol{\beta}}_{M j}^{\prime}\left(\overline{\boldsymbol{x}}_{M j}-\overline{\boldsymbol{x}}_{F j}\right)}_{\text {Lohneffekt }}}_{\text {Verteilungseffekt }} \\
& \text { Ausstattungseffekt } \\
& +\underbrace{\underbrace{\sum_{j=1}^{K} P_{F j}\left(\hat{\boldsymbol{\beta}}_{M j}-\hat{\boldsymbol{\beta}}_{F j}\right)^{\prime} \overline{\boldsymbol{x}}_{F j}}_{\text {Diskriminierungseffekt }}+\underbrace{\sum_{j=1}^{K}\left(\hat{\bar{P}}_{F j}-P_{F j}\right) \hat{\boldsymbol{\beta}}_{M j}^{\prime} \overline{\boldsymbol{x}}_{M j}}_{\text {Verteilungseffekt }}}_{\text {Lohneffekt }}
\end{aligned}
$$

\subsubsection{Dekomposition nach Appleton, Hoddinott und Krishnan (1999)}

\section{Methodologie}

Appleton, Hoddinott und Krishnan (1999) liefern eine Erweiterung des Ansatzes nach Brown, Moon und Zoloth (1980), indem das Lohndifferential folgendermaßen zerlegt wird:

$$
\begin{aligned}
\overline{\ln y_{M}}-\overline{\ln y_{F}}= & \sum_{j=1}^{K} P_{j}^{*}\left(\overline{\ln y_{M j}}-\overline{\ln y_{F j}}\right) \\
& +\sum_{j=1}^{K}\left(P_{M j}-P_{j}^{*}\right) \overline{\ln y_{M j}}+\sum_{j=1}^{K}\left(P_{j}^{*}-P_{F j}\right) \overline{\ln y_{F j}},
\end{aligned}
$$


wobei $P_{M j}, P_{F j}$ dem beobachteten Anteil von Männern und Frauen in den Berufen und $P_{j}^{*}$ der Verteilung auf die Berufe in Abwesenheit von Diskriminierung entspricht. Um $P_{j}^{*}$ zu schätzen werden für Männer und Frauen getrennt multinomiale Logitmodelle herangezogen. Im Folgenden seien $P_{M i j}, P_{F i j}$ die Wahrscheinlichkeiten mit der ein männlicher oder weiblicher Arbeiter $i$ mit den Humankapitalcharakteristika $\boldsymbol{b}_{M i}$ bzw. $\boldsymbol{b}_{F i}$ in Beruf $j$ tätig ist und $\gamma_{M}, \gamma_{F}$ die unbekannten, zu schätzenden Parametervektoren:

$$
P_{G i j}=\frac{\exp \left(\boldsymbol{\gamma}_{G j}^{\prime} \boldsymbol{b}_{G i}\right)}{1+\sum_{s=1}^{K-1} \exp \left(\gamma_{G s}^{\prime} \boldsymbol{b}_{G i}\right)}
$$

für $j=1, \ldots, K-1, G=M, W$ und

$$
P_{G i j}=\frac{1}{1+\sum_{s=1}^{K-1} \exp \left(\boldsymbol{\gamma}_{G s}^{\prime} \boldsymbol{b}_{G i}\right)}
$$

für $j=K$, (Referenzkategorie), $G=M, W$.

In Analogie zu Gleichung (3.7) der Dekomposition nach Neumark (1988) stellt $\boldsymbol{B}$ die Beobachtungsmatrix der Humankapitalcharakteristika der gepoolten Stichprobe und $\triangle$ eine Gewichtungsmatrix dar mit

$$
\triangle=\left(\boldsymbol{B}^{\prime} \boldsymbol{B}\right)^{-1} \boldsymbol{B}_{M}^{\prime} \boldsymbol{B}_{M}
$$

Entsprechend Gleichung (3.5) soll gelten:

$$
\hat{\boldsymbol{\gamma}}_{j}^{*}=\triangle \hat{\boldsymbol{\gamma}}_{M j}+(\boldsymbol{I}-\triangle) \hat{\boldsymbol{\gamma}}_{F j}
$$

für $j=1, \ldots, K-1$

Zur Ermittlung der Wahrscheinlichkeit $\hat{P}_{i j}^{*}$, mit der ein Individuum $i$ in Abwesenheit von Diskriminierung in Beruf $j$ tätig ist, werden die geschätzten Koeffizientenvektoren $\hat{\gamma}_{j}^{*}$ herangezogen:

$$
\hat{P}_{i j}^{*}=\frac{\exp \left(\hat{\boldsymbol{\gamma}}_{j}^{* \prime} \boldsymbol{b}_{i}\right)}{1+\sum_{s=1}^{K-1} \exp \left(\hat{\boldsymbol{\gamma}}_{s}^{* \prime} \boldsymbol{b}_{i}\right)}
$$

für $j=1, \ldots, K-1$, und

$$
\hat{P}_{i j}^{*}=\frac{1}{1+\sum_{s=1}^{K-1} \exp \left(\hat{\boldsymbol{\gamma}}_{s}^{* \prime} \boldsymbol{b}_{i}\right)}
$$

für $j=K$, (Referenzkategorie).

Im Folgenden entspricht $\hat{\bar{P}}_{M j}^{*}$ dem Mittelwert von $\hat{P}_{i j}^{*}$ bei männlichen Arbeitern und stellt somit die Verteilung von Männern auf die Berufe in Abwesenheit von Diskriminierung 
dar, $\hat{\bar{P}}_{F j}^{*}$ dem Mittelwert von $\hat{P}_{i j}^{*}$ bei weiblichen Arbeitern und $\hat{\bar{P}}_{j}^{*}$ dem Mittelwert von $\hat{P}_{i j}^{*}$ bei sämtlichen Arbeitern. Gleichung (3.12) kann nun folgendermaßen erweitert werden, wobei $\boldsymbol{\beta}_{j}^{*}$ den nicht diskriminierten Lohnstrukturen in den Berufen entspricht:

$$
\begin{aligned}
\overline{\ln y_{M}}-\overline{\ln y_{F}}= & \sum_{j=1}^{K} \hat{\bar{P}}_{j}^{*} \boldsymbol{\beta}_{j}^{* \prime}\left(\overline{\boldsymbol{x}}_{M j}-\overline{\boldsymbol{x}}_{F j}\right)+\sum_{j=1}^{K} \hat{\bar{P}}_{j}^{*}\left(\hat{\boldsymbol{\beta}}_{M j}-\hat{\boldsymbol{\beta}}_{j}^{*}\right)^{\prime} \overline{\boldsymbol{x}}_{M j} \\
& +\sum_{j=1}^{K} \hat{\bar{P}}_{j}^{*}\left(\hat{\boldsymbol{\beta}}_{j}^{*}-\hat{\boldsymbol{\beta}}_{F j}\right)^{\prime} \overline{\boldsymbol{x}}_{F j}+\sum_{j=1}^{K} \overline{\ln y_{M j}}\left(\hat{\bar{P}}_{M j}^{*}-\hat{\bar{P}}_{j}^{*}\right) \\
& +\sum_{j=1}^{K} \overline{\ln y_{F j}}\left(\hat{\bar{P}}_{j}^{*}-\hat{\bar{P}}_{F j}^{*}\right)+\sum_{j=1}^{K} \overline{\ln y_{M j}}\left(P_{M j}-\hat{\bar{P}}_{M j}^{*}\right) \\
& +\sum_{j=1}^{K} \overline{\ln y_{F j}}\left(\hat{\bar{P}}_{F j}^{*}-P_{F j}\right)
\end{aligned}
$$

Der erste Term misst den erklärten Teil des Lohnunterschiedes, der auf unterschiedliche mittlere logarithmierte Löhne in den Berufen zurückzuführen ist, der zweite und dritte Term den unerklärten auf unterschiedliche logarithmierte Löhne zurückzuführenden Teil. Der vierte und fünfte Term entsprechen dem auf berufliche Segregation zurückzuführenden erklärten Teil des Lohndifferentials, der sechste und siebte dem auf berufliche Segregation zurückzuführenden unerklärten Teil.

\subsubsection{Empirische Resultate}

Die in dieser Studie gewählten Berufsgruppen unterscheiden nach beruflichem Status, wobei das zugrunde liegende Skalenniveau stark mit der Treimann-Prestige-Skala ${ }^{2}$ korreliert. Es werden folgende Berufsgruppen gewählt:

- Gruppe 1: Tätigkeit mit geringem Status und wenig Eigenständigkeit.

- Gruppe 2: Beschäftigung mit einem geringen Maß an Spezialisierung.

- Gruppe 3: Tätigkeit, die eine gewisse Schulbildung erfordern und ein begrenztes Maß an Verantwortung einschließt.

- Gruppe 4: Leitende aber nicht prestigeträchtige Tätigkeit, die Fachhochschul oder Universitätsabschluss erfordert.

- Gruppe 5: Prestigeträchtige Tätigkeit mit umfassenden Führungsaufgaben.

\footnotetext{
${ }^{2}$ Der Prestigerang von Berufen wird in bildungssoziologischen Untersuchungen als relativ aussagekräftige Kategorie benutzt. Donald J. Treiman entwickelte den Standard Index of Occupational Prestige Scala (SIOPS) (auch Treiman-Index genannt) auf Grundlage des Klassifikationssystems ISCO von 1968.
} 
Bei der Auswahl der Lohnregressionsmodelle stehen dieselben Einflussvariablen wie bei der empirischen Analyse in Kapitel 3 zur Verfügung. Die Modelle, die in den jeweiligen Berufsgruppen bei Männern und Frauen den niedrigsten AIC aufweisen unterscheiden sich, allerdings ist jede Variable in mindestens einem der Modelle vertreten. Deshalb wird für die weitere Studie das volle Modell mit sämtlichen Einflussvariablen herangezogen. Auch wenn in den einzelnen Gruppen irrelevante Variablen berücksichtigt werden sind die Parameterschätzer unverzerrt. Allerdings wird bei der Schätzung an Genauigkeit verloren. Die für die Lohnregressionen verwendete Stichprobe entspricht der in Kapitel 3 verwendeten und umfasst bei Männern erneut 2931 Beobachtungen, die der Frauen 2840 Beobachtungen.

Aus den Parameterschätzungen geht hervor, dass bei sämtlichen Berufsgruppen die Löhne bei Männern und Frauen in Unternehmen mit mehr als 20 Mitarbeitern und einer Tätigkeit in den alten Bundesländern höher ausfallen. Bildungsjahre haben in Gruppe 1 mit dem niedrigsten Status keinen signifikanten Einfluss. In allen anderen Berufsgruppen steigt das Einkommen bei männlichen und weiblichen Arbeitnehmern mit höherer Bildung. Außerdem nimmt der Lohn bei Männern und Frauen mit wachsender Betriebszugehörigkeit und Berufserfahrung mit kleiner werdenden Zuwachsraten in sämtlichen Berufsgruppen zu.

Da bei den in dieser Studie gewählten Berufsgruppen die abhängige Variable Werte in geordneten Kategorien annimmt, wird bei der Dekomposition nach Brown, Moon und Zoloth (1980) die Ordinal-Regression angewandt, was sparsamere Parametrisierungen möglich macht. Bei der Auswahl der Einflussvariablen wird vermutet, dass Bildungsstand, Berufserfahrung und Betriebszugehörigkeit die Berufswahl beeinflussen, wobei eine Modellierung höheren Grades durchaus denkbar erscheint. Außerdem werden sämtliche in Kapitel 2 aufgeführten Dummyvariablen (Erljob, Le200, Le2000, G2000, Ger, West, Vakad, VaoA) herangezogen.

\begin{tabular}{lrrrrr}
\hline \hline & Estimate & Std. Error & $\mathrm{z}$ value & $\operatorname{Pr}(>|\mathrm{z}|)$ & \\
\hline Edu & 0.985 & 0.039 & 25.541 & 0.000 & $* * *$ \\
Edu_2 & -0.014 & 0.002 & -7.675 & 0.000 & $* * *$ \\
Ten & 0.028 & 0.005 & 5.684 & 0.000 & $* * *$ \\
Exp & 0.049 & 0.014 & 3.404 & 0.001 & $* * *$ \\
Exp_2 & -0.001 & 0.000 & -1.571 & 0.166 & \\
Erljob & 1.189 & 0.087 & 13.700 & 0.000 & $* * *$ \\
Lt200 & 0.231 & 0.114 & 2.031 & 0.042 & $*$ \\
Lt2000 & 0.128 & 0.121 & 1.057 & 0.291 & \\
Ge2000 & 0.451 & 0.121 & 3.720 & 0.000 & $* * *$ \\
West & 0.611 & 0.097 & 6.292 & 0.000 & $* * *$ \\
Ger & 0.632 & 0.184 & 3.433 & 0.001 & $* * *$ \\
VaoA & -0.533 & 0.124 & -4.304 & 0.000 & $* * *$ \\
\hline \hline
\end{tabular}

Tabelle 3.6: Koeffizientenschätzung für die Stichprobe der Männer 
Das in Tabelle (3.6) aufgeführte Modell weist den niedrigsten AIC auf und wird für die weitere Studie verwendet. Die Stichprobe der Männer umfasst 2530 Beobachtungen, die der Frauen 2476 Beobachtungen ${ }^{3}$ Die Parameterschätzer können folgendermaßen interpretiert werden. Die Wahrscheinlichkeit, einen Job mit höherem Status auszuüben, nimmt mit wachsender Bildung zu, mit steigender Berufserfahrung ebenfalls in den ersten 47 Jahren (in Tabelle (3.6) handelt es sich um gerundete Werte) und anschließend wieder ab. Die Jahre der Betriebszugehörigkeit, deutsche Staatszugehörigkeit, Tätigkeit im erlernten Beruf sowie in Unternehmen mit mehr als 20 Mitarbeitern erhöhen diese Wahrscheinlichkeit ebenfalls. Wenn der Vater ohne Berufsausbildung ist, verringert sich die Wahrscheinlichkeit, einen Job mit höherem Status auszuüben.

Bei der Dekomposition nach Appleton, Hoddinott und Krishnan (1999) erfolgt die Schätzung der Eintritsswahrscheinlichkeiten mit Hilfe des multinomialen Logit-Modells für kategoriale Kategorien, da eine Ordinalregression bei der Schätzung der Parametervektoren $\hat{\gamma}_{j}^{*}$ in Analogie zur Methode nach Neumark gemäß Gleichung (3.5) Schwierigkeiten bereitet. Bei der Berufswahl der Männer weist das Modell mit den schon bei der Dekomposition nach Brown, Moon und Zoloth (1980) verwendeten Variablen (vgl. Tabelle (3.6)) den niedrigsten AIC auf. Das bei der Berufswahl der Frauen vorzuziehende Modell unterscheidet sich dadurch, dass zusätzlich die Variable VaAkad in den linearen Prädiktor aufgenommen und die Variable Ger ausgeschlossen wird. Die Einflussvariablen der multinomialen Logitmodelle bei Männern und Frauen müssen jedoch übereinstimmen, damit die Parametervektoren $\hat{\gamma}_{j}^{*}$ ermittelt werden können. Deshalb wird für die weitere Studie das Modell mit sämtlichen Einflussvariablen, also zusätzlich mit den Variablen $\mathrm{VaAkad}$ und Ger verwendet.

\begin{tabular}{ccccccccc}
\hline \hline & \multicolumn{2}{c}{ beobachtet } & \multicolumn{2}{c}{ Brown et al. } & \multicolumn{2}{c}{ Appleton et al. } & \multicolumn{2}{c}{ log. Lohn } \\
Gruppe & Frauen & Männer & Frauen & Männer & Frauen & Männer & Frauen & Männer \\
\hline 1 & 0.127 & 0.161 & 0.128 & 0.161 & 0.111 & 0.134 & 2.177 & 2.452 \\
2 & 0.261 & 0.373 & 0.352 & 0.373 & 0.314 & 0.321 & 2.300 & 2.527 \\
3 & 0.520 & 0.262 & 0.295 & 0.262 & 0.436 & 0.395 & 2.627 & 2.788 \\
4 & 0.055 & 0.099 & 0.113 & 0.099 & 0.068 & 0.070 & 2.878 & 2.853 \\
5 & 0.038 & 0.105 & 0.111 & 0.105 & 0.070 & 0.079 & 3.109 & 3.350 \\
\hline \hline
\end{tabular}

Tabelle 3.7: Aufteilung von Frauen und Männern auf die Berufsgruppen

Tabelle (3.7) zeigt wie sich Frauen und Männer in den Stichproben sowie bei den beiden Dekompositionsansätzen in Abwesenheit von Diskriminierung auf die Berufe aufteilen. Zusätzlich sind die durchschnittlichen Bruttostundenlöhne ebenfalls nach Geschlechtern getrennt für sämtliche Berufsgruppen aufgeführt. Sie steigen mit zunehmenden Status an. Es fällt auf, dass in den Gruppen 1-3 sowie 5 das Einkommen der Männer weit über

\footnotetext{
${ }^{3}$ Da im Vergleich zu den Lohnregressionen andere Einflussvariablen verwendet werden, unterscheiden sich die aufgrund fehlender oder unplausibler Antworten ausgeschlossenen Arbeitnehmer. Dies hat zur Folge, dass die Stichproben verschieden sind.
} 
dem der Frauen liegt. In Berufsgruppe 4 verdienen weibliche Arbeitnehmer besser als männliche, wobei der Lohnunterschied geringer ausfällt als in den übrigen Gruppen. Der beobachtete Anteil der Frauen ist beim Dekompositionsansatz nach Brown, Moon und Zoloth (1980) in der Gruppe mit mittlerem Status höher als derjenige, der sich in Abwesenheit von Diskriminierung ergeben würde. In den Gruppen mit niedrigem oder hohem Status sind Frauen unterpräsentiert. Der beobachtete Anteil der Männer in den einzelnen Gruppen entspricht definitionsgemäß dem Anteil, der sich in Abwesenheit von Diskriminierung ergeben würde. Bei der Dekomposition nach Appleton, Hoddinott und Krishnan (1999) sind Frauen in der Gruppe mit mittlerem Status ebenfalls über- und in den übrigen Berufsgruppen unterpräsentiert. Der Unterschied fällt jedoch geringer aus als bei Brown, Moon und Zoloth (1980). Bei den Männern kehrt sich das Ergebnis um. Der beobachtete Anteil ist in der Gruppe mit mittlerem Status geringer als derjenige, der sich in Abwesenheit von Diskrinminierung ergeben würde. In den anderen Gruppen sind Männer überpräsentiert.

\begin{tabular}{lcccc}
\hline \hline & \multicolumn{2}{c}{ Ausstattungseffekt } & \multicolumn{2}{c}{ Diskriminierungseffekt } \\
& Verteilungseffekt & Lohneffekt & Verteilungseffekt & Lohneffekt \\
\hline Brown et al. & -0.021 & 0.043 & 0.020 & 0.143 \\
Appleton et al. & -0.006 & 0.048 & 0.001 & 0.140 \\
& & & & \\
\hline \hline
\end{tabular}

Tabelle 3.8: Ergebnisse der Dekomposition

Tabelle (3.8) zeigt die Ergebnisse der Dekomposition nach Brown, Moon und Zoloth (1980), Appleton, Hoddinott und Krishnan (1999). Die Ergebnisse der beiden Dekompositionsansätze sind vergleichbar. Wie in den Verfahren zuvor ist der größte Teil der Lohnlücke auf Diskriminierung und nicht auf unterschiedliche Humankapitalausstattungen zurückzuführen. Der Lohneffekt ist bedeutend ausgeprägter als der Verteilungseffekt. Frauen sind in Gruppen mit höherem Einkommen unterpräsentiert, was für Diskriminierung in Form eines ausgeprägten Verteilungseffektes sprechen würde. Allerdings wird dieses Resultat dadurch kompensiert, dass auch in den einkommensschwachen Berufsgruppen der Anteil der Frauen zu niedrig ist. Männer dagegen sind beim Verfahren nach Appleton, Hoddinott und Krishnan (1999) in diesen Gruppen überpräsentiert.

\subsection{Auf kontrafaktischen Dichteschätzungen basieren- de Methoden zur Messung von Lohndiskriminie- rung}

Die bisher vorgestellten, auf OLS-Schätzungen beruhenden Methoden zur Messung von Lohndiskriminierung, untersuchen, inwieweit unterschiedliche mittlere Löhne zwischen 
zwei Gruppen mit speziellen Merkmalsausprägungen auf Diskriminierung zurückzuführen sind. Hauptnachteil dieser Verfahren besteht darin, dass Einkommensunterschiede innerhalb der beiden Gruppen unberücksichtigt bleiben. Es ist aber durchaus von Interesse, inwieweit Lohndiskriminierung beispielsweise zwischen weiblichen und männlichen Spitzenoder Geringverdienern vorliegt. Die Verfahren nach DiNardo, Fortin und Lemieux (1996) sowie Machado und Mata (2005), Melly (2005) ermöglichen dagegen die Dekomposition des unkorrigierten Lohndifferentials nicht nur für die mittleren Löhne, sondern für sämtliche Quantile der Lohnverteilung. Eine solche Zerlegung bereitet insofern Schwierigkeiten, da zwar die Verteilungen der Löhne von Männern und Frauen beobachtbar sind, nicht aber die kontrafaktischen Lohnverteilungen, d.h. die Verteilung der Löhne von Männern mit Humankapitaleigenschaften von Männern und Lohnstruktur der Frauen bzw. die Verteilung der Löhne von Frauen mit Humankapitaleigenschaften von Frauen und Lohnstruktur der Männer.

\subsubsection{Dekomposition nach DiNardo, Fortin und Lemieux (1996)}

\section{Methodologie}

Beim Dekompositionsansatz nach DiNardo, Fortin und Lemieux (1996) werden die kontrafaktischen Lohnverteilungen mithilfe von gewichteten Kerndichteschätzungen ermittelt. Die Dichtefunktionen der logarithmierten Löhne von Männern und Frauen können ausgedrückt werden durch

$$
\begin{aligned}
f\left(\ln y_{M}\right) & =\int f\left(\ln y_{M} \mid \boldsymbol{x}\right) d F(\boldsymbol{x} \mid G=M) \\
f\left(\ln y_{F}\right) & =\int f\left(\ln y_{F} \mid \boldsymbol{x}\right) d F(\boldsymbol{x} \mid G=F),
\end{aligned}
$$

wobei $\boldsymbol{x}$ den $(1 \times k)$ Kovariablenvektor, $f\left(\ln y_{M} \mid \boldsymbol{x}\right), f\left(\ln y_{F} \mid \boldsymbol{x}\right)$ die bedingten Lohnverteilungen bei gegebenen Humankapitalausstattungen, $d F(\boldsymbol{x} \mid G=M), d F(\boldsymbol{x} \mid G=F)$ die Differentiale der Verteilungsfunktionen dieser Humankapitalcharakteristika bei gegebenem Geschlecht $G$ und $f\left(\ln y_{M}\right), f\left(\ln y_{F}\right)$ die entsprechenden Randverteilungen der Löhne für Männer und Frauen darstellen. Die kontrafaktische Lohnverteilung der Frauen entspricht

$$
f\left(\ln y_{F}^{c f}\right)=\int f\left(\ln y_{M} \mid \boldsymbol{x}\right) d F(\boldsymbol{x} \mid G=F),
$$

die kontrafaktische Lohnverteilung der Männer

$$
f\left(\ln y_{M}^{c f}\right)=\int f\left(\ln y_{F} \mid \boldsymbol{x}\right) d F(\boldsymbol{x} \mid G=M) .
$$

Wird bei der Dekomposition Gleichung (3.20) herangezogen, so definieren DiNardo, Fortin und Lemieux (1996) den Gewichtungsfaktor $\psi$ wie folgt: 


$$
\begin{aligned}
f\left(\ln y_{F}^{c f}\right) & =\int f\left(\ln y_{M} \mid \boldsymbol{x}\right) d F(\boldsymbol{x} \mid G=F) \\
& =\int \psi(\boldsymbol{x}) f\left(\ln y_{M} \mid \boldsymbol{x}\right) d F(\boldsymbol{x} \mid G=M)
\end{aligned}
$$

mit

$$
\psi(\boldsymbol{x}) \equiv \frac{d F(\boldsymbol{x} \mid G=F)}{d F(\boldsymbol{x} \mid G=M)}
$$

Unter Anwendung des Baysetheorems kann dieses Verhältnis ausgedrückt werden durch

$$
\psi(\boldsymbol{x})=\frac{P(G=F \mid \boldsymbol{x}) P(G=M)}{P(G=M \mid \boldsymbol{x}) P(G=F)} .
$$

Die bedingten Wahrscheinlichkeiten $P(G=M \mid \boldsymbol{x})$ und $P(G=F \mid \boldsymbol{x})$ stellen Propensity Scores für Männer und Frauen bei gegebenen Humankapitalcharakteristika dar und werden mittels eines geeigneten Probit- oder Logitmodells geschätzt. Die Wahrscheinlichkeiten $P(G=M)$ und $P(G=F)$ entsprechen dem Anteil von Männern und Frauen in der gepoolten Stichprobe. Die gewichtete Kerndichteschätzung der kontrafaktischen Lohnverteilung der Frauen lautet:

$$
\hat{f}\left(\ln y_{F}^{c f}\right)=\sum_{i \in M} \frac{1}{h} \psi(\boldsymbol{x}) K\left(\frac{y-\ln y_{i}}{h}\right),
$$

wobei die Stichprobe der Männer herangezogen wird.

Die Lohnlücke kann nun für ausgewählte Quantile der Lohnverteilungen $Q_{\theta}(\ln y)$ in einen erklärten und einen unerklärten Teil zerlegt werden

$$
\begin{aligned}
Q_{\theta}\left(\ln y_{M}\right)-Q_{\theta}\left(\ln y_{F}\right)= & {\left[Q_{\theta}\left(\ln y_{M}\right)-Q_{\theta}\left(\ln y_{F}^{c f}\right)\right]+} \\
& +\left[Q_{\theta}\left(\ln y_{F}^{c f}\right)-Q_{\theta}\left(\ln y_{F}\right)\right]
\end{aligned}
$$

Der Ausdruck $\left[Q_{\theta}\left(\ln y_{M}\right)-Q_{\theta}\left(\ln y_{F}^{c f}\right)\right]$ entspricht dem erklärten Teil des Lohnunterschiedes, $\left[Q_{\theta}\left(\ln y_{F}^{c f}\right)-Q_{\theta}\left(\ln y_{F}\right]\right.$ stellt den unerklärten Teil dar. Wird bei der Dekomposition die kontrafaktische Lohnverteilung der Männer herangezogen, so ist die Vorgehensweise analog. 


\section{Empirische Resultate}

Bei der Auswahl der Einflussvariablen des binären Logitmodells werden bei der Dekomposition nach DiNardo, Fortin und Lemieux (1996) als Einflussgrößen die schon in Kapitel 3 aufgeführten Variablen herangezogen, wobei das in Tabelle (3.9) aufgeführte Modell den niedrigsten AIC aufweist. Die Zielvariable Geschlecht nimmt den Wert 1 an, wenn die Merkmalsausprägung „weiblich“ ist. Die Stichproben entsprechen den schon in Kapitel 3 verwendeten und enthalten bei Männern 2931 und bei Frauen 2840 Beobachtungen.

\begin{tabular}{lrrrrr}
\hline \hline & Estimate & Std. Error & $\mathrm{z}$ value & $\operatorname{Pr}(>|\mathrm{z}|)$ & \\
\hline (Intercept) & -2.658 & 0.745 & -3.57 & 0.000 & $* * *$ \\
Edu & 0.432 & 0.110 & 3.93 & 0.000 & $* * *$ \\
Edu_2 & -0.014 & 0.004 & -3.61 & 0.000 & $* * *$ \\
Ten & 0.021 & 0.009 & 2.41 & 0.016 & $*$ \\
Ten_2 & -0.001 & 0.000 & -2.96 & 0.003 & $* *$ \\
Exp_2 & -0.000 & 0.000 & -5.43 & 0.000 & $* * *$ \\
Erljobj & 0.063 & 0.059 & 1.08 & 0.279 & \\
Lt200 & -0.139 & 0.075 & -1.86 & 0.063 & $*$ \\
Lt2000 & -0.280 & 0.082 & -3.43 & 0.001 & $* * *$ \\
Ge2000 & -0.386 & 0.082 & -4.73 & 0.000 & $* * *$ \\
West & -0.148 & 0.067 & -2.21 & 0.027 & $*$ \\
\hline \hline
\end{tabular}

Tabelle 3.9: Koeffizientenschätzung des Logitmodells der gepoolten Stichprobe

Die Wahrscheinlichleit weiblichen Geschlechts zu sein nimmt mit wachsenden Bildungsjahren und zunehmender Betriebszugehörigkeit innerhalb der ersten 15 Jahre zu und anschließend wieder ab. Eine Tätigkeit mit höchstens 20 Mitarbeitern, im erlernten Job und in den neuen Bundesländern erhöht diese Wahrscheinlichkeit ebenfalls.

\begin{tabular}{|c|c|c|c|c|c|c|}
\hline & $\overline{\theta \theta}$ & $10 \%$ & $25 \%$ & $50 \%$ & $75 \%$ & $90 \%$ \\
\hline & $\theta$-Quantil bei Männern & 2.089 & 2.406 & 2.716 & 3.006 & 3.258 \\
\hline & $\theta$-Quantil bei Frauen & 1.915 & 2.239 & 2.554 & 2.812 & 3.056 \\
\hline & Lohndifferential & 0.173 & 0.167 & 0.162 & 0.194 & 0.202 \\
\hline \multirow{3}{*}{$\begin{array}{l}\text { Dekomposition gemäß } \\
\text { Gleichung } 3.20\end{array}$} & $\theta$-Quantil kontrafaktisch & 2.037 & 2.357 & 2.675 & 2.975 & 3.230 \\
\hline & erklärter Teil & 0.051 & 0.049 & 0.041 & 0.031 & 0.028 \\
\hline & unerklärter Teil & 0.122 & 0.118 & 0.121 & 0.163 & 0.174 \\
\hline \multirow{3}{*}{$\begin{array}{l}\text { Dekomposition gemäß } \\
\text { Gleichung } 3.21\end{array}$} & $\theta$-Quantil kontrafaktisch & 1.948 & 2.268 & 2.573 & 2.828 & 3.071 \\
\hline & erklärter Teil & 0.033 & 0.029 & 0.019 & 0.016 & 0.014 \\
\hline & unerklärter Teil & 0.140 & 0.138 & 0.143 & 0.178 & 0.187 \\
\hline
\end{tabular}

Tabelle 3.10: Ergebnisse der Dekomposition nach DiNardo, Fortin und Lemieux (1996)

Die Kerndichteschätzungen basieren auf dem Gaußschen Kern, für die Wahl der optimalen 


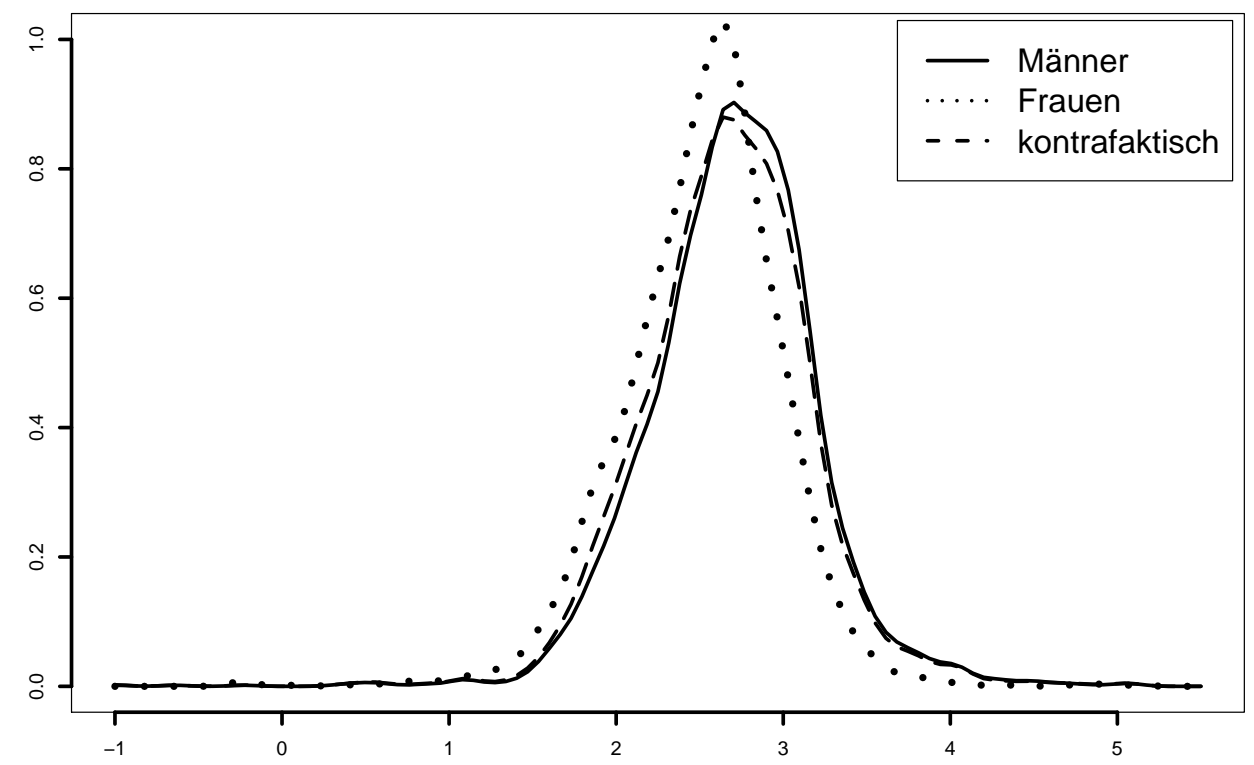

Abbildung 3.1: Dichtefunktionen der logarithmierten Bruttostundenlöhne

Bandbreite wurde die Regel von Silverman (Silverman's rule of thumb) verwendet. Abbildung (3.1) zeigt die (geschätzten) Verteilungen der logarithmierten Löhne von Männern und Frauen gemäß Gleichung (3.19) sowie die kontrafaktische Lohnverteilung mit Humankapital der Frauen und Lohnstruktur der Männer gemäß Gleichung (3.20) auf Basis des SOEP-Datensatzes für das Jahr 2006. Es fällt auf, dass die Verteilung der logarithmierten Löhne bei Frauen steiler als bei Männern verläuft und nach links verschoben ist, was zur Folge hat, dass die Quantile der Lohnverteilungen bei Männern größer sind als bei Frauen. Die kontrafaktische Verteilung liegt dazwischen.

In Tabelle (3.10) sind ausgewählte Quantile der drei zuvor mit Hilfe von Kerndichteschätzungen ermittelten Einkommensverteilungen dargestellt sowie die Ergebnisse der Dekomposition nach DiNardo, Fortin und Lemieux (1996). Die Quantile der kontrafaktischen Lohnverteilung mit Humankapital der Frauen und Lohnstruktur der Männer nach Gleichung (3.20) sind dichter an den Lohnquantilen der Männer als denen der Frauen. Wird bei der Dekomposition Gleichung (3.21) herangezogen, so kehrt sich das Bild um. Die Quantile der kontrafaktischen Lohnverteilung mit Humankapital der Männer und Lohnstruktur der Frauen sind nun dichter an den Lohnquantilen der Frauen als denen der Männer. Dies hat zur Folge, dass bei sämtlichen Quantilen der größte Teil der Lohnlücke auf Diskriminierung und nicht auf unterschiedliche Humankapitalausstat- 
tungen zurückzuführen ist. Das Lohndifferential und der unerklärte Teil dieser Einkommenslücke ist beim 90\% Quantil am Größten.

\subsubsection{Dekomposition nach Machado und Mata (2005), Melly (2005)}

\section{Methodologie}

Machado und Mata (2005) kombinieren bei ihrem Ansatz das Instrumentarium der von Koenker und Bassett (1978) eingeführten Quantilsregressionen mit einem BootstrapAnsatz. Stellt $\ln y_{i}$ den logarithmierten Lohn von Arbeiter $i$ dar und $\boldsymbol{x}_{i}$ den Vektor der zugehörigen Humankapitalcharakteristika, so können die bedingten Quantile wie folgt dargestellt werden:

$$
Q_{\theta}\left(\ln y_{i} \mid \boldsymbol{x}_{i}\right)=\boldsymbol{\beta}_{\theta}^{\prime} \boldsymbol{x}_{i}, \quad \theta \in(0,1) .
$$

Koenker und Basset (1978) zeigen, dass bei gegebenem $\theta$ der Parametervektor der Quantilsregression geschätzt werden kann durch

$$
\begin{aligned}
& \hat{\boldsymbol{\beta}}_{\theta}=\underset{\boldsymbol{\beta}_{\theta}}{\operatorname{argmin}}\left[\sum_{i: \ln } \theta\left|\ln y_{i} \geq \boldsymbol{\beta}_{\theta}^{\prime} \boldsymbol{x}_{i} \boldsymbol{\beta}_{\theta}^{\prime} \boldsymbol{x}_{i}\right|+\sum_{i: \ln y_{i}<\boldsymbol{\beta}_{\theta}^{\prime} \boldsymbol{x}_{i}}(1-\theta)\left|\ln y_{i}-\boldsymbol{\beta}_{\theta}^{\prime} \boldsymbol{x}_{i}\right|\right] \\
& =\underset{\boldsymbol{\beta}_{\theta}}{\operatorname{argmin}} \sum_{i} \rho_{\theta}\left(\boldsymbol{\beta}_{\theta}^{\prime} \boldsymbol{x}_{i}\right) .
\end{aligned}
$$

Die zweite zugrundeliegende Idee bei der Methode nach Machado und Mata (2005) ist das Theorem der Wahrscheinlichkeitsintegral-Transformation: Für $U$ uniform-verteilt in $(0,1)$ hat die Zufallsvariable $X=F^{-1}(U)$ die Verteilung F. Übertragen auf Lohndiskriminierungsmessungen bedeutet dies, dass bei gegebenem Kovariablenvektor $\boldsymbol{x}_{i}$ und zufälligem $\theta \sim U[0,1]$ die bedingte Lohnverteilung $\boldsymbol{\beta}_{\theta}^{\prime} \boldsymbol{x}_{i}$ aus Gleichung (3.27) die selbe Verteilung besitzt wie $\ln y_{i} \mid \boldsymbol{x}_{i}$. Machado und Mata (2005) zeigen, dass bei nicht fest vorgegebenem, sondern zufällig aus einer geeigneten Population gezogenem Kovariablenvektor $\boldsymbol{x}$ die Verteilung $\boldsymbol{\beta}_{\theta}^{\prime} \boldsymbol{x}$ der nicht bedingten Lohnverteilung $\ln y$ entspricht. Formal beinhaltet der Bootstrap-Ansatz nach Machado und Mata (2005) folgende Schritte:

1. Es wird eine Zufallsstichprobe vom Umfang $m$ aus $U(0,1): u_{1}, u_{2}, \ldots, u_{m}$ gezogen.

2. Für Männer und Frauen getrennt werden $m$ Koeffizientenvektoren: $\hat{\boldsymbol{\beta}}_{M u_{i}}$ und $\hat{\boldsymbol{\beta}}_{F u_{i}}$, $i=1, \ldots, m$ geschätzt in Übereinstimmung mit dem linearen Modell aus Gleichung (3.28).

3. Ebenfalls nach Geschlechtern getrennt werden $m$ Kovariablenvektoren mit Zurücklegen aus der Designmatrix $X$ der beobachteten Humankapitalcharakteristika gezogen und mit $\left\{\tilde{\boldsymbol{x}}_{M i}\right\}_{i=1}^{m},\left\{\tilde{\boldsymbol{x}}_{F i}\right\}_{i=1}^{m}$ bezeichnet. 
4. $\left\{\ln \tilde{y}_{M i}=\hat{\boldsymbol{\beta}}_{M u_{i}}^{\prime} \tilde{\boldsymbol{x}}_{M i}\right\}_{i=1}^{m}$ und $\left\{\ln \tilde{y}_{F i}=\hat{\boldsymbol{\beta}}_{F u_{i}}^{\prime} \tilde{\boldsymbol{x}}_{F i}\right\}_{i=1}^{m}$ stellen Zufallsstichproben vom Umfang $m$ aus den logarithmierten Lohnverteilungen $\ln y$ von Männern und Frauen dar. Bei $\left\{\ln \tilde{y}_{M i}=\hat{\boldsymbol{\beta}}_{M u_{i}}^{\prime} \tilde{\boldsymbol{x}}_{M i}\right\}_{i=1}^{m}$ wird sowohl zur Schätzung der Parametervektoren als auch bei der Ziehung der Kovariablenvektoren die Beobachtungsmatrix der Männer, bei $\left\{\ln \tilde{y}_{F i}=\hat{\boldsymbol{\beta}}_{F u_{i}}^{\prime} \tilde{\boldsymbol{x}}_{F i}\right\}_{i=1}^{m}$ die Beobachtungsmatrix der Frauen herangezogen.

5. $\left\{\ln \widetilde{y}_{F i}^{c f}=\hat{\boldsymbol{\beta}}_{M u_{i}}^{\prime} \tilde{\boldsymbol{x}}_{F i}\right\}_{i=1}^{m}$ und $\left\{\ln \widetilde{y}_{M i}^{c f}=\hat{\boldsymbol{\beta}}_{F u_{i}}^{\prime} \tilde{\boldsymbol{x}}_{M i}\right\}_{i=1}^{m}$ stellen eine Zufallsauswahl aus den kontrafaktischen Lohnverteilungen dar. Im Gegensatz zum vorherigen Schritt wird zur Schätzung der Parametervektoren die Beobachtungsmatrix der Männer und bei der Ziehung der Kovariablenvektoren die Beobachtungsmatrix der Frauen herangezogen oder umgekehrt.

Während Machado und Mata (2005) die Veränderungen der Lohnverteilung im Zeitverlauf analysieren, schlägt Melly (2005) eine Dekomposition der Lohnlücke vor, die einen Vergleich mit dem Ansatz nach OB vereinfacht. Die Schätzung der Lohnverteilungen erfolgt mithilfe von Kerndichteschätzungen. In Analogie zu Gleichung (3.26) der Dekomposition nach DiNardo, Fortin und Lemieux (1996) lässt sich die Lohnlücke wie folgt zerlegen:

$$
\begin{aligned}
Q_{\theta}\left(\ln y_{M}\right)-Q_{\theta}\left(\ln y_{F}\right)= & \underbrace{\left[Q_{\theta}\left(\ln \tilde{y}_{M}\right)-Q_{\theta}\left(\ln \tilde{y}_{F}^{c f}\right)\right]}_{\text {erklärter Teil }}+ \\
& +\underbrace{\left[Q_{\theta}\left(\ln \tilde{y}_{F}^{c f}\right)-Q_{\theta}\left(\ln \tilde{y}_{F}\right)\right]}_{\text {unerklärter Teil }}+r e s .
\end{aligned}
$$

bzw.

$$
\begin{aligned}
Q_{\theta}\left(\ln y_{M}\right)-Q_{\theta}\left(\ln y_{F}\right)= & \underbrace{\left[Q_{\theta}\left(\ln \tilde{y}_{M}\right)-Q_{\theta}\left(\ln \tilde{y}_{M}^{c f}\right)\right]}_{\text {unerklärter Teil }}+ \\
& +\underbrace{\left[Q_{\theta}\left(\ln \tilde{y}_{M}^{c f}\right)-Q_{\theta}\left(\ln \tilde{y}_{F}\right)\right]}_{\text {erklärter Teil }}+r e s .
\end{aligned}
$$

\section{Empirische Resultate}

Die Stichproben entsprechen erneut den schon bei der Dekomposition nach OB herangezogenen. Bei der Auswahl der Einflussvariablen für die Quantilsregressionen werden sämtliche in Kapitel 3 herangezogenen Einflussvariablen und zusätzlich die Variable ger verwendet. Bei den in dieser Studie betrachteten Lohnquantilen $\theta=(0,1,0.25,0.5,0.75,0.9)$ erhöht sich das Einkommen mit steigenden Bildungsjahren, zunehmender Betriebszugehörigkeit mit kleiner werdenden Zuwachsraten und Berufserfahrung ebenfalls mit kleiner 
werdenden Zuwachsraten, einer Tätigkeit im erlernten Beruf, in den alten Bundesländern und in Unternehmen mit mehr als 20 Mitarbeitern.

Abbildung (3.2) zeigt die (geschätzten) Verteilungen der logarithmierten Löhne von Männern und Frauen sowie die kontrafaktische Lohnverteilung mit Humankapital der Männer und Lohnstruktur der Frauen auf Basis des SOEP-Datensatzes für das Jahr 2006. Die zugrundeliegenden Stichproben sind nicht die tatsächlich beobachteten sondern diejenigen, die mithilfe von Bootstrapverfahren erzeugt wurden.

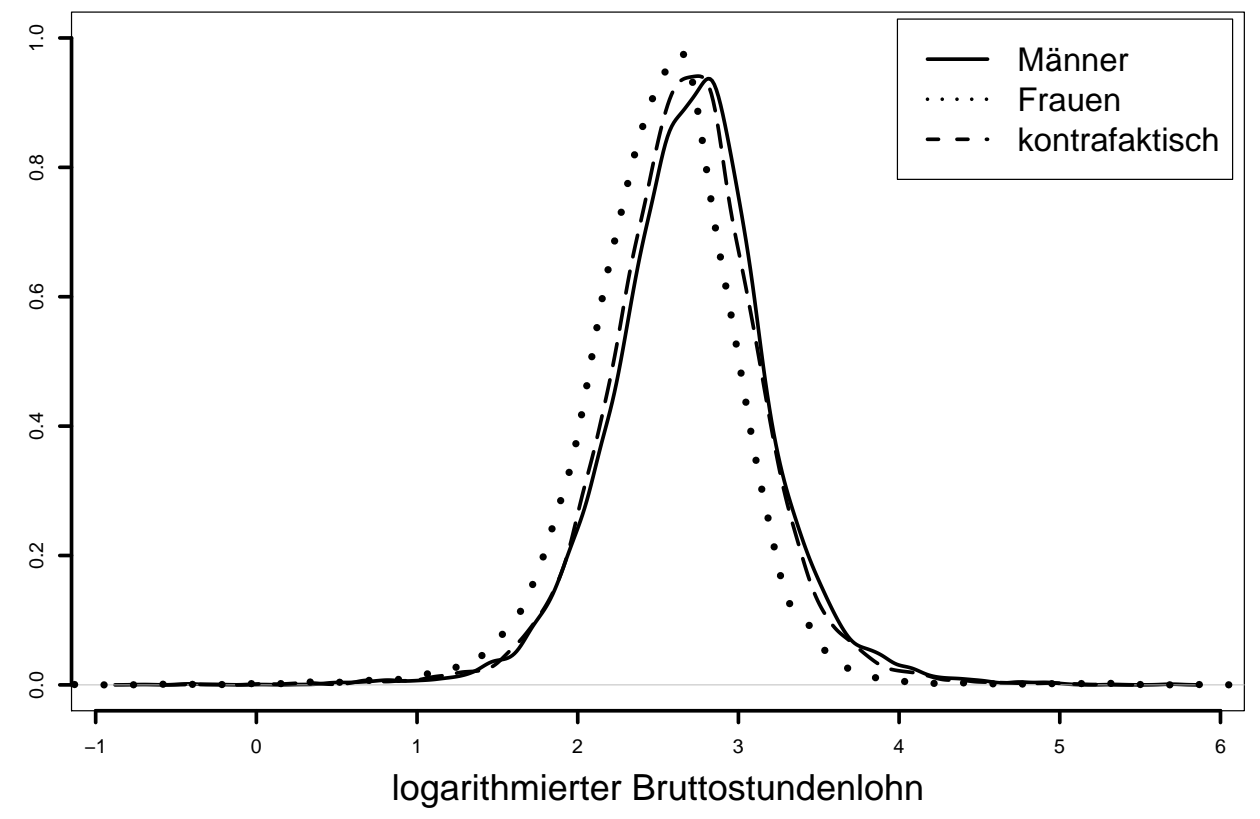

Abbildung 3.2: Dichtefunktionen der logarithmierten Bruttostundenlöhne

Die Kerndichteschätzungen basieren wie schon bei der Dekomposition nach DiNardo, Fortin und Lemieux (1996) auf dem Gaußschen Kern, für die Wahl der optimalen Bandbreite wurde erneut die Regel von Silverman (Silverman's rule of thumb) verwendet. Es fällt auf, dass sämtliche Verteilungen in etwa gleich steil verlaufen.

In Tabelle (3.11) sind ausgewählte Quantile der drei zuvor mit Hilfe von Kerndichteschätzungen ermittelten Einkommensverteilungen dargestellt sowie die Ergebnisse der Dekomposition nach Machado und Mata (2005), Melly (2005). Das Ergebnis der Dekomposition nach Machado und Mata (2005), Melly (2005) gleicht demjenigen nach DiNardo, Fortin und Lemieux (1996). Die Quantile der kontrafaktischen Lohnverteilung mit Humankapital der Frauen und Lohnstruktur der Männer sind dichter an den Lohnquantilen 
der Männer als denen der Frauen. Wird bei der Dekomposition die kontrafaktische Lohnverteilung mit Humankapital der Frauen und Lohnstruktur der Männer herangezogen, so kehrt sich das Bild um. Die Quantile dieser kontrafaktischen Lohnverteilung sind nun dichter an den Lohnquantilen der Frauen als denen der Männer. Dies hat wiederum zur Folge, dass bei sämtlichen Quantilen der größte Teil der Lohnlücke auf Diskriminierung und nicht auf unterschiedliche Humankapitalausstattungen zurückzuführen ist.

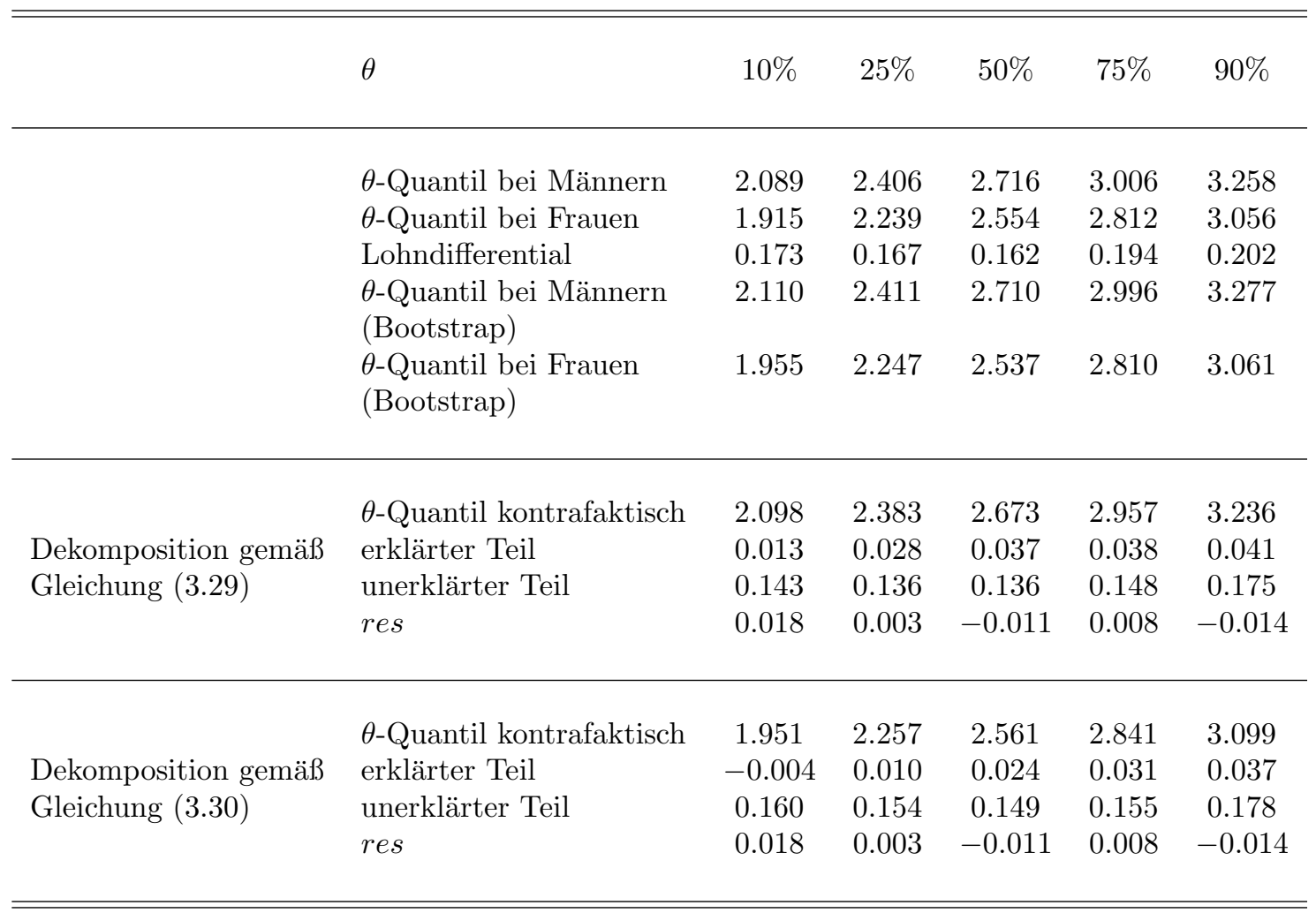

Tabelle 3.11: Ergebnisse der Dekomposition nach Machado und Mata (2005), Melly (2005)

\subsection{Auf Matching basierende Verfahren zur Messung von Diskriminierung}

\subsubsection{Methodologie}

Ein potentielles Problem des Ansatzes nach OB ist die Gültigkeit der Annahme über die funktionale Form der bedingten Erwartungswerte der Löhne bzw. logarithmierten Löhne. Werden die hypothetischen, in der Realität nicht beobachtbaren Löhne $\hat{\boldsymbol{\beta}}_{F}^{\prime} \overline{\boldsymbol{x}}_{M}$ bzw. $\hat{\boldsymbol{\beta}}_{M}^{\prime} \overline{\boldsymbol{x}}_{F}$ geschätzt, so wird angenommen, dass es möglich ist, Frauen zu finden, deren Humankapitalausstattungen vergleichbar sind mit denen der Männer und umgekehrt. Allerdings entsteht ein Problem der Vergleichbarkeit, da einige bei Männern übliche Kombinationen 
unterschiedlicher Charaktereigenschaften bei Frauen nicht beobachtbar sind. Bei Anwendung der Methode nach OB wird unterstellt, dass bei der Schätzung der hypothetischen Löhne die Lohnstrukturen $\hat{\boldsymbol{\beta}}_{M}^{\prime}$ bzw. $\hat{\boldsymbol{\beta}}_{F}^{\prime}$ auch dort gelten, wo keine Kombinationen der entsprechenden Humankapitalausstattungen anzutreffen sind. Der Dekompositionsansatz nach Nopo (2008) stellt eine nicht parametrische Alternative des Verfahrens nach OB dar und benötigt keine Schätzung der Lohnverteilungen. Es wird zwischen Individuen unterschiedlicher Gruppen, deren Humankapitalcharakteristika sich nicht unterscheiden ("common support") und Individuen außerhalb dieses Bereiches ("out-of-support") unterschieden. Sämtliche Beobachtungen werden in der weiteren Analyse mit einbezogen.

Im Folgenden sei $g_{M}(\boldsymbol{x})=E(\ln y \mid \boldsymbol{x}, M)$ der mittlere Lohn der Männer mit Humankapitalausstattungen $\boldsymbol{x}, F_{M}(\boldsymbol{x})$ die gemeinsame Verteilungsfunktion dieser Charakteristika und $S_{M}$ der Bereich der beobachteten Kombinationen von Humankapitalcharakteristika bei Männern. Wir definieren $g_{F}(\cdot), F_{F}(\cdot)$ und $S_{F}(\cdot)$ auf dieselbe Weise für Frauen. $S=S_{M} \cap S_{F}$ bezeichnet den "common support", $P_{S \mid M}=P(X \in S \mid M)=\int_{S} d F_{M}(\boldsymbol{x})$ die Wahrscheinlichkeit der Menge $S$ unter der Verteilung $d F_{M}(\cdot)$. Die Männer gehören nun entweder dem common support $S$ oder einem Bereich $\bar{S}$ außerhalb des common supports an. Es gilt

$$
E(\ln y \mid M)=E_{S}(\ln y \mid M) P_{S \mid M}+E_{\bar{S}}(\ln y \mid M) P_{\bar{S} \mid M} .
$$

Da $P_{S \mid M}=1-P_{\bar{S} \mid M}$ lässt sich Gleichung (3.31) wie folgt umformen:

$$
E(\ln y \mid M)=P_{\bar{S} \mid M}\left[E_{\bar{S}}(\ln y \mid M)-E_{S}(\ln y \mid M)\right]+E_{S}(\ln y \mid M) .
$$

Bei derselben Vorgehensweise erhalten wir für den mittleren Lohn der Frauen

$$
E(\ln y \mid F)=P_{\bar{S} \mid F}\left[E_{\bar{S}}(\ln y \mid F)-E_{S}(\ln y \mid F)\right]+E_{S}(\ln y \mid F)
$$

Es gilt:

$$
\begin{aligned}
\triangle= & E(\ln y \mid M)-E(\ln y \mid F) \\
= & \underbrace{\left[E_{S}(\ln y \mid M)-E_{S}(\ln y \mid F)\right]}_{I}+\underbrace{P_{\bar{S} \mid M}\left[E_{\bar{S}}(\ln y \mid M)-E_{S}(\ln y \mid M)\right]}_{I I} \\
& +\underbrace{P_{\bar{S} \mid F}\left[E_{S}(\ln y \mid F)-E_{\bar{S}}(\ln y \mid F)\right]}_{I I I}
\end{aligned}
$$

Term $I$ beschreibt Lohnunterschiede innerhalb des common supports, Term II und III Einkommensdifferenzen außerhalb des common supports. Der Lohnunterschied in Ausdruck $I$ lässt sich, vergleichbar mit der Methode nach OB, in einen erklärten und einen unerklärten Teil zerlegen: 


$$
\begin{aligned}
E_{S}(\ln y \mid M)-E_{S}(\ln y \mid F)= & \int_{S} g_{M}(\boldsymbol{x}) d F_{M}(\boldsymbol{x})-\int_{S} g_{F}(\boldsymbol{x}) d F_{F}(\boldsymbol{x}) \\
= & \int_{S} g_{M}(\boldsymbol{x})\left[d F_{M}(\boldsymbol{x})-d F_{F}(\boldsymbol{x})\right] \\
& +\int_{S}\left[g_{M}(\boldsymbol{x})-g_{F}(\boldsymbol{x})\right] d F_{F}(\boldsymbol{x}) .
\end{aligned}
$$

Beim Dekompositionsansatz nach Nopo (2008) umfasst der Lohnunterschied der mittleren Löhne zwischen Männern und Frauen vier Komponenten

$$
\triangle=\triangle_{M}+\triangle_{\boldsymbol{x}}+\triangle_{0}+\triangle_{F}
$$

mit

$$
\begin{aligned}
\triangle_{M} & =P_{\bar{S} \mid M}\left[E_{\bar{S}}(\ln y \mid M)-E_{S}(\ln y \mid M)\right], \\
\triangle_{X} & =\int_{S} g_{M}(\boldsymbol{x})\left[d F_{M}(\boldsymbol{x})-d F_{F}(\boldsymbol{x})\right], \\
\triangle_{0} & =\int_{S}\left[g_{M}(\boldsymbol{x})-g_{F}(\boldsymbol{x})\right] d F_{F}(\boldsymbol{x}), \\
\triangle_{F} & =P_{S \mid F}\left[E_{\bar{S}}(\ln y \mid F)-E_{\bar{S}}(\ln y \mid F)\right],
\end{aligned}
$$

wobei $\triangle_{M}, \triangle_{\boldsymbol{x}}, \triangle_{F}$ auf unterschiedliche beobachtete Humankapitalausstattungen und $\triangle_{0}$ auf unterschiedliche unbeobachtete Charakteristika oder auf Diskriminierung zurückzuführen sind.

Die Komponente $\triangle_{M}$ beschreibt den Teil der Lohnlücke, der sich durch Unterschiede zwischen Männern innerhalb des common supports und Männern außerhalb dieses Bereiches erklären lässt. $\triangle_{M}$ nimmt den Wert null an, wenn sämtliche Männer dem common support angehören oder aber wenn die mittleren Löhne von Männern innerhalb und außerhalb des common supports identisch sind. $\triangle_{M}$ wird ermittelt als Unterschied zwischen erwarteten logarithmierten Löhnen von Männern innerhalb und außerhalb des common supports, gewichtet mit der Wahrscheinlichkeit mit der Männer dem common support angehören.

Die Komponente $\triangle_{\boldsymbol{x}}$ beschreibt den durch unterschiedliche Humankapitalausstattungen erklärten Lohnunterschied zwischen Männern und Frauen innerhalb des common supports und entspricht $\hat{\boldsymbol{\beta}}_{M}^{\prime}\left(\overline{\boldsymbol{x}}_{M}-\overline{\boldsymbol{x}}_{F}\right)$ bei der Dekomposition nach OB. Der Term $\triangle_{0}$ beschreibt die unerklärte Einkommensdifferenz zwischen Männern und Frauen und entspricht $\left(\hat{\boldsymbol{\beta}}_{M}-\hat{\boldsymbol{\beta}}_{F}\right)^{\prime} \overline{\boldsymbol{x}}_{F}$ bei der Methode nach OB.

Die Komponente $\triangle_{F}$ ist auf unterschiedliche mittlere Löhne von Frauen innerhalb und außerhalb des common supports zurückzuführen. 
Die in Gleichung (3.12) vorgestellten, die Lohnlücke beschreibenden, vier Komponenten werden mit Hilfe eines von Nopo (2008) eingeführten Matching-Verfahrens ermittelt. Die Frauen werden ohne Zurücklegen aus dem ursprünglichen Datensatz geresamplet und jeder Beobachtung wird eine synthetische männliche Vergleichsperson angepasst, deren Charakteristika dem Durchschnitt sämtlicher ausgewählter Männer entspricht. Der von Nopo (2008) vorgeschlagene Matching-Algorithmus kann folgendermaßen zusammengefasst werden.

1. Es wird eine Frau aus der Stichprobe der Frauen ohne Zurücklegen ausgewählt.

2. Es werden sämtliche Männer mit vergleichbaren Humankapitalcharakteristika der zuvor gezogenen Frau ausgewählt.

3. Mit den in Schritt 2 ausgewählten Individuen wird eine künstliche männliche Person gebildet und mit der Original-Frau verglichen.

4. Die Beobachtungen der beiden Individuen (Original-Frau und künstlich geschaffene Vergleichsperson) gelangen in die entsprechende Stichprobe übereinstimmender Individuen.

5. Schritt 1-4 werden solange wiederholt bis sich keine Frau mehr in der Originalstichprobe der Frauen befindet.

Als Resultat der Anwendung des oben beschriebenen 1:k Matchings wird der ursprüngliche Datensatz aufgeteilt in Männer und Frauen, die miteinander verglichen werden können $((M$, matched $),(F$, matched $))$ sowie in Männer und Frauen, für die keine entspechenden gegengeschlechtlichen Vergleichspersonen existieren ( $M$, unmatched $),(F$, unmatched $))$. Die Aufteilung der Frauen innerhalb und außerhalb des common supports sowie die Bildung synthetischer männlicher Vergleichspersonen ermöglicht, die kontrafaktische Lohnverteilung der Frauen, d.h. die Verteilung der Löhne von Frauen mit Humankapitaleigenschaften von Frauen und Lohnstruktur der Männer zu schätzen. Auf diese Weise lassen sich die in Gleichung (3.12) vorgestellten vier Komponenten mithilfe bedingter Erwartungswerte und der empirischen Häufigkeiten $p_{M}$ (unmatched) sowie $p_{F}$ (unmatched) bestimmen ohne die Lohngleichungen $g_{M}(\boldsymbol{x})$ und $g_{F}(\boldsymbol{x})$ schätzen zu müssen:

$$
\begin{aligned}
\triangle_{M} & =p_{M}(\text { unmatched })\left[E_{M, \text { unmatched }}(\ln y \mid M)-E_{M, \text { matched }}(\ln y \mid M)\right] \\
\triangle_{x} & =E_{M, \text { matched }}(\ln y \mid M)-E_{F, \text { matched }}(\ln y \mid M) \\
\triangle_{0} & =E_{F, \text { matched }}(\ln y \mid M)-E_{F, \text { matched }}(\ln y \mid F) \\
\triangle_{F} & =p_{F}(\text { unmatched })\left[E_{F, \text { matched }}(\ln y \mid F)-E_{F, \text { unmatched }}(\ln y \mid F)\right]
\end{aligned}
$$

Der Ansatz nach Nopo (2008) benötigt keine parametrischen Annahmen und basiert ausschließlich auf der Modellannahme, dass Individuen mit denselben beobachteten Humankapitalcharakteristika dieselbe Bezahlung erhalten sollten ungeachtet des Geschlechts. 


\subsubsection{Empirische Resultate}

In dieser Studie wird beim Dekompositionsansatz nach Nopo (2008) zur Messung des Unterschiedes zwischen den Humankapitalcharakteristika von männlichen und weiblichen

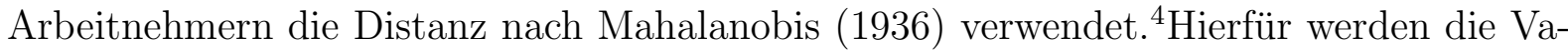
riablen Edu, Exp, Ten, Erljob, Le20, Ger und West herangezogen. Da es sich bei den zu vergleichenden Merkmalen um quantitative oder zumindest dichotome Variablen handelt, die wie quantitative Variablen behandelt werden können, ist die Mahalanobisdistanz als Distanzmaß zulässig. Die Stichproben entsprechen den schon zuvor verwendeten mit 2931 männlichen Arbeitnehmern und 2840 Arbeitnehmerinnen. Die Entscheidung, ob Objekte miteinander verglichen werden können, hängt von einem im Vorfeld festgelegten Toleranzbereich ab. Das Ergebnis der Dekomposition nach Nopo (2006) ist in Tabelle (3.12) bei unterschiedlich gewählten Toleranzbereichen wiedergegeben.

\begin{tabular}{cccccccc}
\hline \hline$\overline{\ln y_{M}}-\overline{\ln y_{F}}$ & $\begin{array}{c}\text { Schwellen- } \\
\text { wert }\end{array}$ & $\begin{array}{c}\text { Männer } \\
\text { matched }\end{array}$ & $\begin{array}{c}\text { Frauen } \\
\text { matched }\end{array}$ & $\triangle_{M}$ & $\triangle_{F}$ & $\triangle_{x}$ & $\triangle_{O}$ \\
\hline 0.185 & 0.00 & 0.003 & 0.004 & -0.091 & 0.347 & 0.042 & -0.113 \\
0.185 & 0.01 & 0.229 & 0.236 & -0.027 & 0.058 & 0.022 & 0.132 \\
0.185 & 0.05 & 0.573 & 0.570 & 0.004 & 0.026 & 0.010 & 0.145 \\
0.185 & 0.10 & 0.750 & 0.767 & 0.006 & 0.012 & 0.035 & 0.132 \\
0.185 & 5.00 & 0.997 & 0.999 & -0.001 & 0.000 & 0.037 & 0.149 \\
0.185 & 70.0 & 1 & 1 & NA & NA & 0 & 0.185 \\
\hline \hline
\end{tabular}

Tabelle 3.12: Ergebnisse der Dekomposition nach Nopo (2006)

In dieser Studie nimmt die Mahalanobisdistanz Werte zwischen 0 und 69 an. Wird der Schwellenwert gleich null gesetzt, d.h. es werden Männer und Frauen mit identischen Eigenschaften verglichen, ist aufgrund einer Vielzahl von Merkmalen der Anteil von Männern und Frauen, die dem common support zugehören, sehr gering und beträgt 0.3 bzw. $0.4 \%$. Für 10 männliche und 11 weibliche Arbeitnehmer/innen existieren gegengeschlechtliche statistische Zwillinge. Ein negativer Term $\triangle_{M}$ bedeutet, dass Männer außerhalb des common supports im Durchschnitt weniger verdienen als Männer innerhalb des common supports. Dies ist insofern bemerkenswert, da davon ausgegangen werden kann, dass Männer

\footnotetext{
${ }^{4}$ Die Mahalanobisdistanz ermittelt nicht nur den Abstand zwischen zwei Objekten in einem Merkmal, sondern berücksichtigt auch die Varianz jedes Merkmals und eventuelle Korrelationen mit anderen Merkmalen. Je größer die Gesamtvarianz eines Merkmals ist, desto geringer wird die entsprechende Differenz $\left(x_{n i}-x_{n j}\right)$ für die entsprechende Gesamtdistanz zwischen den Objekten $i$ und $j$ gewichtet. Sind zwei Merkmale hoch korreliert, d.h. sie liefern beide annähernd die selbe Information, wird der gemeinsame Erklärungsbeitrag an der Distanz entsprechend niedrig gewichtet. Ein weiterer Vorteil der Mahalanobisdistanz ist Skalierungsinvarianz. Die Distanz wird ermittelt aus der Summe der Differenzen der einzelnen Merkmale, gewichtet mit der inversen Varianz-Kovarianzmatrix:
}

$$
M D_{i j}=\left(\boldsymbol{x}_{i}-\boldsymbol{x}_{j}\right)^{\prime} \mathbf{C o v}^{-1}\left(\boldsymbol{x}_{i}-\boldsymbol{x}_{j}\right) .
$$

Dabei bezeichnen $\boldsymbol{x}_{i}$ und $\boldsymbol{x}_{j}$ die Merkmalsvektoren der Objekte $i$ und $j$ und Cov die Varianz-KovarianzMatrix. 
ohne weibliche Vergleichspersonen mehr Humankapital aufweisen und deshalb auch höhere Löhne erhalten würden als Männer mit weiblicher Vergleichsperson. Für Frauen erhalten wir das erwartete Ergebnis. Der Term $\triangle_{F}$ ist ebenfalls negativ, d.h., dass Arbeitnehmerinnen außerhalb des common supports im Durchschnitt weniger verdienen als Frauen innerhalb des common supports. Der Unterschied der mittleren Löhne zwischen Männern und Frauen innerhalb des common supports beträgt -0.071 Euro. Frauen verdienen dementspechend mehr als Männer. Das Ergebnis der Dekomposition nach Nopo (2006) kehrt bei einem gewählten Schwellenwert von null die Resultate der vorherigen Methoden um. Männer werden diskriminiert, wobei das Ausmaß der Diskriminierung mit 0.113 Euro sogar höher ausfällt als die Lohnlücke innerhalb des common supports. Dieses Ergebnis darf nicht überinterpretiert werden. Da insgesamt nur 11 Frauen dem common support zugehören, fällt eine Arbeitnehmerin mit sehr hohem Lohn stark ins Gewicht mit der Folge, dass sich die Einkommensdifferenz und der Anteil der Diskriminierung gegenüber Männern erhöht.

Wird der Schwellenwert gleich 70 gesetzt, werden jeder Frau sämtliche Männer als Vergleichspersonen zugeordnet mit der Folge, dass alle Arbeitskräfte dem common support zugehören. Die Terme $\triangle_{F}$ und $\triangle_{M}$ können nicht ermittlt werden. Da $\triangle_{x}$ gleich 0 und $\triangle_{O}$ dem Ausmaß der Lohnlücke entspricht, ist beim gewählten Schwellenwert die gesamte Einkommensdifferenz auf Diskriminierung zurückzuführen.

Bei den ansonsten gewählten Toleranzbereichen sind die Ergebnisse der Dekomposition nach Nopo (2008) vergleichbar mit den zuvor beschriebenen Verfahren. Der weitaus größte Teil der Lonlücke ist auf Diskriminierung und nicht auf unterschiedliche Humankapitalausstattungen zurückzuführen.

Das Dekompositionsverfahren nach Nopo ist dementsprechend sehr kritisch zu bewerten. Bei einer Vielzahl von Merkmalen kann es schwierig sein, statistische Zwillinge zu finden. Die Güte einer auf der Bildung von Vergleichsgruppen basierenden Schätzung wird jedoch stets davon abhängig sein, inwieweit es gelingt, mit den beobachteten Merkmalen Vergleichbarkeit zwischen weiblichen und männlichen Vergleichspersonen herzustellen. Das oben beschriebene Matching-Verfahren stößt demzufolge dann an seine Grenzen, wenn eine Überlappung der Merkmale zwischen den zu vergleichenden Gruppen nicht oder in einem nur sehr geringen Maß gegeben ist.

\subsection{Verfahren zur Analyse von Diskriminierung im Zeitverlauf}

\subsubsection{Methodologie}

Die Methode nach Juhn, Murphy und Pierce (1991) ermöglicht, Veränderungen der Lohnlücke im Zeitverlauf zu analysieren. Juhn, Murphy und Pierce (1991) führen die Lohnlücke auf 
Unterschiede in den beobachtbaren Humankapitalcharakteristika und auf unbeobachtete individuelle Fähigkeiten zurück, die sich in unterschiedlichen Residuen niederschlagen. Sie unterstellen für Männer und Frauen dieselben Ertragsraten $\boldsymbol{\beta}$, so dass zum Zeitpunkt $t \boldsymbol{\beta}_{M t}=\boldsymbol{\beta}_{F t}=\boldsymbol{\beta}_{t}$ gilt. Zur Schätzung des unbekannten Parametervektors $\boldsymbol{\beta}$ wird die Stichprobe der Männer herngezogen:

$$
\begin{aligned}
\ln y_{M i t} & =\boldsymbol{\beta}_{t}^{\prime} \boldsymbol{x}_{M i t}+u_{M i t}, \\
\ln y_{F i t} & =\boldsymbol{\beta}_{t}^{\prime} \boldsymbol{x}_{F i t}+u_{F i t} .
\end{aligned}
$$

Im Folgenden entsprechen $\hat{\theta}_{M i t}, \hat{\theta}_{F i t}$ den geschätzten standardisierten Residuen für Individuum $i$ und $\hat{\sigma}_{t}$ der geschätzten Standardabweichung der Residuen der logarithmierten Löhne der Männer zum Zeitpunkt $t$. Es gilt:

$$
\begin{aligned}
\hat{\theta}_{M i t} & =\frac{\ln y_{M i t}-\hat{\boldsymbol{\beta}}_{t}^{\prime} \boldsymbol{x}_{M i t}}{\hat{\sigma}_{t}}, \\
\hat{\theta}_{F i t} & =\frac{\ln y_{F i t}-\hat{\boldsymbol{\beta}}_{t}^{\prime} \boldsymbol{x}_{F i t}}{\hat{\sigma}_{t}} .
\end{aligned}
$$

Als Differenz der mittleren logarithmierten Löhne zum Zeitpunkt $t$ ergibt sich:

$$
\begin{aligned}
\overline{\ln y_{M t}}-\overline{\ln y_{F t}} & =\hat{\boldsymbol{\beta}}_{t}^{\prime}\left(\overline{\boldsymbol{x}}_{M t}-\overline{\boldsymbol{x}}_{F t}\right)+\hat{\sigma}_{t}\left(\overline{\hat{\theta}}_{M t}-\overline{\hat{\theta}}_{F t}\right) \\
\triangle \overline{\ln y_{t}} & =\hat{\boldsymbol{\beta}}_{t}^{\prime} \triangle \overline{\boldsymbol{x}}_{t}+\hat{\sigma}_{t} \triangle \overline{\hat{\theta}}_{t} .
\end{aligned}
$$

Der Term $\hat{\boldsymbol{\beta}}_{t}^{\prime}\left(\overline{\boldsymbol{x}}_{M t}-\overline{\boldsymbol{x}}_{F t}\right)$ entspricht dabei dem Teil der Lohnlücke, der auf unterschiedliche beoachtete Humankapitalausstattungen, der Term $\hat{\sigma}_{t}\left(\overline{\hat{\theta}}_{M t}-\overline{\hat{\theta}}_{F t}\right)$ dem Teil des Lohnunterschiedes, der auf Diskriminierung oder unterschiedliche unbeobachtete Charakteristika zurückzuführen ist. Die Standardabweichung $\hat{\sigma}_{t}$ kann dabei als (geschätzte) Ertragsrate dieser Fähigkeiten interpretiert werden. Da nur die Lohnstruktur der Männer $\hat{\boldsymbol{\beta}}_{M t}$ geschätzt wird, ergibt sich für $\overline{\hat{\theta}}_{M t}$ der Wert 0 , so dass $\triangle \overline{\hat{\theta}}_{t}=-\overline{\hat{\theta}}_{F t}$. Soll bei dynamischer Betrachtung der Unterschied zwischen den Zeitpunkten $t$ und $s$ mit $t>s$ bestimmt werden, so gilt:

$$
\begin{aligned}
\triangle \overline{\ln y_{t}}-\triangle \overline{\ln y_{s}} & =\underbrace{\hat{\boldsymbol{\beta}}_{s}^{\prime}\left(\triangle \overline{\boldsymbol{x}}_{t}-\triangle \overline{\boldsymbol{x}}_{s}\right)}_{\text {Ausstattungseffekt }}+\underbrace{\left(\hat{\boldsymbol{\beta}}_{t}-\hat{\boldsymbol{\beta}}_{s}\right)^{\prime} \triangle \overline{\boldsymbol{x}}_{t}}_{\text {beobachteter Preiseffekt }} \\
& +\underbrace{\left(\triangle \overline{\hat{\theta}}_{t}-\triangle \overline{\hat{\theta}}_{s}\right) \hat{\sigma}_{s}}_{\text {Lohnlückeneffekt }}+\underbrace{\triangle \overline{\hat{\theta}}_{t}\left(\hat{\sigma}_{t}-\hat{\sigma}_{s}\right)}_{\text {unbeobachteter Preiseffekt }} .
\end{aligned}
$$

Die Veränderung der Lohnlücke im Zeitverlauf kann nach Gleichung (3.42) in vier Komponenten zerlegt werden. Der Ausstattungseffekt ist der Beitrag, der auf Veränderungen in 
den Humankapitalausstattungen zurückzuführen ist. Wenn die relative Humankapitalausstattung der Frauen zunimmt und sich somit der Ausstattung der Männer anpasst, wird der Lohnunterschied vermindert. Der beobachtete Preiseffekt spiegelt die Veränderung in den Ertragsraten der beobachteten Humankapitalcharakteristika wider. Eine positive Veränderung bewirkt einen Anstieg der Lohndifferenz.

Der Lohnlückeneffekt erfasst Veränderungen der relativen Position von Männern und Frauen, d.h., ob Frauen höher oder niedriger in der männlichen Residualverteilung rangieren. Eine Verbesserung der Position der Frauen bewirkt einen negativen Lohnlückeneffekt. Ursache hierfür kann eine Annäherung der unbeobachteten Fähigkeiten von Männern und Frauen oder eine Reduzierung der Diskriminierung sein.

Der unbeobachtete Preiseffekt beschreibt, inwieweit eine Veränderung der Verteilung der männlichen Residuen im Zeitverlauf den Lohnunterschied beeinflusst unter der Annahme, dass Frauen dieselbe Position in der männlichen Residualverteilung beibehalten. Der Term $\left(\hat{\sigma}_{t}-\hat{\sigma}_{s}\right)$ kann dabei als Veränderung des Marktpreises für unbeobachtete Fähigkeiten angesehen werden, womit der unbeobachtete Preiseffekt analog dem beobachteten Preiseffekt für beobachtbare Humancharakteristika zu interpretieren ist. Juhn, Murphy und Pierce (1991) geben zu bedenken, dass die standardisierten Residuen nicht nur die unbeobachteten Fähigkeiten beschreiben, sondern auch Diskriminierung enthalten und zeigen, dass die Kosten der Diskriminierung gegenüber Frauen bei einem festen Wert für $d_{t}$ zunehmen, wenn die Marktpreise für unbeobachtete Fähigkeiten steigen. Im Folgenden sei $\theta_{i t}=\delta_{i t}+d_{i t}$, wobei $\delta_{i t}$ die unbeobachteten Fähigkeiten von Individuum $i$ relativ zum Durchschnitt und $d_{i t}$ Diskriminierung darstellen mit $d_{i t}=-d_{t}<0$, falls es sich bei Individuum $i$ um eine Frau und $d_{i t}=0$, falls es sich hierbei um einen Mann handelt. Gleichung (3.42) lautet in diesem Fall:

$$
\begin{aligned}
\triangle \overline{\ln y_{t}}-\triangle \overline{\ln y_{s}} & =\hat{\boldsymbol{\beta}}_{s}^{\prime}\left(\triangle \overline{\boldsymbol{x}}_{t}-\triangle \overline{\boldsymbol{x}}_{s}\right)+\left(\hat{\boldsymbol{\beta}}_{t}-\hat{\boldsymbol{\beta}}_{s}\right)^{\prime} \triangle \overline{\boldsymbol{x}}_{t} \\
& +\left(\triangle \overline{\hat{\theta}}_{t}-\triangle \overline{\hat{\theta}}_{s}\right) \hat{\sigma}_{s}+\left[\triangle \bar{\delta}_{t}\left(\hat{\sigma}_{t}-\hat{\sigma}_{s}\right)+d_{t}\left(\hat{\sigma}_{t}-\hat{\sigma}_{s}\right)\right] .
\end{aligned}
$$

Der erste Term in eckigen Klammern stellt erneut die Veränderung des Lohnunterschiedes dar, der auf unterschiedliche Fähigkeiten von Männern und Frauen zurückzuführen ist. Der zweite Term bringt dagegen zum Ausdruck, dass das Ausmaß der Lohnlücke bei steigenden Marktpreisen bei einem festen Wert für $d_{t}$ zunimmt.

Die Summe aus Lohnlücken- und unbeobachtetem Preiseffekt entspricht der Veränderung der unerklärten Lohnlücke, die im Allgemeinen als Diskriminierung angesehen wird.

\subsubsection{Empirische Resultate}

Für die Analyse der Lohnentwicklung im Zeitverlauf werden die Einkommen für 1985 und 1995, also vor und nach der Deutschen Wiedervereinigung, herangezogen und ent- 
sprechend des vom Statistischen Bundesamt erhobenen Verbraucherpreisindex 5 auf das Jahr 2006 umgerechnet, da sich die zuvor beschriebenen Methoden auf diesen Zeitpunkt bezogen. Die Stichprobe der Männer umfasst im Jahr 19852092 Beobachtungen und im Jahr 19952265 Beobachtungen, die der Frauen im Jahr 19851096 und im Jahr 19951918 Beobachtungen. Die Lohnentwicklung im Zeitraum von 1985-1995 ist in Tabelle (3.13) wiedergegeben. Der mittlere logatithmierte Lohn der Männer ist im Zeitraum von 1985-1995 um 0.187 Euro von 2.532 Euro auf 2.587 Euro angestiegen, der mittlere logarithmierte Lohn der Frauen um 0.253 Euro von 2.279 Euro auf 2.421 Euro, was einen Rückgang der Lohnlücke von 0.067 Euro bewirkt.

\begin{tabular}{cccc}
\hline \hline Jahr & $\begin{array}{c}\text { log. Lohn } \\
\text { (Euro) Männer }\end{array}$ & $\begin{array}{c}\text { log. Lohn } \\
\text { (Euro) Frauen }\end{array}$ & Differential \\
\hline \hline 1995 & 2.587 & 2.400 & 0.187 \\
1985 & 2.532 & 2.279 & 0.253 \\
\hline & & & -0.067 \\
\hline \hline
\end{tabular}

Tabelle 3.13: Entwicklung der Reallöhne im Zeitraum von 1985-1995

Bei der Auswahl der Lohnregressionsmodelle weist für das Jahr 1985 das Modell ohne die die Staatszugehörigkeit beschreibende Kovariable Ger, für das Jahr 1995 das Modell ohne die Kovariable Exp_2 den niedrigsten AIC auf. Für die weitere Studie wird das volle Modell mit sämtlichen Einflussvariablen herangezogen. Die Regressionsschätzungen der Ertragsraten für 1985 und 1995 sind in den Tabellen (3.14) und (3.15) abgebildet.

\begin{tabular}{lrrrrr}
\hline \hline & Estimate & Std.Error & t-value & $\operatorname{Pr}(>|\mathrm{t}|)$ & \\
\hline (Intercept) & 1.565 & 0.039 & 40.640 & 0.000 & $* * *$ \\
Edu & 0.045 & 0.003 & 14.266 & 0.000 & $* * *$ \\
Ten & 0.011 & 0.003 & 4.110 & 0.000 & $* * *$ \\
Ten_2 & -0.000 & 0.000 & -2.331 & 0.020 & $*$ \\
Exp & 0.026 & 0.003 & 9.953 & 0.000 & $* * *$ \\
Exp_2 & -0.001 & 0.000 & -8.117 & 0.000 & $* * *$ \\
Erljob & 0.054 & 0.014 & 3.771 & 0.000 & $* * *$ \\
Lt200 & 0.123 & 0.020 & 5.997 & 0.000 & $* * *$ \\
Lt2000 & 0.170 & 0.021 & 8.020 & 0.000 & $* * *$ \\
G2000 & 0.217 & 0.021 & 10.541 & 0.000 & $* * *$ \\
Ger & 0.005 & 0.015 & 0.308 & 0.758 & \\
\hline \hline
\end{tabular}

Tabelle 3.14: Koeffizientenschätzung für die Stichprobe der Männer 1985

Das Ergebnis der Zerlegung nach Juhn, Murphy und Pierce (1991) wird in Tabelle (3.16) wiedergegeben. Beobachteter und unbeobachteter Preiseffekt haben nur einen geringen Einfluss auf die Veränderung des Lohndifferentials. Hauptsächlich verantwortlich für den Rückgang des Lohndifferentials sind Ausstattungs- und Lohnlückeneffekt. Dies ist zum

\footnotetext{
${ }^{5}$ DESTATIS 2009, Verbraucherprisindex für Deutschland, Lange Reihe ab 1948-Juni 2009
} 


\begin{tabular}{lrrrrr}
\hline \hline & Estimate & Std.Error & t-value & $\operatorname{Pr}(>|\mathrm{t}|)$ & \\
\hline Edu & 0.040 & 0.004 & 10.695 & 0.000 & $* * *$ \\
Ten & 0.023 & 0.003 & 8.021 & 0.000 & $* * *$ \\
Ten_2 & -0.000 & 0.000 & -5.767 & 0.000 & $* * *$ \\
Exp & 0.006 & 0.003 & 2.196 & 0.028 & $*$ \\
Exp_2 & -0.000 & 0.000 & -1.259 & 0.208 & \\
Erljob & 0.086 & 0.017 & 5.186 & 0.000 & $* * *$ \\
Lt200 & 0.151 & 0.022 & 6.718 & 0.000 & $* * *$ \\
Lt2000 & 0.253 & 0.025 & 10.069 & 0.000 & $* * *$ \\
G2000 & 0.313 & 0.025 & 12.641 & 0.000 & $* * *$ \\
Ger & -0.096 & 0.026 & -3.747 & 0.000 & $* * *$ \\
\hline \hline
\end{tabular}

Tabelle 3.15: Koeffizientenschätzung für die Stichprobe der Männer 1995

\begin{tabular}{ccccc}
\hline \hline$\overline{\ln y_{t}}-\Delta \overline{\ln y_{s}}$ & $\begin{array}{c}\text { Ausstattungs- } \\
\text { effekt }\end{array}$ & $\begin{array}{c}\text { beobachteter } \\
\text { Preiseffekt }\end{array}$ & $\begin{array}{c}\text { Lohnlücken- } \\
\text { effekt }\end{array}$ & $\begin{array}{c}\text { unboebachteter } \\
\text { Preiseffekt }\end{array}$ \\
\hline-0.067 & -0.027 & 0.012 & -0.087 & 0.035 \\
\hline \hline
\end{tabular}

Tabelle 3.16: Ergebnisse der Dekomposition nach Juhn, Murphy und Pierce (1991)

einen darauf zurückzuführen, dass die relative Humankapitalausstattung der Frauen zunimmt und sich der Ausstattung der Männer annähert und zum anderen darauf, dass sich die unbeobachteten Fähigkeiten von Männern und Frauen anpassen und/oder Diskriminierung zurückgeht.

\subsection{Resüme}

In Deutschland liegt der durchschnittliche Lohn einer weiblichen Arbeitskraft unter dem einer männlichen Arbeitskraft. Diese Lohnunterschiede werden in politischen Debatten manchmal als Anzeichen dafür gewertet, dass viele Unternehmer Frauen diskriminieren. Allerdings ist zu beachten, dass selbst auf einem Arbeitsmarkt ohne Diskriminierung unterschiedliche Menschen Löhne in unterschiedlicher Höhe erhalten würden. Das alleinige Beobachten von Lohnunterschieden zwischen großen Gruppen erlaubt nach Mankiw (2001) keine Aussagen über die Gewichtigkeit der Diskriminierung. In der vorangegangenen Studie wurden unterschiedliche Verfahren zur Messung von Lohndiskriminierung vorgestellt und angewandt.

Die in Kapitel 3 beschriebenen Verfahren sind gut geeignet für die Dekomposition der Einkommensdifferenz der mittleren logarithmierten Löhne. Sie kommen zu dem Ergebnis, dass der weitaus größere Teil des Lohnunterschiedes auf Diskriminierung und nicht auf Unterschiede in den Humankapitalcharakteristika zurückzuführen ist. In der Praxis hat sich dabei die Mehode nach Neumark (1988) durchgesetzt. Hier wird zur Schätzung 
der Lohnstruktur in Abwesenheit von Diskriminierung sowohl die männliche als auch die weibliche Struktur herangezogen.

Die in Kapitel 4 vorgestellten Verfahren nach nach Brown, Moon und Zoloth (1980) sowie Appleton, Hoddinott und Krishnan (1999) berücksichtigen zusätzlich berufliche Segregation. Der Lohnunterschied lässt sich in einen Lohn- und einen Verteilungseffekt zerlegen. Auch hier ist der weitaus größere Teil der Lohndifferenz auf Diskriminierung zurückzuführen, wobei der Verteilungseffekt insgesamt eine nur untergeordnete Rolle spielt. Betrachtet man allerdings, wie sich Frauen und Männer ohne Diskriminierung auf die Berufsgruppen aufteilen würden, so muss das vorherige Ergebnis modifiziert werden. Weibliche Arbeitnehmer sind in einkommensstärkeren Berufen mit hohem Status unterpräsentiert, was für Diskriminierung gegenüber Frauen spricht. Allerdings ist der Anteil der Frauen in Berufen mit niedrigem Status ebenfalls zu gering, was für Diskriminierung gegenüber Männern sprechen würde.

Die Verfahren nach DiNardo, Fortin und Lemieux (1996) sowie Machado und Mata (2005), Melly (2005) ermöglichen die Dekomposition des unkorrigierten Lohndifferentials nicht nur für die mittleren Löhne, sondern für sämtliche Quantile der Lohnverteilung. Obwohl die beiden vorgeschlagenen Verfahren eine völlig unterschiedliche Herangehensweise bei der Schätzung der kontrafaktischen Lohnverteilung haben, ist das Ergebnis der Diskriminierungsmessung vergleichbar. Beide Methoden zeigen, dass bei den betrachteten Lohnquantilen der Einkommensunterschied größtenteils auf Diskriminierung zurückzuführen ist.

Das Ergebnis der Dekomposition nach Nopo (2006) kehrt bei einem gewählten Schwellenwert von null die Resultate der vorherigen Methoden um und kommt zu dem Ergebnis, dass Männer diskriminiert werden. Allerdings ist dieses Verfahren sehr kritisch zu bewerten. Bei einer Vielzahl von Merkmalen kann es schwierig sein, statistische Zwillinge zu finden. Die Güte einer auf der Bildung von Vergleichsgruppen basierenden Schätzung ist jedoch stets davon abhängig, inwieweit es gelingt, mit den beobachteten Merkmalen Vergleichbarkeit zwischen weiblichen und männlichen Vergleichspersonen herzustellen. Das oben beschriebene Matching-Verfahren stößt demzufolge dann an seine Grenzen, wenn eine Überlappung der Merkmale zwischen den zu vergleichenden Gruppen nicht oder in einem nur sehr geringen Maß gegeben ist. Wird der Schwellenwert gleich 70 gesetzt, werden jeder Frau sämtliche Männer als Vergleichspersonen zugeordnet mit der Folge, dass alle Arbeitskräfte dem common support zugehören. Die Terme $\triangle_{F}$ und $\triangle_{M}$ können nicht ermittlt werden. Da $\triangle_{x}$ gleich 0 und $\triangle_{O}$ dem Ausmaß der Lohnlücke entspricht, ist beim gewählten Schwellenwert die gesamte Einkommensdifferenz auf Diskriminierung zurückzuführen. Bei den ansonsten gewählten Toleranzbereichen sind die Ergebnisse der Dekomposition nach Nopo (2008) vergleichbar mit den zuvor beschriebenen Verfahren. Der weitaus größte Teil der Lonlücke ist auf Diskriminierung und nicht auf unterschiedliche Humankapitalausstattungen zurückzuführen. 
Mithilfe des Ansatzes nach Juhn, Murphy und Pierce (1991) wird die Lohnentwicklung im Zeitverlauf für 1985 und 1995, also vor und nach der Deutschen Wiedervereinigung, analysiert. Hauptsächlich verantwortlich für den Rückgang des Lohndifferentials sind Ausstattungs- und Lohnlückeneffekt. Dies ist zum einen darauf zurückzuführen, dass die relative Humankapitalausstattung der Frauen zunimmt und sich der Ausstattung der Männer annähert und zum anderen darauf, dass sich die unbeobachteten Fähigkeiten von Männern und Frauen anpassen und/oder Diskriminierung zurückgeht.

\section{Literatur}

Akaike, H. (1974) A new look at the statistical model identification, Automatic Control, IEEE Transactions on, Vol. 19 No. 6, S. 716 - 723 .

Appleton, S., Hoddinott, J., Krishnan, P. (1999). The Gender Wage Gap in Three African Countries, Economic Development and Cultural Change, Vol. 47, No. 2, S. 289-312.

Arrow, J. (1973). The Theory of Discrimination, in Ashenfelter, O.A. and Rees, A., Discrimination in Labor Markets, Princeton University Press.

Bayard, K., Hellerstein, J., Neumark, D., Troske, K. (2003). New Evidence on Sex Segregation and Sex Differences in Wages from Matched Employee-Employer Data, Journal of Labor Economics, Vol. 21, No. 4, S. 887-922.

Becker, G.-S. (1964, 1993, 3rd ed.). Human Capital: A Theoretical and Empirical Analysis with Special Reference to Education, Chicago, University of Chicago Press.

Becker, G.-S. (1957, 1971, 2nd ed.). The Economics of Discrimination, Chicago, University of Chicago Press.

Bergmann, B. (1974). Occupational Segregation, Wages and Profits when Employers Discriminate by Race or Sex, Eastern Economic Journal,Vol. 1, No. 2, S. 103-110.

Blau,F.-D., Kahn, L.-M. (2006). Wage Structure and Gender Earnings Differentials: An International Comparison, Economica, Vol. 63, No. 250, S. 29-62.

Blinder, A.-S. (1973). Wage Discrimination: Reduced Form and Structural Estimates, Journal of Human Resources, Vol. 8, No. 3, S. 436-455.

Brown, R.-S., Moon, M., Zoloth, B.-S. (1980). Incorporating Occupational Attainment in Studies of Male-Female Earnings Differentials, Journal of Human Resources, Vol. 15, No. 1, S. 3-28.

Cotton, J. (1988). On the Decomposition of Wage Differentials, The Review of Economics and Statistics, Vol. 70, No. 2, S. 236-243. 
Di Nardo, J., Fortin, N.-M., Lemieux, T. (1996). Labor Market Institutions and the Distribution of Wages, 1973-1992, A Semiparametric Approach Econometrica, Vol. 64, No. 5, S. 1001-1044.

Dolton, P.-J., KIDD, M.-P. (1994). Occupational access and wage discrimination, Oxford Bulletin of Economics and Statistics, Vol. 56, No. 4, S. 457-474.

Juhn, C., Murphy, K.-M., Pierce, B. (1991). Accounting for the Slowdown in BlackWhite Wage Convergence in Kosters, M., Workers and Their Wages, Washington DC.: AEI Press, S. $107-143$.

Mankiw, N.G. (2001). Grundzüge der Volkswirtschaftslehre Schäffer - Poeschel Verlag, Stuttgart, S. 444 f.

Machado, J.-A.-F., Mata, J. (2005). Counterfactual decomposition of changes in wage distributions using quantile regression, Journal of Applied Econometrics, Vol. 20, No 4, S. 445-465.

MacKinnon, J.-G., White, H. (1985). Some heteroskedasticity-consistent covariance matrix estimators with improved finite sample properties, Journal of Econometrics, Vol. 29, S. 305-325.

Mahalanobis, P.-C. (1936). On the generalized distance in statistics, Proc. Nat. Inst. Sci. India, Vol. 12, S. 49-55.

Melly, B. (2005). Public-private sector wage differentials in Germany: Evidence from quantile regression, Empirical Economics, Vol. 30, No 2, S. 505-520.

Miller, P.-W. (1987). The Wage Effect of the Occupational Segregation of Women in Britain, The Economic Journal, Vol. 97, S. 885-896.

Mincer, A.-M. (1974). Schooling, Experience, and Earnings, National Bureau of Economic Research, Inc..

Neumark, D. (1988). Employers' Discriminatory Behavior and the Estimation of Wage Discrimination, The Journal of Human Resources, Vol. 23, No. 3, S. 279-295.

Nopo, H. (2008). Matching as a Tool to Decompose Wage Gaps, The Review of Economics and Statistics, Vol. 90, No. 2, S. 290-299.

OAxACA, R.-L. (1973). Male-Female Wage Differentials in Urban Labor Markets, International Economic Review, Vol. 14, No. 3, S. 693-709.

OaxacA, R.-L., Ransom, M.-R. (1973). On discrimination and the decomposition of wage differentials, Journal of Econometrics, bf Volume 61, S. 5-21.

Phelps, E.-S. (1993). The Statistical Theory of Racism and Sexism, The American Economic Review, Vol. 62, No. 4, S. 659-661. 
Reimers, C.-W. (1983). Labour Market Discrimination Against Hispanic and Black Men, The Review of Economics and Statistics, Vol. 65, No. 4, S. 570-579.

Sorensen, E. (1990). The Crowding Hypothesis and Comparable Worth, Journal of Human Resources, Vol. 25, No. 1, S. 55-89.

Spence, A.-M. (1973). Job Market Signaling, The Quarterly Journal of Economics, Vol. 87, No. 3, S. 355-374.

Suen, W. (1997). Decomposing Wage Residuals: Unmeasured Skill or Statistical Artifact?, Journal of Labor Economics, Vol. 15, No. 3, S. 555-566.

Treiman, D. J. (1977). Occupational prestige in comparative perspective, Academic Press, New York. 